\title{
Seven amino acid types suffice to reconstruct the core fold of RNA polymerase
}

Authors: Sota Yagi ${ }^{1}$, Aditya K. Padhi ${ }^{1}$, Jelena Vucinic ${ }^{2,3}$, Sophie Barbe ${ }^{3}$, Thomas Schiex ${ }^{2}$, Reiko Nakagawa $^{1}$, David Simoncini ${ }^{4 *}$, Kam Y. J. Zhang ${ }^{1 *}$, Shunsuke Tagami ${ }^{*}$

\section{Affiliations:}

${ }^{1}$ RIKEN Center for Biosystems Dynamics Research, 1-7-22 Suehiro-cho, Tsurumi-ku, Yokohama, Kanagawa 230-0045, Japan

${ }^{2}$ Université Fédérale de Toulouse, ANITI, INRAE - UR 875, Toulouse, France

${ }^{3}$ TBI, Université Fédérale de Toulouse, CNRS, INRAE, INSA, ANITI, Toulouse, France

${ }^{4}$ Université Fédérale de Toulouse, ANITI, IRIT - UMR 5505, Toulouse, France.

*Corresponding Authors. D.S. (david.simoncini@gmail.com), K.Y.J.Z. (kamzhang@riken.jp),

S.T. (shunsuke.tagami@riken.jp) 
Abstract: The extant complex proteins must have evolved from ancient short and simple ancestors. Nevertheless, how such prototype proteins emerged on the primitive earth remains enigmatic. The double-psi beta-barrel (DPBB) is one of the oldest protein folds and conserved in various fundamental enzymes, such as the core domain of RNA polymerase. Here, by reverse engineering a modern DPBB domain, we reconstructed its evolutionary pathway started by “interlacing homodimerization" of a half-size peptide, followed by gene duplication and fusion. Furthermore, by simplifying the amino acid repertoire of the peptide, we successfully created the DPBB fold with only seven amino acid types (Ala, Asp, Glu, Gly, Lys, Arg, and Val), which can be coded by only GNN and ARR $(\mathrm{R}=\mathrm{A}$ or $\mathrm{G})$ codons in the modern translation system. Thus, the DPBB fold could have been materialized by the early translation system and genetic code. 
Modern proteins with large and complex structures are generally thought to have evolved from small and simple ancient proteins with "prototype folds" (e.g., Rossmann fold, ferredoxin fold, and $(\beta / \alpha)_{8}$-barrel) $(1-10)$. These prototype folds must have played essential roles in the early evolution of life, as they are often conserved in fundamental biochemical pathways such as metabolism, replication, transcription, and translation (11-14). However, it remains elusive how such prototype folds emerged on the ancient earth, where the primitive translation system likely performed imprecise syntheses of short peptides composed of fewer amino acids as compared to modern proteins (15-17). Especially, the components of the earliest genetic code are still an open question, as the 9-13 amino acid types used in previous ancestral protein reconstructions are at scattered positions in the modern codon table (18-21).

The double-psi beta-barrel (DPBB) has one of the most important functions and complicated structures among such prototype folds. It is conserved in several enzymes in fundamental biochemical processes (Fig. S1) (13, 22-24). For example, the formate dehydrogenase in the oldest carbon fixation system, the Wood-Ljungdahl pathway, possesses a DPBB domain (25). The DPBB and a few related small $\beta$-barrel folds are also often conserved in essential proteins from transcription and translation systems $(26,27)$. Most notably, the active site of RNA polymerase from all cellular life is composed of two DPBB folds, and thus the original core of RNA polymerase may have emerged by the duplication of an ancestral DPBB $(14,28-30)$.

The DPBB fold is a six-stranded $\beta$-barrel consisting of two pseudo-symmetric $\beta \beta \alpha \beta$ units with detectable structural and sequence homologies (Fig. 1A). All six $\beta$-strands are aligned in an interdigitated manner, giving its pseudoknot-like topology. The loop connecting $\beta 1$ and $\beta 2$ ( $\beta 1^{\prime}$ and $\beta 2^{\prime}$ ) crosses the loop between $\beta 2^{\prime}$ and $\beta 3^{\prime}$ ( $\beta 2$ and $\beta 3$ ), thus generating a shape similar to the Greek letter $\psi$. Although some other prototype folds with similar pseudo-symmetries apparently originated by oligomerization and gene duplication of shorter peptides (31), it remains uncertain 
if the interdigitating fold of DPBB could have formed in such a simple oligomerization process $(13,22,27,29)$. So far, no modern DPBB structure composed of a perfect sequence repeat or a dimer of shorter peptides has been reported, and thus the origin of the DPBB fold remains elusive.

In this study, we demonstrate that the DPBB fold could have emerged by oligomerization and gene-duplication of a shorter and simpler peptide, by reconstructing the DPBB domains comprising perfect sequence repeats and then self-dimerizing peptides. Surprisingly, even chemically synthesized peptides could fold into the complicated pseudoknot-like topology. We also eliminated several amino acid types from the designs, and confirmed that only seven amino acid types (Ala, Asp, Glu, Gly, Lys, Arg, and Val) are sufficient for the DPBB fold. These amino acid types can be coded by only GNN and ARR ( $\mathrm{R}=\mathrm{A}$ or $\mathrm{G})$ codons in the modern translation system. These results reveal the plausible ancient pathway for the emergence of the complicated prototype fold and transcription machinery, which coevolved with the early translation system and genetic code.

\section{Reconstruction of DPBB domains with perfect sequence symmetry}

We first searched for extant DPBB domains with high internal sequence homologies, as the starting models to reconstruct a DPBB domain with perfect sequence repeats. The DPBB domain of a molecular chaperone, valosin-containing protein (VCP) from Thermoplasma acidophilum (taVCP_DPBB), reportedly has relatively high sequence identity (37\%) between its N- and Cterminal halves (Table S1, Fig. S1F) (22). We determined its crystal structure in the isolated form, to confirm that it adopts the DPBB fold even without the other domains of VCP (Fig. 1B, Table S2). taVCP_DPBB also shares high structural homology with the core domain of RNA polymerase $(\mathrm{RMSD}=1.6 \AA)$, indicating their common origin (Fig. S1G). 
Using the sequence of taVCP_DPBB as a query, we searched for VCP_DPBB domains with higher internal sequence homology from other organisms and found that the VCP_DPBBs from Methanopyrus kandleri (mkVCP_DPBB) and Aeropyrum pernix (apVCP_DPBB) have $42 \%$ and $45 \%$ internal sequence identities, respectively (Tables S1, S3). We determined their crystal structures (Fig. 1C and D, Table S2) and confirmed that they also exhibit more precise structural symmetries than taVCP_DPBB (Fig. S2). Thus, these two VCP_DPBBs were estimated to have similar sequences and structures to the ancestral DPBB with perfect sequence repeats. Furthermore, mkVCP_DPBB and apVCP_DPBB were from extreme thermophiles and showed high thermostability $\left(T_{m}>69^{\circ} \mathrm{C}\right)$ and refolding ability (Fig. S3 and Table S4). The coexistence of the ancestral symmetric feature and the extreme thermostability in these DBPP domains is also consistent with the widely supported hypothesis suggesting that the last universal common ancestor (LUCA) was a hyper-thermophilic organism $(32,33)$.

We then reconstructed DPBB domains with perfect internal repeats, by a method based on symmetrically-conserved positions (SC-design, Supplementary Text, Figs. S4-6, Tables S1, S2, S5). We engineered mkVCP_DPBB and apVCP_DPBB by replacing the residues in their nonsymmetric positions with the symmetrically-conserved residues in VCP_DPBBs from different organisms. A few cycles of mutagenesis and structural confirmation by circular dichroism (CD), SEC, and X-ray crystallography finally resulted in two mutants, mkDPBB_sym_86 and apDPBB_sym_84, with $86 \%$ and $84 \%$ internal sequence identities, respectively (Fig. 1E and Table S1). These designs only have a limited number of non-symmetric positions clustered in two areas in their tertiary structures, defined as "cluster-1 and -2" in Fig. 1E (also see Fig. S7). By grafting the amino acid residues from each of the two clusters to the other one, we then designed four DPBBs with perfect internal sequence identities (mkDPBB_sym1, mkDPBB_sym2, apDPBB_sym1, and apDPBB_sym2, Table S1). Although one of them (apDPBB_sym2) could not 
be purified due to its poor stability, the other three were readily purified. They eluted as monomeric proteins in SEC analyses, exhibited similar $\alpha / \beta$ CD spectra with the native DPBB proteins, and retained high thermostability $\left(T_{m} \geq 85^{\circ} \mathrm{C}\right.$, Fig. S5, Table S5). Finally, we solved the crystal structures of mkDPBB_sym1 and mkDPBB_sym2 (Fig. 1F, Table S2), which have almost perfect structural symmetries. These results strongly indicate that the DPBB fold originally arose from a perfectly symmetric ancestor.

\section{Computational designs and possible sequence diversity of symmetric DPBBs}

We also implemented two computational approaches for symmetric designs of DPBB to test if diverse strategies could result in different/similar sequences. The first computational approach was a modified "reverse engineering evolution" (34) (RE-design, Supplementary Text, Fig. 2A, Fig. S8). In this methodology, we constructed a phylogenetic tree with the respective aligned sequences, which were subsequently used as input to generate the ancestral sequences. The predicted ancestral sequences were mapped onto a manually constructed, perfectly symmetrical mkVCP structural backbone model, and were evaluated by their Rosetta energy scores (Fig. S9). The seven topscoring designs were chosen for the experimental evaluation (Table S1).

The second computational approach was a multi-state computational protein design (MS-design, Methods, Fig. 2B, Fig. S10). This method describes the target protein structure as an ensemble of fixed backbone conformational states, to account for protein flexibility (35). The sequence that minimizes the average energy over all conformational states is computed by the AI automated reasoning prover ToulBar2 (36). The MS-designs were less homologous to the other designs since they were obtained by energy minimization, without any knowledge extraction from other homologous DPBB domains. From the top-scoring designs, we chose the two with the highest 
sequence dissimilarities from the SC- or RE-designs for the experimental evaluation (Fig. S11, Table S1).

We tried to express the seven RE-designs and two MS-designs in E. coli. Although two REdesigns were not expressed well, the other designs were readily purified, exhibited similar properties to mkVCP_DPBB in SEC and CD analyses, and retained high thermostability $\left(T_{m} \geq 67\right.$ ${ }^{\circ}$ C, Figs. S12 and S13, Table S5). Furthermore, we solved the crystal structures of three REdesigns and one MS-design and confirmed that they adopt the designed structures (Fig. 2D, Fig. S6B-E, Table S2).

Interestingly, while all ten purified designs (three SC-designs and seven computational designs) have almost identical properties (structure, thermostability), their sequences are very diverse (Fig. $2 \mathrm{C}$ and Fig. S11, Supplementary Text). Only 34 positions ( 17 positions $\times 2$ repeats) were perfectly conserved in the $\sim 90$ a.a. designs (Fig. 2D). The high sequence diversity and success ratios of these designs (Table 1) indicate that the symmetric DPBB fold can be adopted by a significant variety of sequences and had a high probability of emerging during the early evolution of life.

\section{Homo-dimerization of halved fragments}

To further investigate if the DPBB structure can be formed by the homo-dimerization of halved fragments ( 46 aa) of symmetric DPBBs, we expressed the N-terminal halves of the four SCdesigns (mk1h, mk2h, ap1h, and ap2h; Fig. 3A and 3B and Table S1). All four fragments were expressed as soluble peptides in E. coli and formed dimers with $\alpha / \beta$ structures (Fig. S14 and Table S6). Crystallographic analyses of $\mathrm{mk} 2 \mathrm{~h}$ and ap $1 \mathrm{~h}$ demonstrated that the halved fragments adopt the DPBB fold by interlacing homo-dimerization (Fig. 3C, Fig. S6F and Table S2). They also exhibited high thermostability and refoldability after heat denaturation (Fig. 3D, 3E, Fig. S14 and 
Table S6). These results strongly indicate that the DPBB fold originally emerged simply by the homo-dimerization of a short peptide, in spite of its complicated interlaced topology.

We also tested the foldability of the chemically-synthesized $\mathrm{mk} 2 \mathrm{~h}$ peptide. First, the dried powder of $\mathrm{mk} 2 \mathrm{~h}(95.14 \%$ purity) was dissolved in $20 \mathrm{mM}$ Bis-tris $\mathrm{HCl}, \mathrm{pH} 6.0$, with $150 \mathrm{mM}$ $\mathrm{NaCl}$, and subjected to crystallization screening. We readily obtained some crystals under various conditions (Table S7) and determined its structure to confirm that it also adopts the DPBB structure (Fig. 3C, Table S2). Thus, the chemically-synthesized peptide can fold precisely into the interlacing DPBB structure without any factors/environment in the cell, demonstrating that the amino acid sequence of $\mathrm{mk} 2 \mathrm{~h}$ encodes its homo-dimerizing and folding information.

Next, to investigate whether the peptide could fold even in the presence of contaminants with similar sequences, we analyzed the foldability of a low-purity sample of mk $2 \mathrm{~h}$ containing byproducts from the chemical synthesis $(71.16 \%$ purity). In the SEC analysis, two major peaks corresponding to aggregated and dimer species appeared (Fig. 3F). The dimer fraction showed the typical CD spectra for $\alpha / \beta$ proteins, while the aggregated fraction exhibited a disordered conformation (Fig. 3G). The LC/MS analysis revealed that most of the contaminants were enriched in the aggregated fraction (e.g., the 4748.6 Da byproduct corresponding to a serine deletion, Fig. $3 \mathrm{H}$ and Table S8). In contrast, the full-length peptide (4835.6 Da) was enriched in the dimer fraction. This auto-purification phenomenon during protein folding was also observed with another design, mk1h (Fig. S15 and Table S8). Therefore, the homo-dimerization and folding processes of the peptides likely worked as a purification/selection system by excluding contaminated sequences, and thus might have enabled the production of the DPBB domains by an imprecise ancient translation system or prebiotic peptide synthesis.

\section{Reduction of amino acid repertory}


Interestingly, mk2h contained only 13 amino acid types, although we did not intend to enrich/exclude any specific amino acid species in the engineering process (Fig. 4A, Table S9). To examine how many and what kind of amino acid species are required to comprise a DPBB scaffold, we tried to further simplify the amino acid repertoire of mk2h (Fig. 4B). In mk2h, Ile, Leu, Met, Pro, and Ser were used only once or twice. Tyrosine was the only aromatic amino acid type and used just three times (Fig. 4A). Thus, we replaced each of these amino acid types with other amino acid residues conserved in different organisms or possessing similar chemical/structural properties (e.g., Ile to Val) to generate mutants containing 12 amino acid repertoires (Table S1). MD simulations estimated that none of the mutations would have a devastating effect on the domain structure (Fig. S16). All mutants were eluted as homo-dimers in SEC and their CD spectra were similar to that of mk2h (Fig. S17), although some of them exhibited lower $T_{m}$ values than $\mathrm{mk} 2 \mathrm{~h}$ (Table S10). Furthermore, the crystal structures of $m k 2 h \_\Delta \mathrm{P}$ and $\mathrm{mk} 2 \mathrm{~h} \_\Delta \mathrm{Y}$ revealed they indeed adopt the DPBB fold (Fig. S6G and H, Table S2). Therefore, these amino acid species may have contributed to the thermostability of $\mathrm{mk} 2 \mathrm{~h}$, but are not essential to form the DPBB fold.

Subsequently, we created a mutant in which Met, Ile, and Leu were eliminated simultaneously (mk2h_AMIL). The mutant was expressed as a homo-dimer and showed typical CD spectra for $\alpha / \beta$ proteins $\left(T_{m}=54.6^{\circ} \mathrm{C}\right.$, Fig. S18A and Table S10). The X-ray crystallographic analysis of mk2h_dMIL revealed it has a very primitive hydrophobic core composed of only Val and Ala, with some unfilled cavities (Fig. 4C). Recently, a de novo designed protein with a hydrophobic core composed mostly of Valine residues was reported, indicating that the backbone structure is the main contributor to its thermostability (37). GD-box, a structural motif conserved among DPBB and various protein folds, has also been suggested to stabilize the protein tertiary structures by tethering noncontiguous segments with hydrogen bonds between the main chains (38). Such a stable backbone of the DPBB fold would have allowed the emergence of a globular protein with 
only simple and small hydrophobic amino acids during early protein evolution. Unlike hydrophilic interactions, hydrophobic interactions do not require precise angles or directions, and thus such simple hydrophobic cores might emerge relatively easily in aqueous environments without optimizing the sizes or compositions of their amino acid residues.

We also tried to exclude two amino acid types (Pro/Ser, Pro/Tyr, or Ser/Tyr) in mk2h_ $\Delta \mathrm{MIL}$

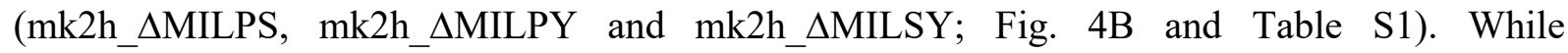
$\mathrm{mk} 2 \mathrm{~h} \_\Delta \mathrm{MILPY}$ and $\mathrm{mk} 2 \mathrm{~h} \_\Delta \mathrm{MILSY}$ were eluted as unfolded aggregates in SEC, mk2h_AMILPS eluted as a dimer and its CD spectra were similar to those of mk2h (Fig. S18B-D and Table S10). The crystal structures of E. coli-produced and chemically-synthesized mk2h_AMILPS were also determined (Fig. S6I and J, Table S2). Although mk2h__MILPS remained thermostable $\left(T_{m}=\right.$ $50.5^{\circ} \mathrm{C}$ ), refoldability was not observed (Fig. S18B).

Finally, we designed an mk2h variant, mk2h_AMILPYS, with only seven amino acid types (Ala, Asp, Glu, Gly, Lys, Arg, and Val) by combining the mutations in mk2h_AMILPS and mk2h_ $\Delta \mathrm{Y}$ (Fig. 4B). Although the peptides exhibited unfolded properties in the SEC and CD analyses (Fig. S18E), we obtained crystals from two conditions containing the chemicallysynthesized peptide (Fig. S19). The crystal structures demonstrated that mk2h__MILPYS adopts the DPBB structure through homo-dimerization (Fig. 4D, Fig. S6K, and Table S2). In the structures, the positively-charged pocket conserved in the extant VCP_DPBB is occupied by the fundamental metabolites, malonate or malic acid, contained in the crystallization conditions (Fig. 4D, Fig. S20), which might also have assisted in the folding of mk2h_AMILPYS. Thus, despite its limited folding propensity, the 43 a.a. peptide with only seven amino acid types can homo-dimerize and fold into the DPBB structure. Additional amino acids would have been incorporated for higher stability and foldability during protein evolution (Fig. 4B). 


\section{Discussion}

In this study, we demonstrated that one of the prototypic protein folds, DPBB, can be reconstructed by perfect sequence repeats (Figs. 1,2), as well as by dimers of the halved fragments (Fig. 3), indicating that DPBB originally emerged by the self-dimerization of $\sim 40$ a.a. peptides and then evolved via gene duplication and fusion. Chemically synthesized peptides were able to fold into the complicated interlaced topology, without any support from the modern biological machinery, and still retained thermostability and refoldability (Fig. 3). Furthermore, the success rate and diversity of our designs were surprisingly high (Fig. 2C and Fig. S11, Table 1). The fragmented peptides also exhibited auto-purification ability (Fig. $3 \mathrm{H}$ ) and high amenability to engineering (Fig. 4B). Thus, the DPBB fold probably had a significant chance of emerging from the pool of primitive peptides synthesized by an immature/imprecise translation system on the early earth. Nature can find and utilize such a complicated protein fold as long as it is stable enough, even though in theory it appears unrealistic to the human eye.

By simplifying mk2h, we further constructed the DPBB fold with only seven amino acid types, Ala, Asp, Glu, Gly, Lys, Arg, and Val (mk2h__MILPYS, Fig. 4). The previously reconstructed ancestral proteins with $\sim 10$ amino acid types also contain most of them $(19-21,39)$, indicating that they were shared by various prototype proteins. Interestingly, these seven amino acid types can be coded on a clearly defined area in the standard codon table (GNN and ARR) (Fig. 4E). The five amino acids coded by GNN (Ala, Asp, Glu, Gly, and Val) were probably adopted into the earliest genetic code because they can be easily produced in the prebiotic environment $(16,17$, 40-42). The other two amino acids (Arg and Lys) have cationic side chains and tend to interact with nucleic acid polymers. Arginine could have been abiotically synthesized by sharing some precursors to nucleotides (42). Recent studies have shown that peptide analogs enriched with such basic residues can be prebiotically synthesized and mutually stabilize RNA $(43,44)$. Simple 
peptides containing lysine residues could also enhance the activities of ribozymes (45). Considering the fact that the genetic code is realized by RNA-based machinery, arginine and lysine were probably readily recruited into the early genetic code during evolution. This idea is also supported by the fact that the core domain of RNA polymerase, the enzyme responsible for the synthesis of rRNA, mRNA, and tRNA, is composed of the DPBB fold. Like its modern descendants, the ancient symmetric DPBB fold might have interacted with nucleotide-related molecules, as the conserved positively-charged pockets on some symmetrized or simplified designs are occupied by negatively-charged ligands in their crystal structures (Fig. S20). Thus, the DPBB fold was likely established at an early evolutionary stage of the genetic code and supported the ancient RNA-based biosystem when only $\sim 7$ amino acid types were available.

\section{References}

1. R. V. Eck, M. O. Dayhoff, Evolution of the structure of ferredoxin based on living relics of primitive amino acid sequences. Science (80-. ). 152, 363-366 (1966).

2. J. Söding, A. N. Lupas, More than the sum of their parts: On the evolution of proteins from peptides. BioEssays. 25, 837-846 (2003).

3. M. L. Romero Romero, A. Rabin, D. S. Tawfik, Functional Proteins from Short Peptides: Dayhoff's Hypothesis Turns 50. Angew. Chemie - Int. Ed. 55, 15966-15971 (2016).

4. S. Setiyaputra, J. P. MacKay, W. M. Patrick, The structure of a truncated phosphoribosylanthranilate isomerase suggests a unified model for evolution of the $(\beta \alpha) 8$ barrel fold. J. Mol. Biol. 408, 291-303 (2011).

5. D. Lang, R. Thoma, M. Henn-Sax, R. Sterner, M. Wilmanns, Structural evidence for evolution of the $\beta / \alpha$ barrel scaffold by gene duplication and fusion. Science (80-. ). 289, $1546-1550(2000)$.

6. J. M. Thornton, C. A. Orengo, A. E. Todd, F. M. G. Pearl, Protein folds, functions and evolution. J. Mol. Biol. 293, 333-342 (1999).

7. V. Alva, J. Söding, A. N. Lupas, A vocabulary of ancient peptides at the origin of folded proteins. Elife. 4, 1-19 (2015).

30 8. B. G. Ma, L. Chen, H. F. Ji, Z. H. Chen, F. R. Yang, L. Wang, G. Qu, Y. Y. Jiang, C. Ji, H. Y. Zhang, Characters of very ancient proteins. Biochem. Biophys. Res. Commun. 366, 607-611 (2008).

9. M. Henn-Sax, B. Höcker, M. Wilmanns, R. Sterner, Divergent evolution of $(\beta \alpha) 8$-barrel enzymes. Biol. Chem. 382, 1315-1320 (2001).

10. B. Höcker, Design of proteins from smaller fragments-learning from evolution. Curr. Opin. Struct. Biol. 27, 56-62 (2014). 
11. H. Raanan, S. Poudel, D. H. Pike, V. Nanda, P. G. Falkowski, Small protein folds at the root of an ancient metabolic network. Proc. Natl. Acad. Sci. U. S. A. 117, 7193-7199 (2020).

12. A. D. Goldman, R. Samudrala, J. A. Baross, The evolution and functional repertoire of translation proteins following the origin of life. Biol. Direct. 5 (2010), doi:10.1186/17456150-5-15.

13. R. M. Castillo, K. Mizuguchi, V. Dhanaraj, A. Albert, T. L. Blundell, A. G. Murzin, A six-stranded double-psi $\beta$ barrel is shared by several protein superfamilies. Structure. 7, 227-236 (1999).

14. E. V. Koonin, M. Krupovic, S. Ishino, Y. Ishino, The replication machinery of LUCA: Common origin of DNA replication and transcription. BMC Biol. 18, 1-8 (2020).

15. E. V. Koonin, A. S. Novozhilov, Origin and evolution of the genetic code: The universal enigma. IUBMB Life. 61, 99-111 (2009).

16. K. Ikehara, Y. Omori, R. Arai, A. Hirose, A novel theory on the origin of the genetic code: A GNC-SNS hypothesis. J. Mol. Evol. 54, 530-538 (2002).

17. K. Macé, R. Gillet, Origins of tmRNA: The missing link in the birth of protein synthesis? Nucleic Acids Res. 44, 8041-8051 (2016).

18. K. U. Walter, K. Vamvaca, D. Hilvert, An active enzyme constructed from a 9-amino acid alphabet. J. Biol. Chem. 280, 37742-37746 (2005).

19. L. M. Longo, J. Lee, M. Blaber, Simplified protein design biased for prebiotic amino acids yields a foldable, halophilic protein. Proc. Natl. Acad. Sci. U. S. A. 110, 2135-2139 (2013).

20. R. Shibue, T. Sasamoto, M. Shimada, B. Zhang, A. Yamagishi, S. Akanuma, Comprehensive reduction of amino acid set in a protein suggests the importance of prebiotic amino acids for stable proteins. Sci. Rep. 8, 1-8 (2018).

21. M. Kimura, S. Akanuma, Reconstruction and Characterization of Thermally Stable and Catalytically Active Proteins Comprising an Alphabet of $\sim 13$ Amino Acids. J. Mol. Evol. 88, 372-381 (2020).

22. M. Coles, T. Diercks, J. Liermann, A. Gröger, B. Rockel, W. Baumeister, K. K. Koretke, A. Lupas, J. Peters, H. Kessler, The solution structure of VAT-N reveals a "missing link" in the evolution of complex enzymes from a simple $\beta \alpha \beta \beta$ element. Curr. Biol. 9, $1158-$ 1168 (1999).

23. O. Erbilgin, M. Sutter, C. A. Kerfeld, The Structural Basis of Coenzyme A Recycling in a Bacterial Organelle. PLoS Biol. 14, 1-20 (2016).

24. Y. Nishitani, R. Aono, A. Nakamura, T. Sato, H. Atomi, T. Imanaka, K. Miki, Structure analysis of archaeal AMP phosphorylase reveals two unique modes of dimerization. $J$. Mol. Biol. 425, 2709-2721 (2013).

25. D. Niks, R. Hille, Molybdenum- and tungsten-containing formate dehydrogenases and formylmethanofuran dehydrogenases: Structure, mechanism, and cofactor insertion. Protein Sci. 28, 111-122 (2019).

26. P. Youkharibache, S. Veretnik, Q. Li, K. A. Stanek, C. Mura, P. E. Bourne, The Small $\beta$ Barrel Domain: A Survey-Based Structural Analysis. Structure. 27, 6-26 (2019).

27. V. Alva, K. K. Koretke, M. Coles, A. N. Lupas, Cradle-loop barrels and the concept of metafolds in protein classification by natural descent. Curr. Opin. Struct. Biol. 18, 358365 (2008).

28. L. Sauguet, The Extended "Two-Barrel” Polymerases Superfamily: Structure, Function and Evolution. J. Mol. Biol. 431, 4167-4183 (2019).

29. Z. F. Burton, The old and new testaments of gene regulation: Evolution of multi-subunit RNA polymerases and co-evolution of eukaryote complexity with the RNAP II CTD. Transcription. 5 (2014), doi:10.4161/trns.28674.

30. T. Fouqueau, F. Blombach, F. Werner, Evolutionary Origins of Two-Barrel RNA Polymerases and Site-Specific Transcription Initiation. Annu. Rev. Microbiol. 71, 331-348 (2017). 
31. V. Alva, A. N. Lupas, From ancestral peptides to designed proteins. Curr. Opin. Struct. Biol. 48, 103-109 (2018).

32. A. Nasir, K. M. Kim, G. Caetano-Anollés, A Phylogenomic Census of Molecular Functions Identifies Modern Thermophilic Archaea as the Most Ancient Form of Cellular Life. Archaea. 2014 (2014), doi:10.1155/2014/706468.

33. S. Akanuma, Y. Nakajima, S. I. Yokobori, M. Kimura, N. Nemoto, T. Mase, K. I. Miyazono, M. Tanokura, A. Yamagishi, Experimental evidence for the thermophilicity of ancestral life. Proc. Natl. Acad. Sci. U. S. A. 110, 11067-11072 (2013).

34. A. R. D. Voet, H. Noguchi, C. Addy, D. Simoncini, D. Terada, S. Unzai, S. Y. Park, K. Y. J. Zhang, J. R. H. Tame, Computational design of a self-assembling symmetrical $\beta$ propeller protein. Proc. Natl. Acad. Sci. U. S. A. 111, 15102-15107 (2014).

35. J. Vucinic, D. Simoncini, M. Ruffini, S. Barbe, T. Schiex, Positive multistate protein design. Bioinformatics. 36, 122-130 (2020).

36. M. C. Cooper, S. de Givry, M. Sanchez, T. Schiex, M. Zytnicki, T. Werner, Soft arc consistency revisited. Artif. Intell. 174, 449-478 (2010).

37. R. Koga, M. Yamamoto, T. Kosugi, N. Kobayashi, T. Sugiki, T. Fujiwara, N. Koga, Robust folding of a de novo designed ideal protein even with most of the core mutated to valine. Proc. Natl. Acad. Sci. U. S. A. 117, 31149-31156 (2020).

38. V. Alva, S. Dunin-Horkawicz, M. Habeck, M. Coles, A. N. Lupas, The GD box: A widespread noncontiguous supersecondary structural element. Protein Sci. 18, 1961-1966 (2009).

39. L. M. Longo, D. Despotović, O. Weil-Ktorza, M. J. Walker, J. Jabłońska, Y. FridmannSirkis, G. Varani, N. Metanis, D. S. Tawfik, Primordial emergence of a nucleic acidbinding protein via phase separation and statistical ornithine-to-arginine conversion. Proc. Natl. Acad. Sci. U. S. A. 117, 15731-15739 (2020).

40. S. L. Miller, A production of amino acids under possible primitive earth conditions. Science (80-. ). 117, 528-529 (1953).

41. A. P. Johnson, H. J. Cleaves, J. P. Dworkin, D. P. Glavin, A. Lazcano, J. L. Bada, The Miller volcanic spark discharge experiment. Science (80-. ). 322, 404 (2008).

42. B. H. Patel, C. Percivalle, D. J. Ritson, C. D. Duffy, J. D. Sutherland, Common origins of RNA, protein and lipid precursors in a cyanosulfidic protometabolism. Nat. Chem. 7, 301307 (2015).

43. M. Frenkel-Pinter, J. W. Haynes, A. M. Mohyeldin, C. Martin, A. B. Sargon, A. S. Petrov, R. Krishnamurthy, N. V. Hud, L. D. Williams, L. J. Leman, Mutually stabilizing interactions between proto-peptides and RNA. Nat. Commun. 11, 1-14 (2020).

44. M. Frenkel-Pinter, J. W. Haynes, C. Martin, A. S. Petrov, B. T. Burcar, R. Krishnamurthy, N. V. Hud, L. J. Leman, L. D. Williams, Selective incorporation of proteinaceous over nonproteinaceous cationic amino acids in model prebiotic oligomerization reactions. Proc. Natl. Acad. Sci. U. S. A. 116, 16338-16346 (2019).

45. S. Tagami, J. Attwater, P. Holliger, Simple peptides derived from the ribosomal core potentiate RNA polymerase ribozyme function. Nat. Chem. 9, 325-332 (2017).

\section{Acknowledgements}

This work is based on experiments performed at KEK (project number: 20G056) and SPring-

8. The authors are grateful to the beamline staff scientists at KEK, SPring-8, and SLS. We thank

Hideaki Niwa, Toshiaki Hosaka, and Kentaro Ihara for helping with the X-ray diffraction experiments. We also thank Shigehiro Kuraku for assistance in the LC-MS analysis. We are deeply 
grateful to Ryutaro Furukawa for providing an informative in-house protein database to search for natural DPBB proteins. We acknowledge RIKEN ACCC for the supercomputing resources at the Hokusai BigWaterfall supercomputer used in this study. A.K.P. acknowledges the Japan Society for the Promotion of Science (JSPS), Govt. of Japan, for the research fellowship. S.Y., K.Y.J.Z. and S.T. were supported by JSPS (20K15854, 18H02395 and 18H01328). This work was also supported by the French ANR through an ANR-19-PI3A-0004 grant. We thank the CALMIP HPC center for computational resources. We thank Satoshi Akanuma, Hiroshi Sasaki, Loren D. Williams, and Claudia Alvarez-Carreno for fruitful discussions. 

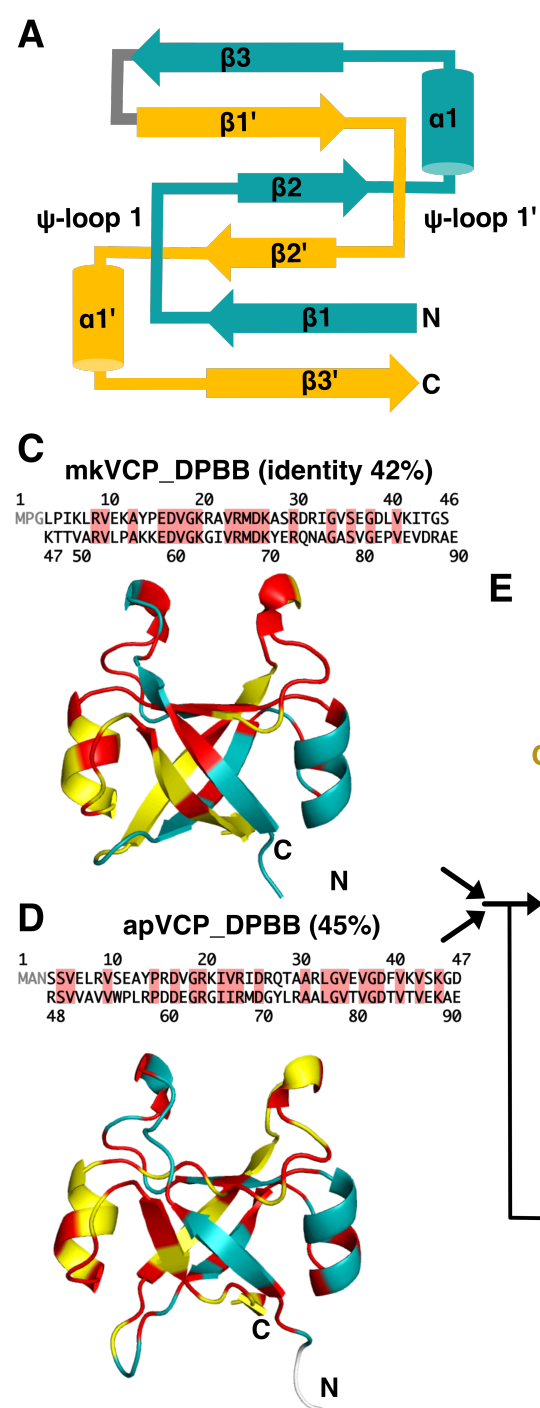

Figure 1. Structures and symmetrical engineering of the DPBB domains. (A) Topology

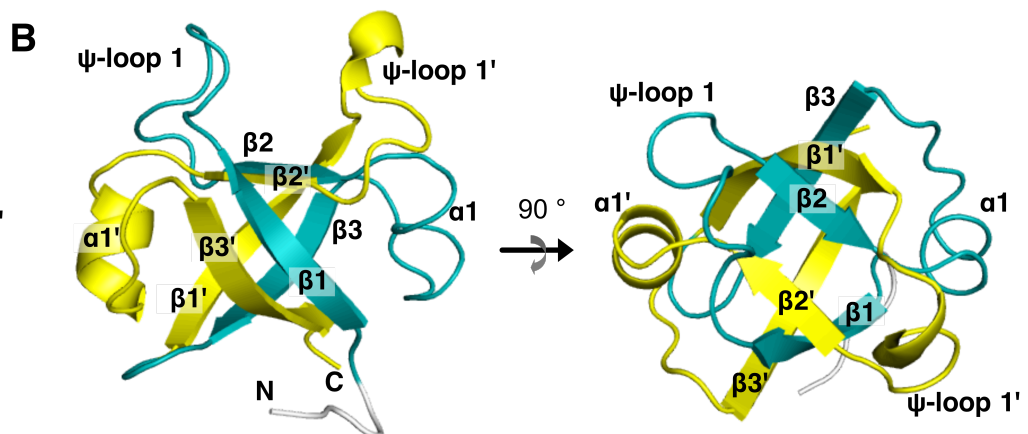

$\mathbf{F}$

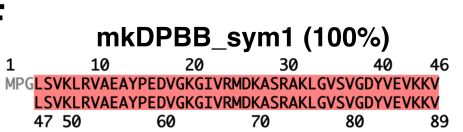

E

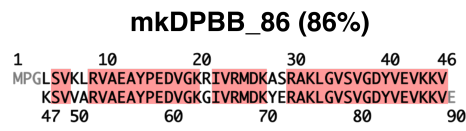

Cluster 2

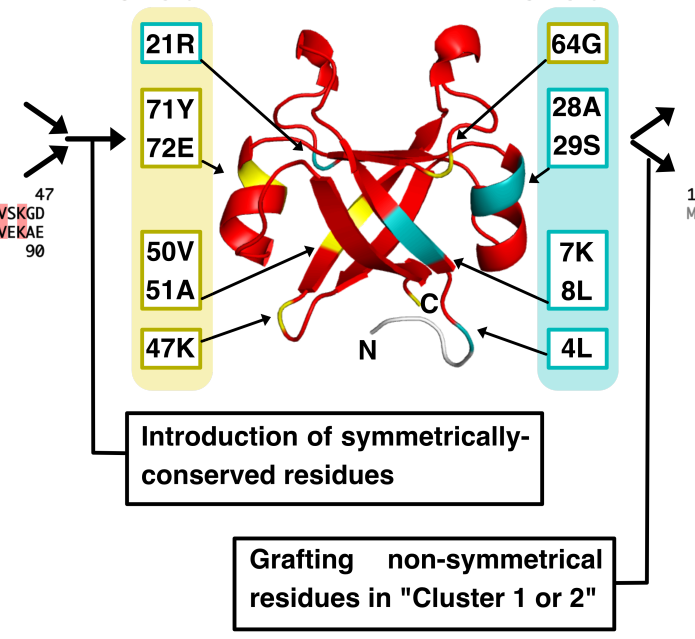

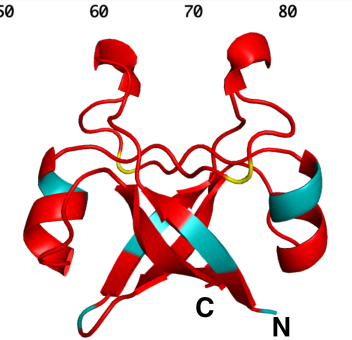

$$
\text { mkDPBB_sym2 (100\%) }
$$

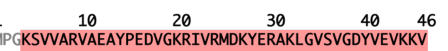
KSVVARVAEAYPEDVGKRIVRMDKYERAKLGVSVGDYVEVKKV

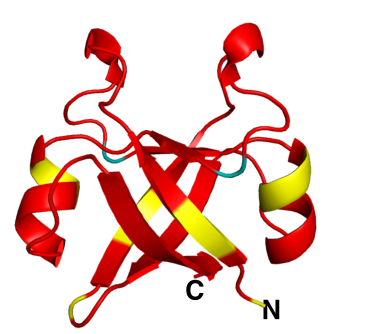

of panel A. The right view shows the left view rotated by 90 degrees around the horizontal axis.

(C-F) Symmetric-conservation design (SC-design). The amino acid sequence and crystal structure of (C) mkVCP_DPBB, (D) apVCP_DPBB, (E) mkDPBB_86, and (F) mkDPBB_sym1 and 2 are shown in each panel. The conserved residues in both halves are highlighted in red. (E) 
bioRxiv preprint doi: https://doi.org/10.1101/2021.02.22.432383; this version posted February 23, 2021. The copyright holder for this

preprint (which was not certified by peer review) is the author/funder. All rights reserved. No reuse allowed without permission.

mkDPBB_86 has only a limited number of non-symmetric positions, which are grouped in two areas (clusters 1 and 2). 
A

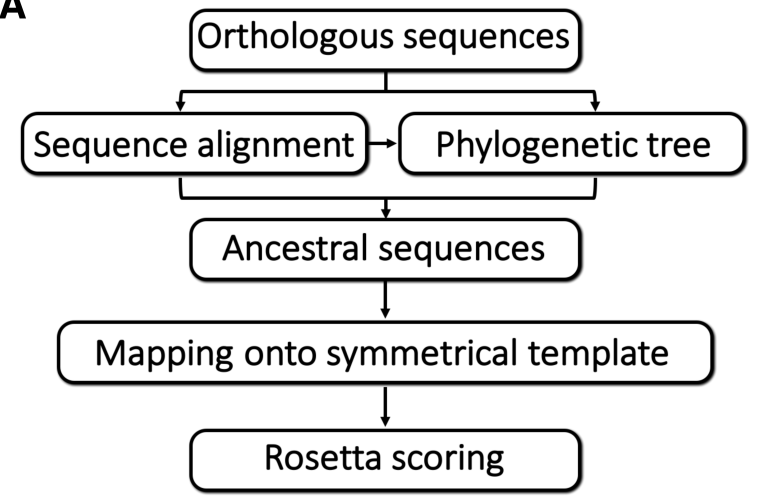

c

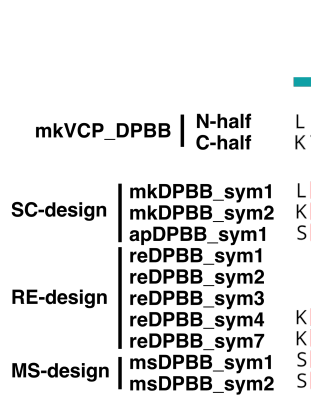

B

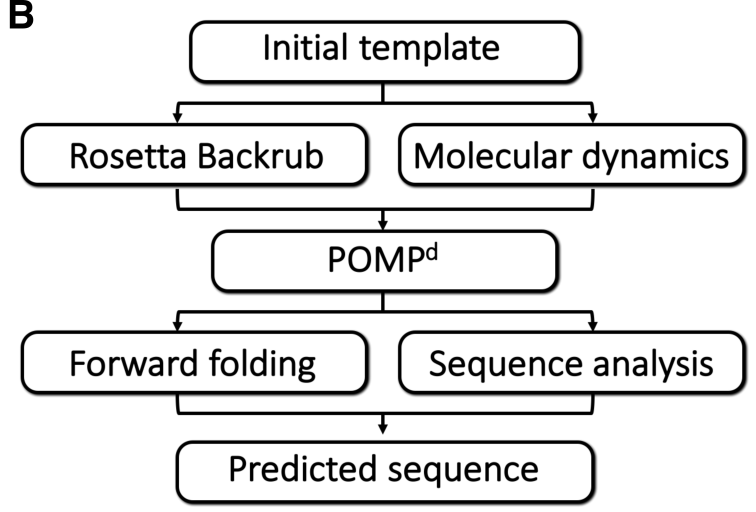

D

Figure 2. Computational design of symmetric DPBB and diversity of the symmetric DPBBs.

(A) Flow-chart of the RE-design scheme. (B) Flow-chart of the MS-design scheme. (C) Multiple sequence alignment of one repeat unit in the symmetric designs along with the mkVCP_DPBB, the starting template DPBB. The columns corresponding to the consensus residues in mkVCP_DPBB are highlighted in gray. The perfectly identical columns in the symmetric designs are colored red, and the columns with sequence identities over $60 \%$ are colored pink. (D) The perfectly conserved residues among all experimentally confirmed symmetric designs are mapped on the crystal structure of reDPBB_sym1. 
A

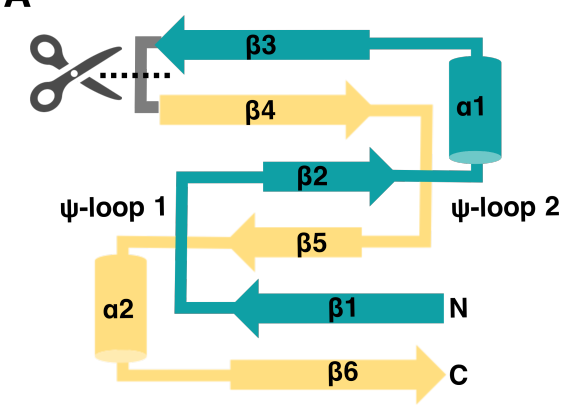

B

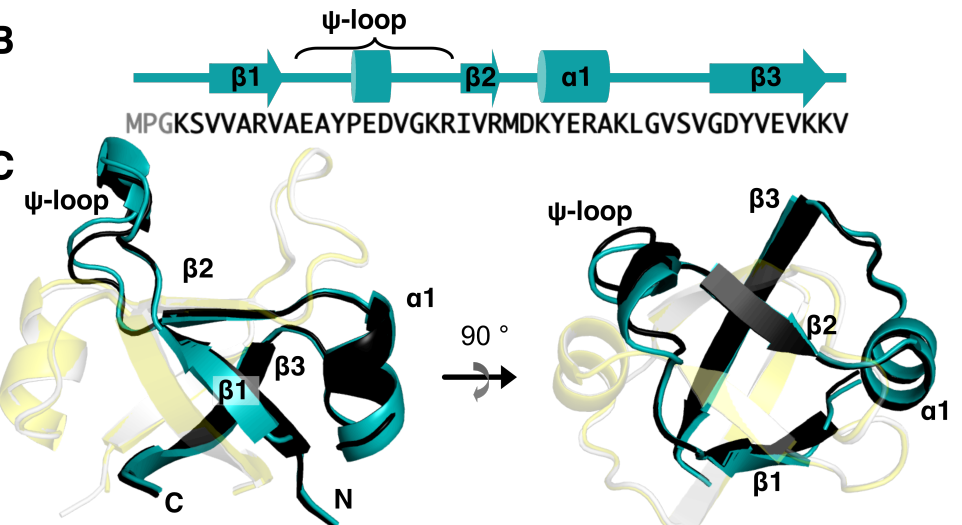

D

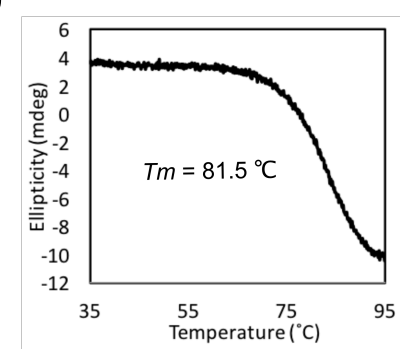

E
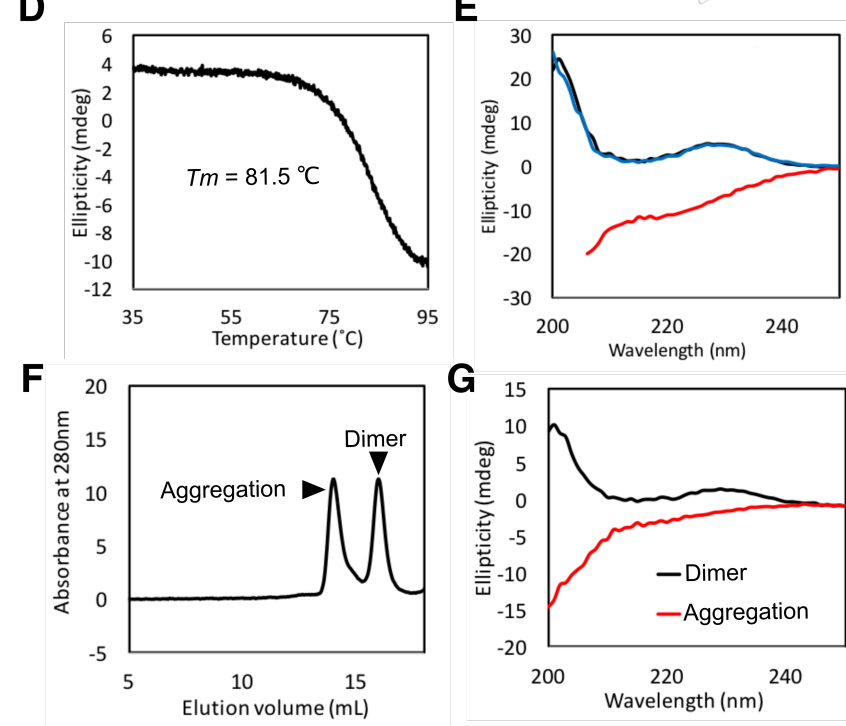

H

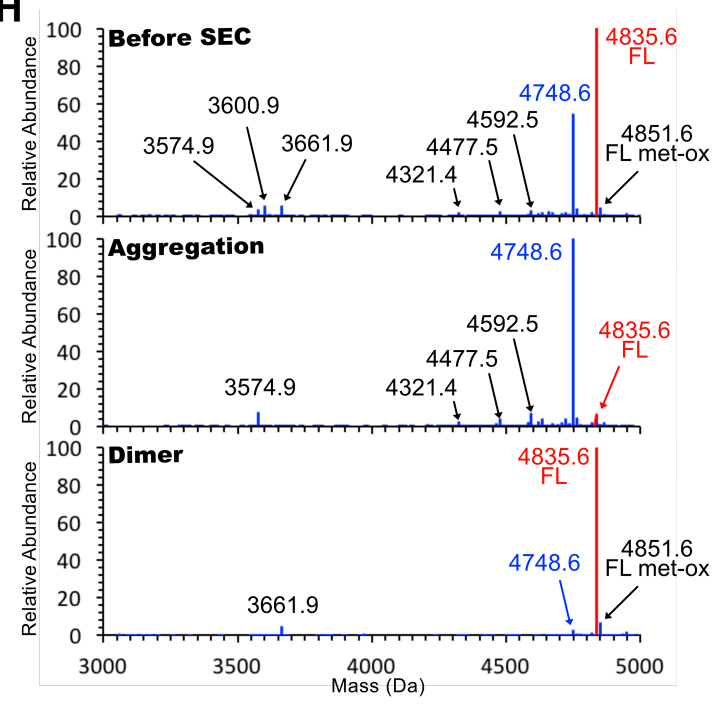

Figure 3. The DPBB fold formed by halved fragments. (A) Topology diagram of the halved fragments. (B) The sequence and secondary structure of mk2h. (C) The crystal structures of homodimeric $\mathrm{mk} 2 \mathrm{~h}$. The E. coli-produced peptides are colored cyan and yellow. The chemically synthesized peptides are colored black and white. (D and E) Thermostability and refolding ability of mk2h. (D) Denaturation was monitored by measuring the CD ellipticity at $222 \mathrm{~nm}$. (E) CD spectra at $35^{\circ} \mathrm{C}$ (black line), after heating to $95^{\circ} \mathrm{C}$ (red line), and upon cooling back to $35^{\circ} \mathrm{C}$ (blue line). (F-H) Folding of the low-purity mk2h sample (71.16\%). (F) SEC analysis showing that the dissolved $\mathrm{mk} 2 \mathrm{~h}$ peptide adopted aggregated and dimeric states. (G) CD spectra indicating that the aggregated and dimeric species, separated in Fig. 3F, adopt random-coil and $\alpha / \beta$ structures, respectively. $(\mathrm{H})$ The peptide species in the sample before and after SEC purification were 
analyzed by LC/MS. The deconvoluted mass spectra are shown. The labels for the full-length mk2h peptide (4835.6 Da) and the major contaminant peptide (4748.6 Da) are highlighted in red and blue, respectively. 


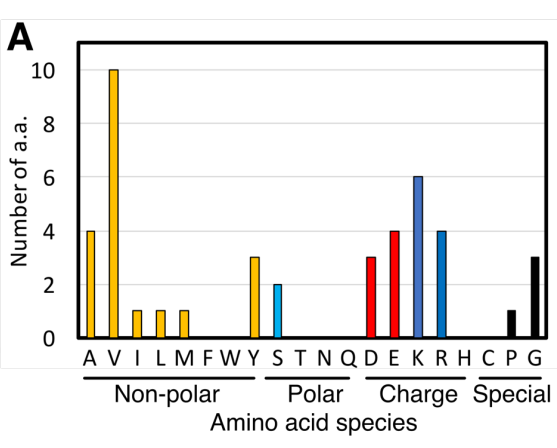

B

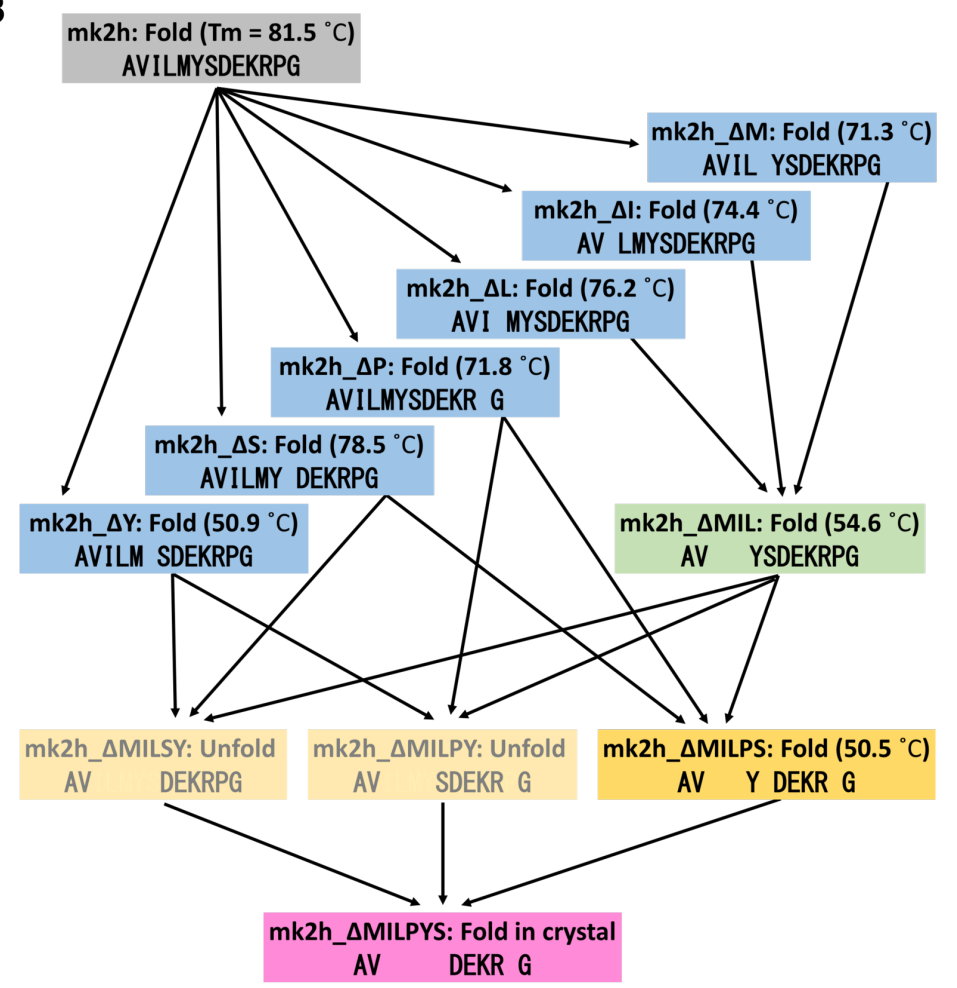

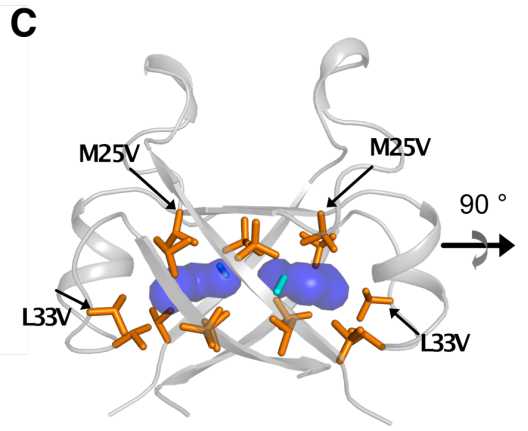

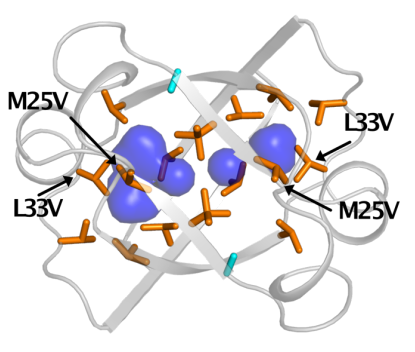

D

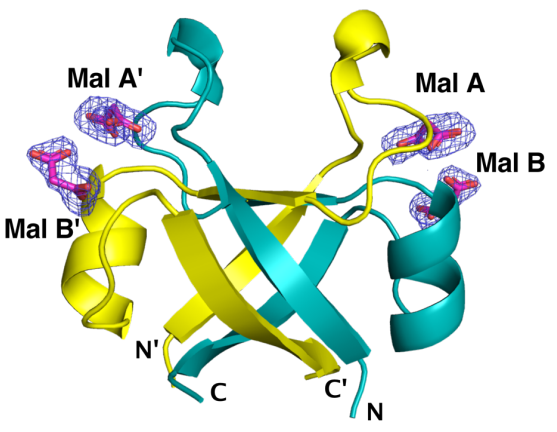

E

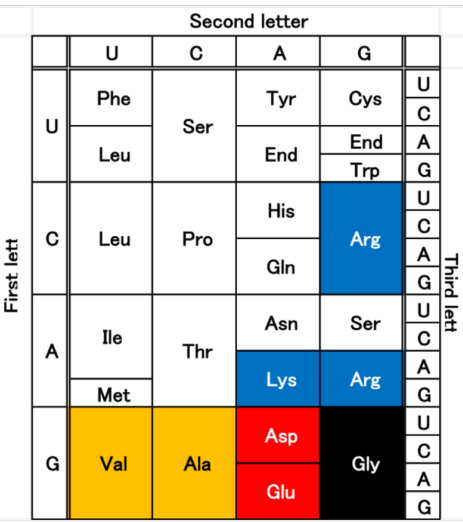

Figure 4. Reduction of amino acid repertory in $\mathbf{m k 2 h}$. (A) The amino acid usage in mk2h. (B)

Scheme of the design process of the simplified mk2h variants. (C) The hydrophobic core of mk2h_AMIL, composed of only Val (orange) and Ala (cyan). The substituted residues (M25V and L33V) from $\mathrm{mk} 2 \mathrm{~h}$ are indicated. The cavities in the core are shown as blue surface models. (D) The crystal structure of mk2h_AMILPYS. Four malonate ions, Mal A (A') and B (B') are bound to two symmetrical positions. The malonates are shown by stick models, and their Fo-Fc electron density omit maps are presented as a blue mesh (contoured at 1.0б). (E) The seven amino acid species used in mk2h_SMILPYS are highlighted on the standard codon table. 
Table 1. Summary of experimental tests for three different design strategies.

\begin{tabular}{ccccccc} 
& $\begin{array}{c}\text { Tested } \\
\text { designs }\end{array}$ & Express $^{\text {a }}$ & Soluble $^{\text {a }}$ & $\begin{array}{c}\alpha / \beta \\
\text { structure }\end{array}$ & Monomeric $^{\text {b }}$ & $\begin{array}{c}\text { Crystal } \\
\text { structure }\end{array}$ \\
\hline SC-design & 4 & 4 & 3 & 3 & 3 & 2 \\
RE-design & 7 & 5 & 5 & 5 & 5 & 3 \\
MS-design & 2 & 2 & 2 & 2 & 2 & 1 \\
\hline
\end{tabular}

${ }^{a}$ Expressions and solubilities were examined by SDS-PAGE.

${ }^{\mathrm{b}}$ Oligomeric states were examined by size exclusion chromatography. 


\section{SUPPLEMENTARY MATERIALS}

Materials and Methods

Supplementary Text

Figs. S1 to S20

5

Algorithm S1

Tables $\mathrm{S} 1$ to $\mathrm{S} 12$

References (1-44) 


\section{Materials and Methods}

\section{Identification of modern DPBB sequences with high internal symmetry}

Sequences of the molecular chaperone VCP were identified from the in-house protein database with reduced taxonomic bias (1) by searching with the BlastP program (2) using the taVCP_DPBB sequence as a query, and only the DPBB domains were extracted from the full-length VCP proteins. The 249 sequences thus obtained were aligned with the Muscle program. We fragmented the aligned sequences at the loop between the $\mathrm{N}$-half and $\mathrm{C}$-half $\beta \beta \alpha \beta$ elements, by referring to the structural information of taVCP_DPBB, and re-aligned them together. Based on the obtained multiple alignments, the internal sequence identities in each organism's DPBB were evaluated and ranked, as shown in Table S3.

\section{$\underline{\text { SC-design }}$}

Perfectly symmetric DPBBs were constructed by introducing mutations in a stepwise manner, using mkVCP_DPBB and apVCP_DPBB as templates. Initially, mkDPBB_sym_65 (internal sequence identity: 65\%) and apDPBB_sym_63 (63\%) were constructed by introducing the symmetrically-conserved residues in the N- and C-halves of mkVCP_DPBB and apVCP_DPBB into each other. The conserved residues in the other eight DPBBs with high internal sequence identities were then introduced into mkDPBB_sym_65 and apDPBB_sym_63 to construct mkDPBB_sym_79 (79\%) and apDPBB_sym_79 (79\%). The engineering step was repeated one more time using the sequence information from ten additional organisms, to construct mkDPBB_sym_86 (86\%) and apDPBB_sym_84 (84\%). mkDPBB_sym_86 and apDPBB_sym_84 have only a limited number of non-symmetrized positions, which are clustered in two areas in their tertiary structures. By adopting the amino acid residues from each of the two 
non-symmetrized areas, we then designed four DPBB proteins with perfect internal sequence symmetries (mkDPBB_sym1, mkDPBB_sym2, apDPBB_sym1, and apDPBB_sym2).

\section{$\underline{\text { RE-design }}$}

The design of completely symmetrical DPBB sequences was carried out using the "reverse engineering evolution" computational protein design approach (3). This method starts from the pseudo-symmetric sequences of each subunit, then constructs a phylogenetic tree, and subsequently generates putative ancestral sequences for further evaluation. This has been successfully applied to the design of symmetric proteins with 3-8 subunits $(4,5)$. However, DPBB is an extreme case with only 2 subunits, which makes the prediction of ancestral sequences based on the phylogenetic tree constructed from only 2 sequences challenging. To overcome this challenge, we have introduced the use of orthologous sequences instead of pseudo-symmetric sequences for the construction of phylogenetic trees. We first collected $498 \mathrm{VCP}$ sequences from different organisms and then removed the redundant sequences having $>75 \%$ sequence identity. The resulting sequences were aligned to generate a phylogenetic tree using a maximum-likelihood method (Jones-Taylor-Thornton (JTT) model) with 50-100 bootstrapping $(6,7)$. The aligned sequences and constructed phylogenetic tree were used together to generate putative ancestral consensus sequences by the FastML server, using a joint reconstruction substitution model (8). Approximately 28,000 ancestral sequences were then mapped onto a manually constructed, symmetrical mkVCP backbone structural model, and their energies were calculated using an inhouse "sequence_mapping.py" program that utilizes the PyRosetta of Rosetta protein modeling suite (9-11). This program uses an input list of plausible sequences and maps them onto a template protein backbone structure to output a PDB model for each sequence and the associated score. The top-scored designs were then analyzed for Rosetta total score, and root mean square deviations 
(RMSDs) from the symmetrical mkVCP backbone model structure. Finally, shortlisted designs were selected for experimental validation based on the Rosetta scores, RMSD from the design template, predicted solubility and visual inspection.

\section{$\underline{\text { MS-design }}$}

\section{1) $\operatorname{POMP}^{\mathrm{d}}$ (POsitive Multistate Protein design)}

POMP $^{\mathrm{d}}$ was used to compute minimum energy protein sequences from an ensemble of conformational states (12). Let us assume a rigid backbone and a pairwise decomposable energy function taking the form:

$$
E(s)=\sum_{i=1}^{n} E_{i}\left(s_{i}\right)+\sum_{i<j} E_{i j}\left(s_{i}, s_{j}\right)
$$

with $E(s)$ the total energy of protein sequence $s$ of length $n, E_{i}\left(s_{i}\right)$ a unary energy term for residue $s_{i}$ and $E_{i j}\left(s_{i}, s_{j}\right)$ a binary term representing energy interactions between residue pairs $\left(s_{i}, s_{j}\right)$. In this context, POMPd looks for the sequence that minimizes the energy of an ensemble of conformational states described as the sum of the energies on each state. POMPd models this problem as a cost function network and solves it exactly, using the constraint programming prover toulbar2, by returning the global minimum of the energy function and proving its optimality. This deterministic approach provides optimality guarantees: given an ensemble of conformational states and an energy function, the sequence returned is the global minimum of the energy function.

2) Conformational state ensemble preparation

mkVCP_sym2 crystal structure was used as the initial template. Two strategies were used in order to generate conformational states. In the first one, 100 protein models were generated using Rosetta Backrub protocol, with harmonic restraints on initial atoms coordinates. In the second one, 
a 100-ns molecular dynamics (MD) simulation at $300 \mathrm{~K}$ was performed starting from the initial template. Conformational states were extracted every $0.5 \mathrm{~ns}$, generating 200 protein models. For each strategy, the resulting protein models were clustered using Durandal (13). The clustering radius was set to $0.15 \AA$ for the first strategy and $0.5 \AA$ for the second. In each case, the cluster centers of the 4 biggest clusters were selected as the ensemble of conformational states. From these two strategies, we obtained two ensembles of 4 conformational states: the backrub ensemble and the MD ensemble.

3) Protein sequence predictions

POMP $^{d}$ was used to compute optimal protein sequences from backrub and MD ensembles using two different setups, which allowed the prediction of a total of four protein sequences. For both setups, we used the ability of the toulbar 2 prover to accept hard constraints in energy minimization to constrain sequences to be identical in each DPBB symmetrical subunit. In the first setup, all amino acid types were allowed at each position in a DPBB subunit. In the second setup, additional hard constraints were used in order to prevent the formation of solvent exposed hydrophobic patches.

While environmental factors such as $\mathrm{pH}$, ionic strength, temperature, and the presence of various solvent additives may influence protein solubility, internal factors are defined by the amino acids present at the protein surface (14). Protein solubility is determined by the amount of exposed hydrophobic surface area in the protein folded state $(15,16)$. Furthermore, the rate of aggregation of proteins and peptides increases as the amount of exposed hydrophobic surface area increases (17). Therefore, computational protein design tools must take surface hydrophobicity into account when designing new sequences. In order to do so, we limit the formation of exposed hydrophobic surface area by adding constraints in the form of new energy function terms. This functionality has 
been implemented in POMPd $^{\mathrm{d}}$ an additional feature called hpatch. The hpatch procedure is described in Supplementary Algorithm S1.

Forward folding experiments were performed on the four predicted sequences, using the protein structure prediction software EdaRose (18). Forward folding aims at assessing the quality of a protein design by predicting whether it will fold into the target structure or not. For each sequence, 30,000 structural models were predicted with EdaRose (Fig. S10). The number of iterations of EdaRose was set to 6, and the beta_nov16 scoring function from the Rosetta modeling software was used.

After examination of sequences and forward folding results, two sequences were selected for experimental characterization. The first one (msDPBB_sym1), from the backrub ensemble and using hpatch, was selected based on its forward folding profile. The second one (msDPBB_sym2), from the backrub ensemble and without using hpatch, was selected due to its sequence dissimilarity with other designs.

\section{$\underline{\text { Molecular dynamics simulations }}$}

All simulations were performed using the Amber ff14SB force field (19) implemented in the AMBER 16 package (20). To obtain a neutral charge of the simulated systems, several counterions were included. Each protein with the counter-ions was solvated with TIP3P water molecules, using an octahedral box with a minimum distance of $12 \AA$ between the solute and the simulation box edges. All systems were first subjected to 7 iterations of 1,000 minimization steps consisting of 500 steps of steepest descent minimization followed by 500 steps of conjugated gradient. A decreasing harmonic restraining potential was applied to the solute heavy atoms during the 6 first minimization iterations using a force constants of $100,50,20,10,5$, and 1 kcal.mol ${ }^{-1} . \AA^{-2}$ respectively. Heating of each system (NVT simulation) up to $300 \mathrm{~K}$ was carried out during $100 \mathrm{ps}$ 
under periodic boundary conditions, with positional restraints applied to the solute heavy atoms using a force constant of $25 \mathrm{kcal} \cdot \mathrm{mol}^{-1} . \AA^{-2}$. NVT simulation at the target temperature $(300 \mathrm{~K})$ was further conducted during $300 \mathrm{ps}$ in the same conditions. A $200 \mathrm{ps}$ simulation at constant pressure and temperature (NPT) was later performed to equilibrate the pressure of each system around the target value of 1 bar. During this step, a weak positional restraint was applied on the solute heavy atoms, using a force constant of $5 \mathrm{kcal} \cdot \mathrm{mol}^{-1} . \AA^{-2}$. An unrestrained MD simulation of $100 \mathrm{~ns}$ was finally performed under the same conditions. The Berendsen algorithm (21) was used to keep both temperature and pressure constant during simulations. A cutoff of $9 \AA$ was used to define longrange electrostatic interactions, which were calculated using the Particle Mesh Ewald algorithm (22). Bonds involving hydrogen atoms were constrained using the SHAKE algorithm (23) to enable the use of a 2 fs time step. All trajectory analyses were carried out with the CPPTRAJ module (24).

\section{Construction of expression vectors}

The synthetic genes encoding the proteins used in this study were purchased (Thermo Fisher Scientific, MA, USA). After amplifying the genes by PCR, each product DNA fragment was cloned using In-Fusion ${ }^{\mathrm{TM}}$ (TAKARA Bio, Japan) into the pET47b vector, to add a cleavable Nterminal His 6 -tag to the sequences. When sub-cloning the genes for a halved fragment, "XXX_half" and "Cloning_upstream" primers were used in the PCR amplification. The DNA sequences used in this study are listed in Supplementary Table S11.

\section{Protein expression and purification}

To produce the proteins, competent E. coli BL21-Gold(DE3) cells (Agilent Technologies, CA) were transformed with the respective expression vectors. The transformants were cultured at $37^{\circ} \mathrm{C}$ 
overnight in $20 \mathrm{~mL}$ of Luria Broth medium supplemented with $20 \mu \mathrm{g} / \mathrm{mL}$ kanamycin. The cells were then inoculated into $2 \mathrm{~L}$ of Luria Broth medium and cultured at $37^{\circ} \mathrm{C}$ for 2 hours. For induction, $0.5 \mathrm{mM}$ isopropyl $\beta$-D-1-thiogalactopyranoside (IPTG) was added to the media, and the desired proteins were expressed for 4 hours under the same conditions. After harvesting the cells, the pellets were resuspended in $60 \mathrm{~mL}$ of $50 \mathrm{mM}$ potassium phosphate buffer, $\mathrm{pH} 6.5,150$ $\mathrm{mM} \mathrm{NaCl}$, and sonicated. The bacterial lysate was fractionated into supernatant and precipitant by centrifugation at $8,000 \mathrm{rpm}$ and $4{ }^{\circ} \mathrm{C}$, for $20 \mathrm{~min}$. To precipitate the contaminating E. coli proteins, the supernatants were incubated at $70{ }^{\circ} \mathrm{C}$ for $20 \mathrm{~min}$, and then each soluble fraction was isolated by centrifugation at $8,000 \mathrm{rpm}$ and $4{ }^{\circ} \mathrm{C}$ for $20 \mathrm{~min}$. In the cases of mk2h_AMILPS,

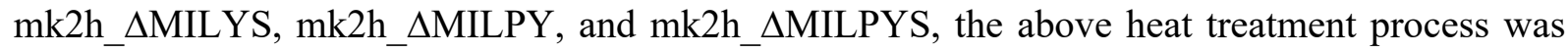
omitted in order to preserve their native structures. The soluble proteins were purified by HisTrap HP nickel affinity chromatography (GE Healthcare, IL). The N-terminal His 6 -tags were cleaved with HRV-3c protease (Funakoshi, Japan) at $4^{\circ} \mathrm{C}$ for $1-2$ days. To remove the cleaved His6-tag and residual uncleaved proteins, the treated solutions were loaded onto HisTrap columns, and each flow-through fraction was recovered. The protein solutions were additionally loaded onto a HiLoad 16/600 Superdex75 (GE Healthcare, IL) size exclusion chromatography column, equilibrated with $50 \mathrm{mM}$ potassium phosphate buffer, $\mathrm{pH} 6.0,150 \mathrm{mM} \mathrm{NaCl}$, and the peak fractions were collected. The purity of each protein was verified by SDS-PAGE, and the protein concentrations were determined using their $\mathrm{A}_{280}$ values, measured with an ultraviolet spectrophotometer (NanoDrop, Thermo Scientific).

For the preparation of seleno-methionine (Se-Met) substituted taVCP_DPBB, E. coli BL21Gold(DE3) cells were grown in $2 \mathrm{~L}$ of M9 minimal medium containing $20 \mu \mathrm{g} / \mathrm{L}$ of kanamycin at $37{ }^{\circ} \mathrm{C}$ until they reached an absorbance at $600 \mathrm{~nm}\left(A_{600}\right)$ of 0.4 . An amino acid mixture $(50 \mathrm{mg} / \mathrm{L}$ isoleucine, leucine and valine and $100 \mathrm{mg} / \mathrm{l}$ phenylalanine, threonine and lysine) and seleno-L- 
methionine $(60 \mathrm{mg} / \mathrm{mL})$ were then added to the culture, and the cells were grown at $37^{\circ} \mathrm{C}$. After reaching an $A_{600}$ of 0.8 , the protein expression was induced with $0.5 \mathrm{mM}$ IPTG, and the cells were grown further at $37^{\circ} \mathrm{C}$ for 5 hours.

\section{Biophysical characterization}

For the gel filtration analysis, the concentrations of full-length proteins and halved fragments were adjusted to $20 \mu \mathrm{M}$ and $40 \mu \mathrm{M}$, respectively. A $100 \mu \mathrm{l}$ aliquot of each purified protein was applied to a Superdex 75 Increase 10/300 size exclusion chromatography column, equilibrated with $50 \mathrm{mM}$ potassium phosphate buffer, $\mathrm{pH} 6.0,150 \mathrm{mM} \mathrm{NaCl}$, and run on an AKTA FPLC (Amersham Biosciences) at a flow rate of $0.75 \mathrm{~mL} / \mathrm{min}$.

CD spectra were collected on a JASCO J820 circular dichroism spectrometer (JASCO, Japan). Samples containing $20 \mu \mathrm{M}$ full-length proteins or $40 \mu \mathrm{M}$ halved fragments, in $50 \mathrm{mM}$ potassium phosphate, $\mathrm{pH} 6.0,150 \mathrm{mM} \mathrm{NaCl}$, were loaded into a $1 \mathrm{~mm}$ pathlength quartz cuvette. Spectra were recorded in the wavelength range from 200 to $250 \mathrm{~nm}$ at $1 \mathrm{~nm}$ intervals at $25^{\circ} \mathrm{C}$, and each spectrum was the average of 10 scans. Spectra for mk2h mutants containing 7, 8, or 10 amino acid repertoires were recorded at $10{ }^{\circ} \mathrm{C}$.

The melting curves were collected on a JASCO J820 CD spectrometer monitored at $222 \mathrm{~nm}$, in $50 \mathrm{mM}$ potassium phosphate buffer, $\mathrm{pH} 6.0,150 \mathrm{mM} \mathrm{NaCl}$. The temperature was increased at a rate of $1.0{ }^{\circ} \mathrm{C} / \mathrm{min}$. Data points were collected at $0.1{ }^{\circ} \mathrm{C}$ increments from $35{ }^{\circ} \mathrm{C}$ to $95{ }^{\circ} \mathrm{C}$, or from $10{ }^{\circ} \mathrm{C}$ to $95^{\circ} \mathrm{C}$.

\section{Crystallography}

For crystallization, all purified protein solutions were dialyzed against $20 \mathrm{mM}$ Bis-tris $\mathrm{HCl}, \mathrm{pH}$ $6.0,150 \mathrm{mM} \mathrm{NaCl}$ and then concentrated to $30-70 \mathrm{mg} / \mathrm{ml}$. Crystallization screenings were carried 
out in 96-well sitting-drop vapor-diffusion plates. Sample solutions $(200 \mathrm{~nL})$ were mixed with an equal amount of reservoir solutions and incubated at $20^{\circ} \mathrm{C}$. Almost all crystals were obtained after a few hours to a few days. The crystals were cryo-cooled using the reservoir solution including 13-30\% glycerol as a cryo-protectant (Table S12).

Data were collected at the Photon Factory (Tsukuba, Japan)(25, 26), SPring 8 (Harima, Japan)(27-32), or Swiss Light Source (Villigen, Swiss). The beam lines and detectors are listed in Table S9. The X-ray diffraction data were processed with XDS (33). All structures were solved and refined with the program PHENIX $(34,35)$. The structures of taVCP_DPBB, mkVCP_DPBB, and apVCP_DPBB were solved by the SAD phasing method, using phenix.autosol. The initial structure models for other mutants were determined by the MR phasing method, using phenix.phaser-MR and the crystal structures of DPBB determined in this study as the search models. The model structures were updated manually using Coot (36) and iteratively refined with Phenix.refine. Statistics for diffraction data collection and refinement are summarized in Table S2.

\section{Characterization of chemically-synthesized peptides}

All chemically-synthesized peptides tested in this report were obtained from Japan Bio Services. The synthesized mk2h (95.14\% purity) and mk2h_AMILPS $(95.56 \%)$ peptides in powdered forms were dissolved in $20 \mathrm{mM}$ Bis-tris $\mathrm{HCl}, \mathrm{pH} 6.0,150 \mathrm{mM} \mathrm{NaCl}$. The concentrations were determined by the absorbance at $280 \mathrm{~nm}$, using an ultraviolet spectrophotometer (NanoDrop One, Thermo Scientific). For the crystallization screenings, the mk2h_dMILPYS peptide (95.85\%) was dissolved in $20 \mathrm{mM}$ Tris- $\mathrm{HCl}, \mathrm{pH} 8.5,200 \mathrm{mM}$ lithium sulfate. The dissolved peptide sample was separated into supernatant and precipitate fractions, and then the precipitate was resuspended in the same buffer. Both the supernatant and undissolved suspension were used for the crystallization screenings, which were carried out in 96-well sitting-drop vapor-diffusion 
plates. The obtained crystals were processed in the same way as the bacteria-produced proteins described above.

To examine the foldability in the presence of the contaminated sequences, the low purity peptides of mk1h (75.06\%) and mk2h (71.16\%) were dissolved in $20 \mathrm{mM}$ Bis-tris $\mathrm{HCl}, \mathrm{pH} 6.0$, $150 \mathrm{mM} \mathrm{NaCl}$. The aggregated and dimer species were separated on a size exclusion chromatography column (Superdex 75 Increase 10/300) equilibrated with $20 \mathrm{mM}$ Bis-tris HCl, pH $6.0,150 \mathrm{mM} \mathrm{NaCl}$. The peptide concentrations of three samples, 1) before SEC purification, 2) aggregated fraction, and 3) dimer fraction, were adjusted to $20 \mu \mathrm{M}$ and $10 \mu \mathrm{M}$ for $\mathrm{CD}$ spectrum measurements and liquid chromatography mass spectrometry, respectively. Using a JASCO J820 circular dichroism spectrometer (JASCO, Japan), CD spectra were recorded at wavelengths from 200 to $250 \mathrm{~nm}$ at $1 \mathrm{~nm}$ intervals at $25^{\circ} \mathrm{C}$, and each spectrum was the average of 10 scans.

\section{$\underline{\text { LC-MS analysis }}$}

The peptide solutions were desalted with in-house made C18 stage-tips, dried under a vacuum, and dissolved in $2 \%$ acetonitrile and $0.1 \%$ formic acid. The 10 pmol peptide mixtures were fractionated by $\mathrm{C} 18$ reverse-phase chromatography $(1.8 \mu \mathrm{m}$, ID $0.075 \mathrm{~mm}$ x $250 \mathrm{~mm}$, Aurora UHPLC Column; IonOpticks, ADVANCE UHPLC; AMR Inc.) and applied directly into a hybrid linear ion trap mass spectrometer (LTQ Orbitrap Velos Pro; Thermo Fisher Scientific). The peptides were eluted at a flow rate of $200 \mathrm{~nL} / \mathrm{min}$ with a linear gradient of 5-35\% solvent B over 20 min. The compositions of Solvent A and B were 0.1\% TFA in water and 100\% acetonitrile, respectively.

The Orbitrap mass spectrometer was programmed to carry out 4 successive scans, with the first consisting of a full MS scan from $350-2,000 \mathrm{~m} / \mathrm{z}$ at a resolution of 60,000 , and the second to fourth consisting of data - dependent scans of the top three most abundant ions obtained in the first scan, 
at a resolution of 7,500. Automatic MS/MS spectra were obtained from the highest peak in each scan, by setting the relative collision energy to $35 \%$ and the exclusion time to $90 \mathrm{~s}$ for molecules in the same $\mathrm{m} / \mathrm{z}$ value range. Calculations of peptide masses and intensities in the time range from 15.0-35.0 min were performed with the Xtract deconvolution algorithm in FreeStyle, version 1.5 (Thermo Fisher Scientific). 


\section{Supplementary Text}

\section{$\underline{\text { SC-design }}$}

Most of the amino acid residues conserved between the N- and C-halves in each VCP_DPBB have probably remained unchanged from their perfectly symmetric ancestor, as the chance of having the same amino acid residues in the symmetric positions by random mutation is low. This idea is supported by the observation that the symmetrically-conserved residues in the DPBB domain from each archaeon are often shared with other species (Fig. S4). Studies have also shown that M. kandleri, the organism possessing one of the highest symmetric extant DPBBs, is close to the phylogenetic root of archaea (37-39).

We used mkVCP_DPBB and apVCP_DPBB, with the highest symmetrical sequence identities among the DPBB domains in our database, as the starting templates to create perfectly symmetrical DPBBs. Initially, the symmetrically-conserved residues in mkVCP_DPBB or apVCP_DPBB were introduced into each other to construct the chimeric DPBBs, mkDPBB_sym_67 (internal sequence identity: 67\%) and apDPBB_sym_63 (63\%) (Table S1). We confirmed that both proteins were monomers with an $\alpha / \beta$ structure (Fig. S5A and C). We then introduced the symmetricallyconserved residues in the other eight VCP_DPBB sequences top-ranked by internal sequence identities (Fig. S4 and Table S3) into mkDPBB_sym_67 and apDPBB_sym_63, resulting in mkDPBB_sym_81 (81\%) and apDPBB_sym_79 (79\%). Additionally, the symmetricallyconserved residues in ten more VCP_DPBB sequences were introduced, to construct mkDPBB_sym_86 (86\%) and apDPBB_sym_84 (84\%). Three proteins, except for mkDPBB_sym_81, were verified to be folded (Fig. S5B, D, and E). X-ray-crystallography confirmed that mkDPBB_sym_86 and apDPBB_sym_79 adopt the DPBB fold (Figs. 1E, S6A). The mkDPBB_sym_86 and apDPBB_sym_84 proteins have only a limited number of non- 
symmetrized positions, which are clustered in two areas. In mkDPBB_sym_86, cluster-1 comprises $4 \mathrm{~L}, 7 \mathrm{~K}, 8 \mathrm{~L}, 28 \mathrm{~A}, 29 \mathrm{~S}$, and $64 \mathrm{G}$ around $\alpha$-helix 1 and $\beta$-strand 1', and cluster-2 comprises 21R, 47K, 50V, 51A, 71Y, and 72E around $\alpha$-helix 1' and $\beta$-strand 1 (Fig. 1E). Looking at the overall structure of the original full-length VCP containing the other domains, these symmetrical faces in the DPBB domain are in different environments: the residues at cluster-1 are exposed to the solvent, while the residues at cluster-2 contact another domain (Fig. S7). This difference in the molecular environments probably led to a breakdown of the symmetry in this area during the evolutionary process.

Subsequently, we designed four DPBB domains with perfect internal sequence symmetries (mkDPBB_sym1, mkDPBB_sym2, apDPBB_sym1, and apDPBB_sym2) by adopting the amino acid residues from either cluster- 1 or -2 . While apDPBB_sym2 could not be purified due to its poor stability, mkDPBB_sym1, mkDPBB_sym2, and apDPBB_sym1 were purified as stable proteins (Fig. S5F-H). We determined the crystal structures of mkDPBB_sym1 and mkDPBB_sym2 to confirm that they adopt the DPBB fold as designed (Fig. 1F, Table S2). Therefore, we succeeded in reconstructing the perfectly symmetrical DPBB structures, using the sequence information of VCP-like chaperones from only twenty archaeal species. This result led us to anticipate that the archaeal common ancestor possessed a nearly perfect symmetric DPBB sequence in the VCP-like chaperone gene. Furthermore, all of the perfectly symmetrical DPBB and intermediate mutants constructed by the SC-design exhibited high stability. Except for mkDPBB_sym_67 $\left(T_{m}=69.2^{\circ} \mathrm{C}\right)$, they did not completely unfold even at $95^{\circ} \mathrm{C}$ (Fig. S5). These results support the hypothesis that the common ancestor of archaea is a thermophile (40).

\section{$\underline{\text { RE-design }}$}


We utilized a modified "reverse engineering evolution" protein design approach to design the symmetrical DPBB sequences. In this design methodology, orthologous sequences of the target protein are used instead of pseudo-symmetric sequences for the construction of phylogenetic trees. Specifically, we used VCP_DPBB sequences from different organisms to construct a phylogenetic tree with respective aligned sequences, which were subsequently used as input to generate ancestral sequences (Figure S8A and B). The predicted ancestral sequences were mapped onto a manually constructed, perfectly symmetrical mkVCP_DPBB structural backbone model using an in-house program that utilizes PyRosetta, and each sequence was ranked by the Rosetta score (Figure S8C and D). The top-scored designs were analyzed for Rosetta total score, RMSD from the manually generated, symmetrical mkVCP_DPBB model and through visual inspection. First, our analysis of the Rosetta total score revealed that many output models showed significantly lower energy and converged well (Figure S9A). When the backbone RMSDs of the designs were computed and plotted against the Rosetta total score, a broad spread of total score/RMSD scores was obtained. However, the majority of the top-scored output models exhibited RMSDs $<1 \AA$, suggesting even with diverse sequences, they did not deviate much from the starting structure (Figure S9B). Interestingly, several of the top-scored models exhibited RMSDs $<0.7 \AA$. The reDPBB_sym1 and reDPBB_sym2 exhibited $0.65 \AA$ and $0.64 \AA$ RMSD from the manually generated symmetrical mkVCP_DPBB model, respectively. Next, an analysis of Rosetta total score versus percentage sequence identity revealed that the top-scored structures tend to have higher percentage sequence identity (Figure S9C). However, the shortlisted reDPBB_sym1 and reDPBB_sym 2 designs shared a moderate $78 \%$ and $74 \%$ sequence identity with the manually generated, symmetrical mkVCP_DPBB model respectively.

We compared the Rosetta generated structural models to that of the crystal structures of reDPBB_sym1 and reDPBB_sym2. First, we found that in both reDPBB_sym1 and 
reDPBB_sym2 crystal structures, each half of the proteins exhibit $0.41 \AA$ RMSD with each other (Figures S9D-G). Moreover, while the crystal structure of reDPBB_sym1 and the Rosetta generated model of reDPBB_sym1 share 0.66 A RMSD, the reDPBB_sym2 crystal structure and Rosetta generated model of reDPBB_sym2 share $0.58 \AA$ RMSD (Figures S9H and S9I). This indicated that the Rosetta- generated structural models are in close agreement with the crystal structures. This result demonstrated that our computational symmetric design approach can be successfully applied to the design of DPBB fold as well.

Our designed reDPBB_sym1 and reDPBB_sym 2 sequences share $67 \%$ and $65 \%$ sequence identity with mkVCP_DPBB, and 44\% identity with taVCP_DPBB respectively. This further confirms that diverse sequences can fold into DPBB structures, as predicted from our computational symmetric protein design approach. Our computational strategy, of using orthologous sequences to construct phylogenetic trees for the design of symmetric proteins, has advantages over that of using pseudo-symmetric sequences from each subunit of the target protein. It circumvents the challenges when the number of subunits in the target protein is very small, such as the two subunits in DPBB. The successful computational design of symmetric DPBB proteins has verified the applicability of our design strategy under this circumstance. 
A

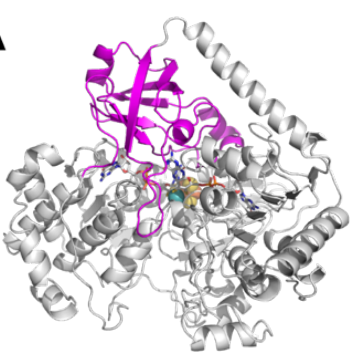

D

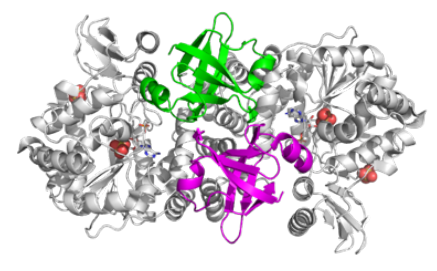

G

RNAP_DPR⿴囗十 taVCP_DPBB

$C_{a}$ RMSD= $1.6 \AA$

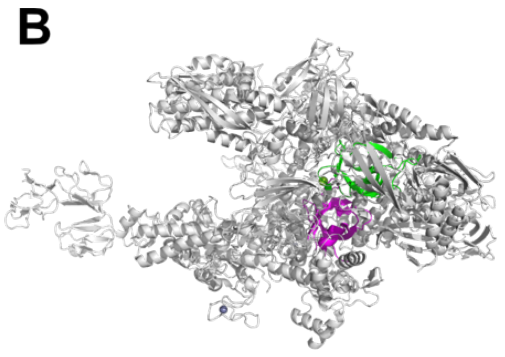

C

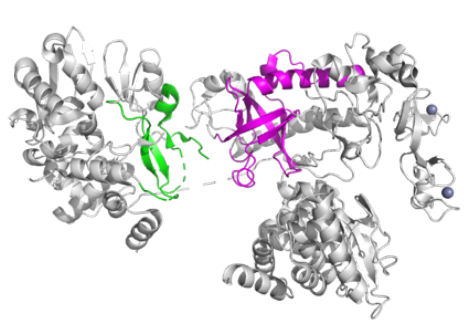

E

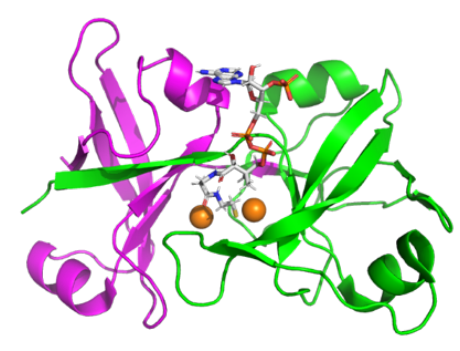

$\mathbf{F}$

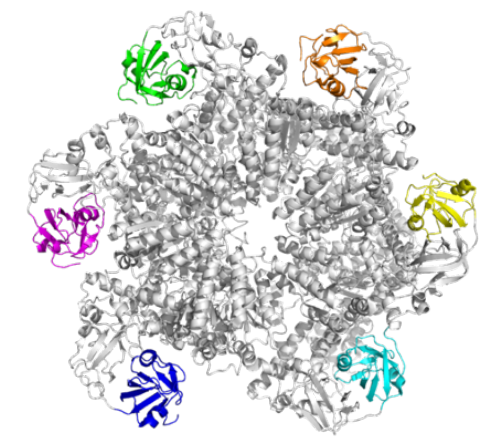

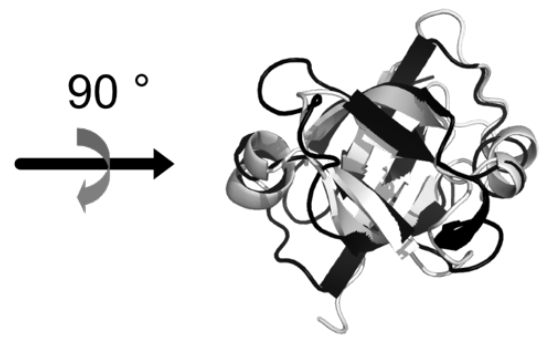

Figure S1. DPBB domains in natural proteins. Structures of $(\mathrm{A})$ formate dehydrogenase (PDB ID 1FDO), (B) RNA polymerase (PDB ID 6ASG), (C) D-type DNA polymerase (PDB ID 5IJL), (D) AMP phosphorylase (PDB ID 4GA6), (E) phosphate propanoyltransferase (PDB ID 5CUO), and (F) molecular chaperone VCP (PDB ID 5G4F) are shown. Each DPBB domain is colored differently. (G) The superimposed structures of the DPBB domain-2 from RNA polymerase (colored pink in Fig. S1B) and the isolated DPBB domain of molecular chaperone VCP (Fig. 1B). The C $\alpha$ RMSD was calculated by CRICK $(41,42)$ (http://cospi.iiserpune.ac.in/click/). 
A

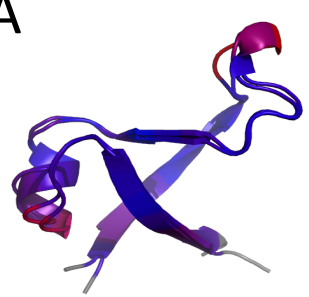

$0.94 \AA$
B

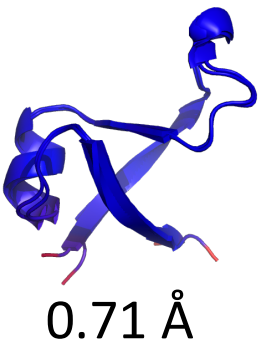

C

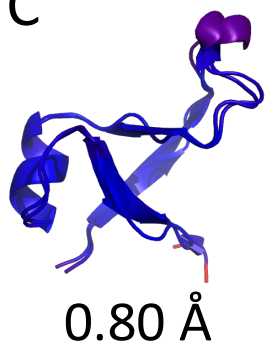

Figure S2. Superimposed structures of the N- and C-terminal halves of extant VCP_DPBBs.

Superimposition of the N- and C-terminal halves of the crystal structures of (A) taVCP_DPBB, (B) mkVCP_DPBB, and (C) apVCP_DPBB. Darker blue indicates better alignments in the structures. The values are the C $\alpha$ RMSDs calculated by CRICK $(41,42)$ (http://cospi.iiserpune.ac.in/click/). 
A
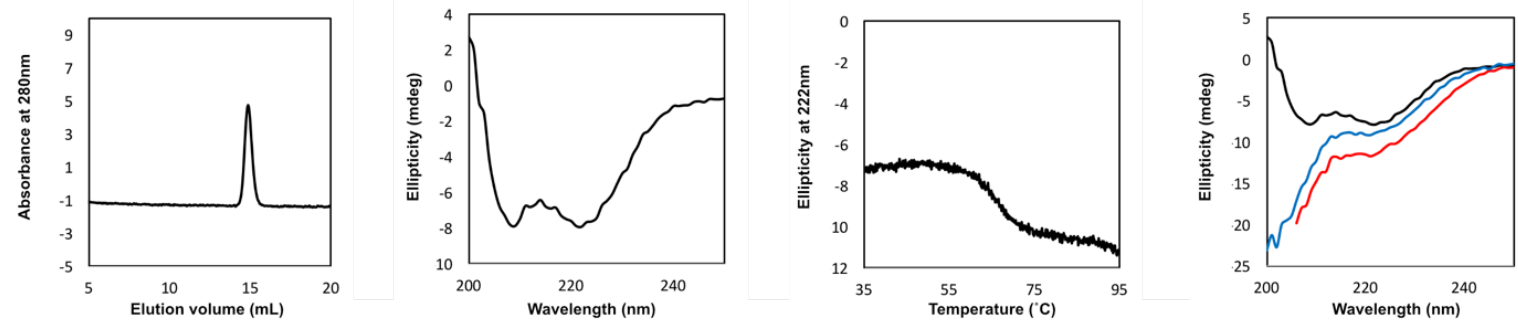

B
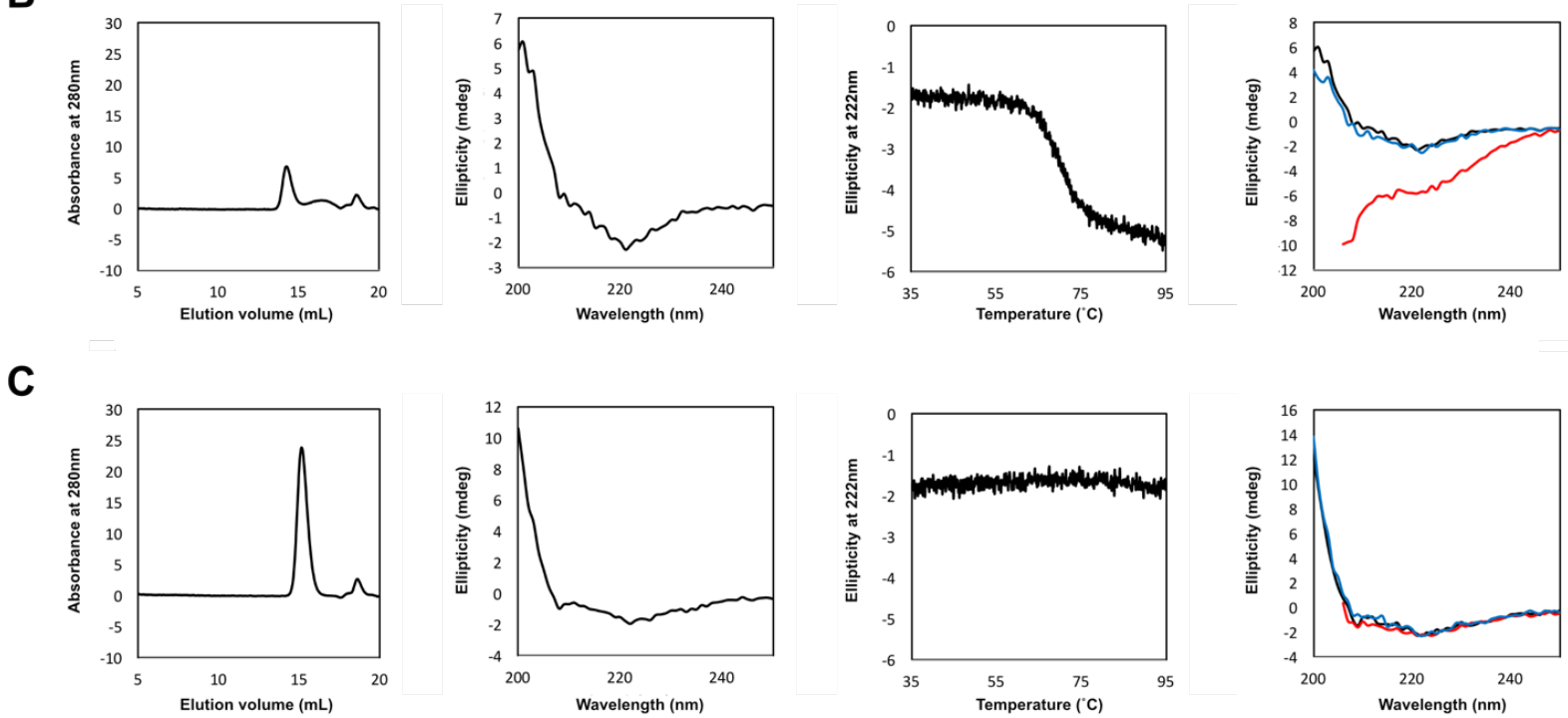

Figure S3. Experimental characterization of extant VCP_DPBBs. Size exclusion chromatography, CD spectra, denaturation curves, and comparisons of CD spectra at different temperatures (black: $35^{\circ} \mathrm{C}$; red: $95^{\circ} \mathrm{C}$; blue (refolding): $95^{\circ} \mathrm{C} \rightarrow 35^{\circ} \mathrm{C}$ ) for $(\mathrm{A})$ taVCP_DPBB, (B) mkVCP_DPBB, and (C) apVCP_DPBB are shown in the panels from left to right. 

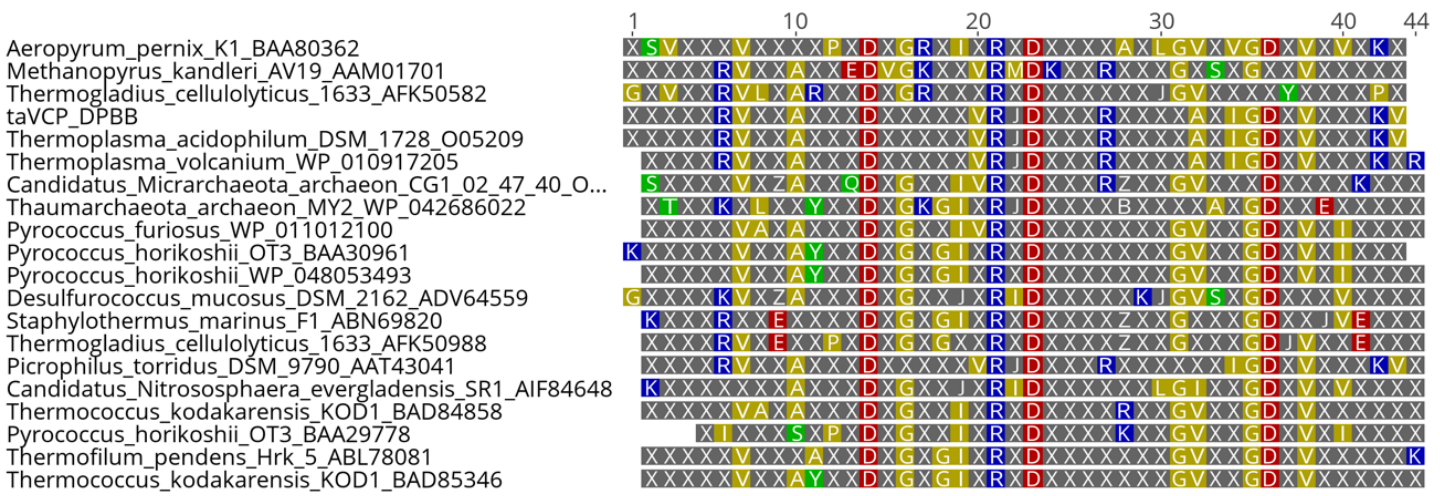

Figure S4. The symmetrically-conserved residues in natural VCP_DPBB domains. The symmetrically-conserved residues between the $\mathrm{N}$ - and $\mathrm{C}$-halves in each 20 top-ranked VCP_DPBB with high internal sequence identity are highlighted. The non-symmetricallyconserved residues are represented as $\mathrm{X}$ (gray). The sequences are ordered by the internal sequence identity shown in Table S9. To create mkDPBB_sym_81 and apDPBB_sym_79, the symmetrically-conserved residues in the top ten sequences were considered. To create mkDPBB_sym_86 and apDPBB_sym_84, the symmetrically-conserved residues of all sequences were considered. 
A
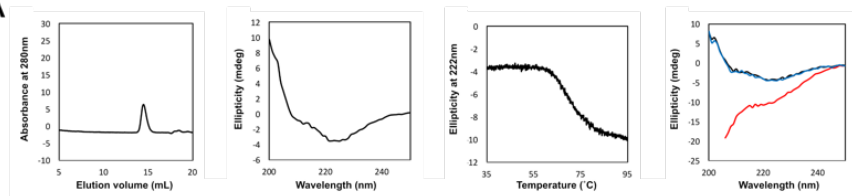

B
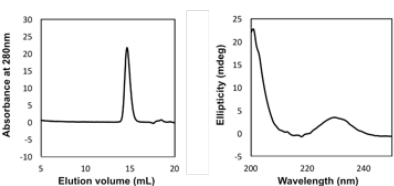

C
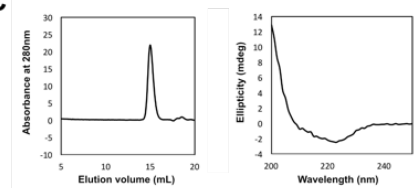

D
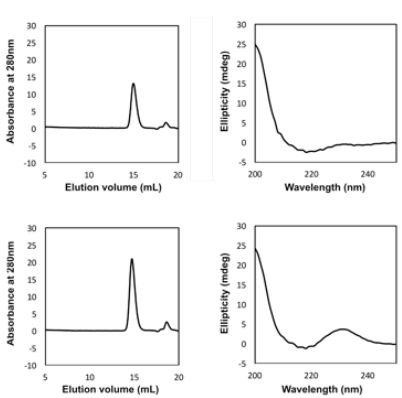

$\mathbf{F}$
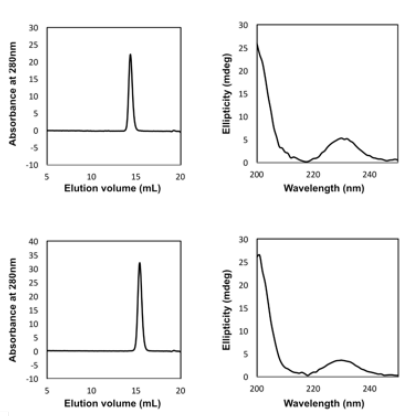

H

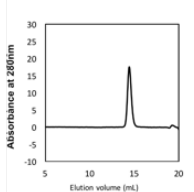

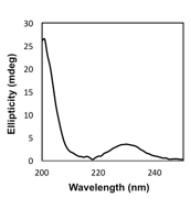

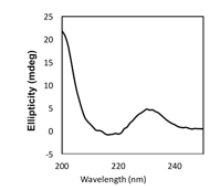

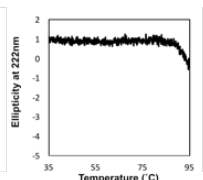
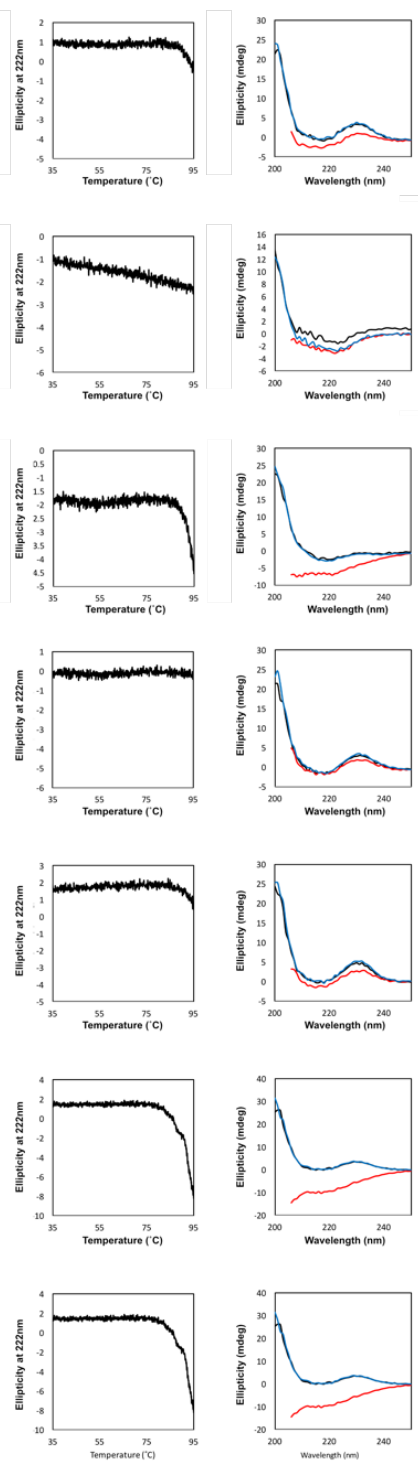

Figure S5. Experimental characterization of symmetrical DPBBs constructed by the SC-

design method. Size exclusion chromatography, CD spectra, denaturation curves, and comparisons of $\mathrm{CD}$ spectra at different temperatures (black: $35^{\circ} \mathrm{C}$; red: $95^{\circ} \mathrm{C}$; blue (refolding): $95^{\circ} \mathrm{C} \rightarrow 35^{\circ} \mathrm{C}$ ) for (A) mkDPBB_sym_67, (B) mkDPBB_sym_86, (C) apDPBB_sym_63, (D) 
apDPBB_sym79, (E) apDPBB_sym_84, (F) mkDPBB_sym1, (G) mkDPBB_sym2, and (H) apDPBB_sym1 are shown in the panels from left to right. 


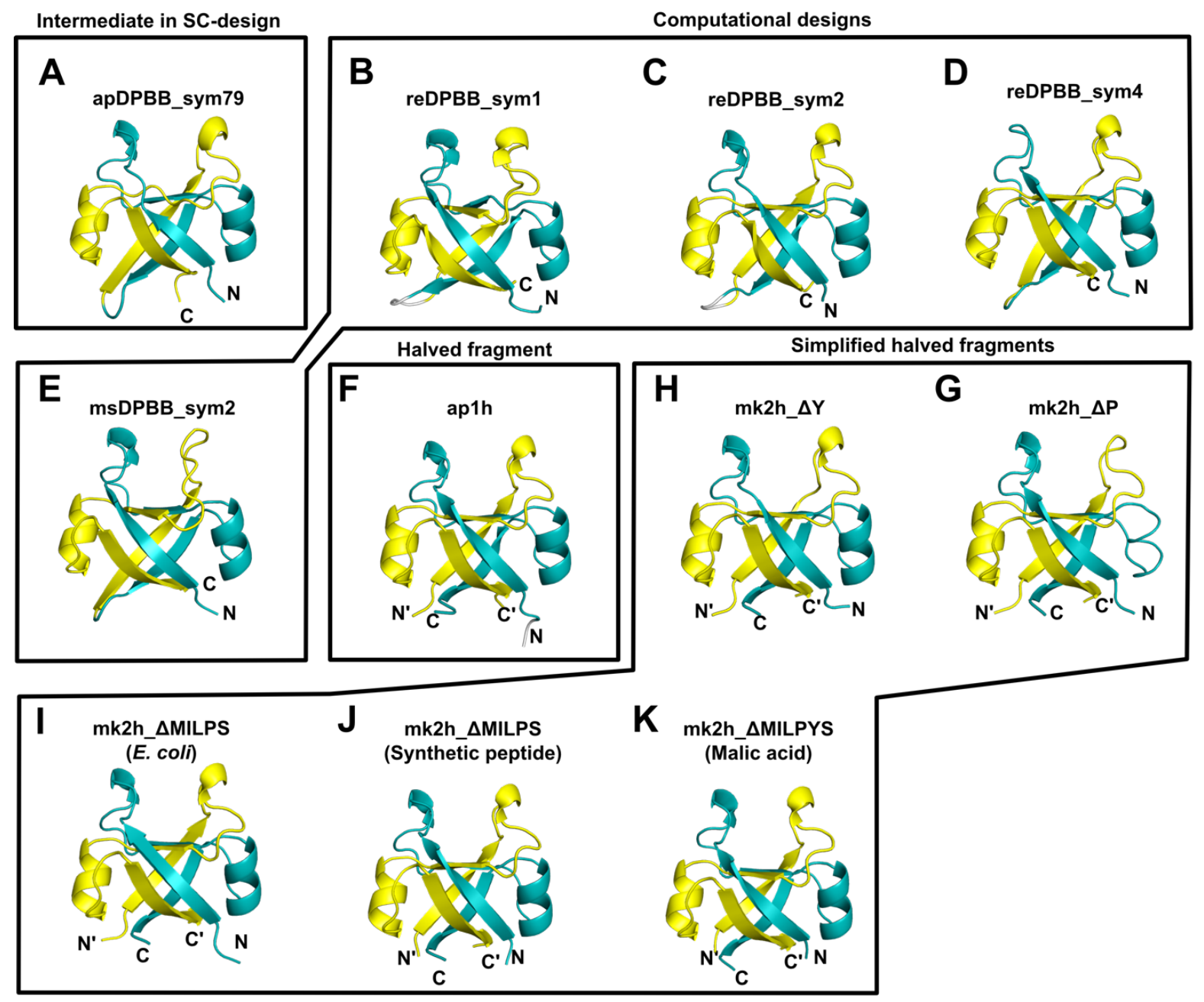

Figure S6. Crystal structures of the designed DPBB domains. (A) apDPBB_sym_79 (PDB ID 7DI0), (B) reDPBB_sym1 (7DVC), (C) reDPBB_sym2 (7DVF), (D), reDPBB_sym4 (7DVH), (E)

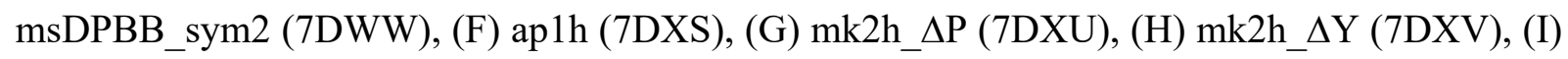
E. coli-produced mk2h_AMILPS (7DXX), (J) chemically-synthesized mk2h_AMILPS (7DXY), and (K) chemically-synthesized mk2h__MILPYS in the presence of DL-malic acid (7DYC). 


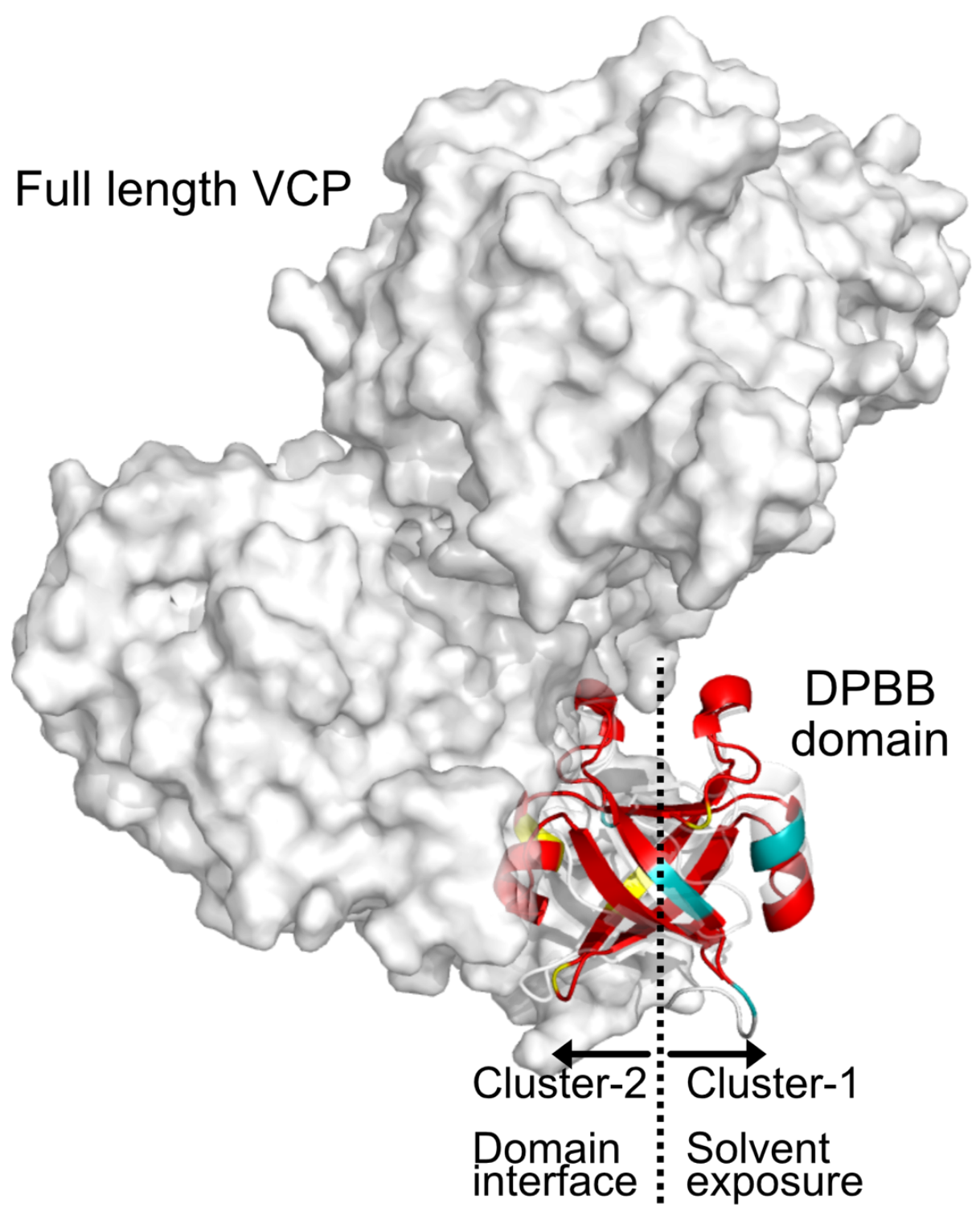

Figure S7. Different environments of symmetrical faces in the full-length VCP protein. The DPBB domain and other domains in the full-length VCP from Thermoplasma acidophilum (PDBID 5G4F) are represented by the white cartoon model and surface model, respectively. The crystal structure of mkDPBB_84 is superimposed with the DPBB domain and colored as in Fig. 1E. Cluster-1 is exposed to the solvent and cluster-2 is an interface to the other domain. 

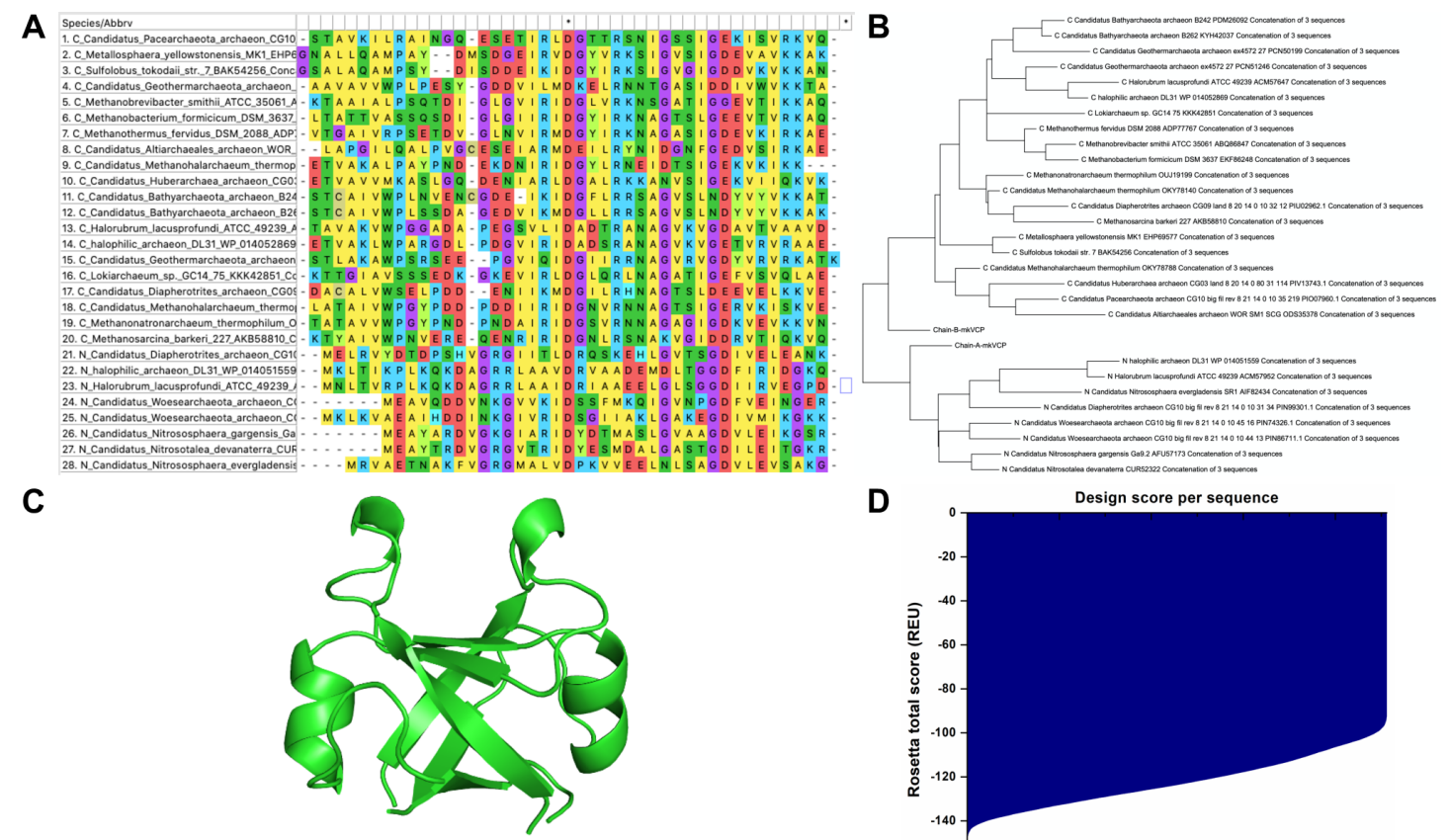

Symmetric DPBB backbone template based on mkVCP

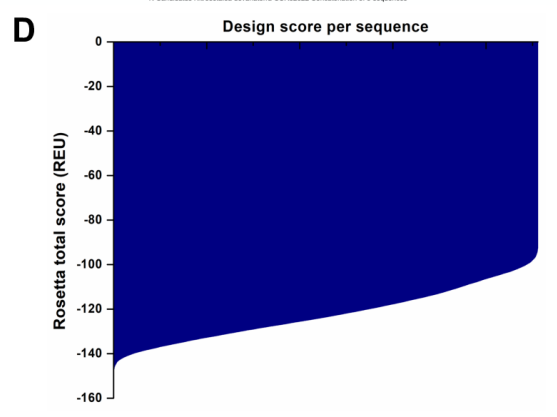

Figure S8. Reverse engineering evolution strategy to design a fully symmetric DPBB domain.

(A) DPBB sequences from different organisms were aligned (B) to produce a phylogenetic tree, for use as input to generate possible ancestral sequences. (C) The ancestral sequences were mapped onto the symmetric DPBB backbone template based on mkVCP_DPBB and (D) scored using pyRosetta. 

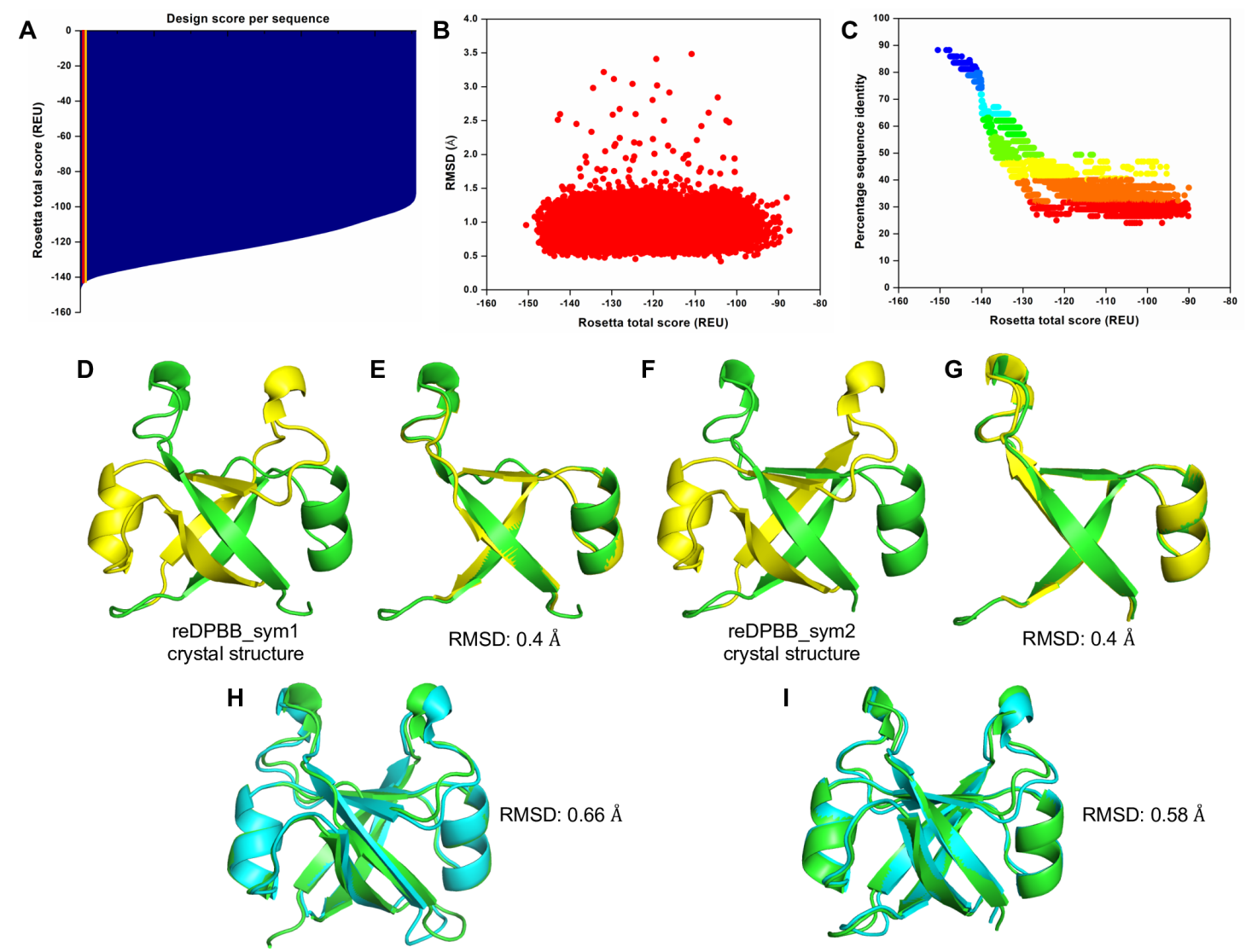

crystal structure

RMSD: $0.4 \AA$

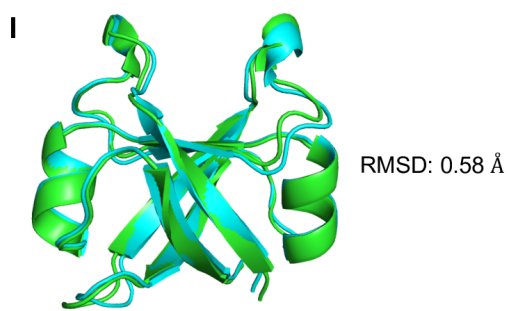

Figure S9. Structural and physicochemical parameters from Rosetta-design calculations, and structural differences between crystal structures and computationally modeled structures of symmetrical DPBBs. (A) Rosetta computed design score per sequence after the ancestral sequences were mapped onto the fully symmetrical DPBB structural model, where the red and yellow lines represent the top-scored reDPBB_sym1 and reDPBB_sym2 designs, respectively. (B) Rosetta scores versus the RMSD of the designs showing well-spread RMSDs from the template. (C) Rosetta total score versus the percentage sequence identity of the designs, which had sequence identities ranging from $25-90 \%$. (D) Crystal structure of reDPBB_sym1. (E) Superimposition and computed RMSD between each half-barrel. (F) Crystal structure of reDPBB_sym2. (G) Superimposition and computed RMSD of each half-barrel, shown in green and blue colors, respectively. Structural superimpositions of $(\mathrm{H})$ the reDPBB_sym1 crystal 
structure (green) with the Rosetta generated model (cyan) and (I) the reDPBB_sym2 crystal structure (green) with the Rosetta generated model (cyan), with RMSDs of $0.66 \AA$ and $0.58 \AA$, respectively. 


\section{A Backrub ensemble, hpatch}

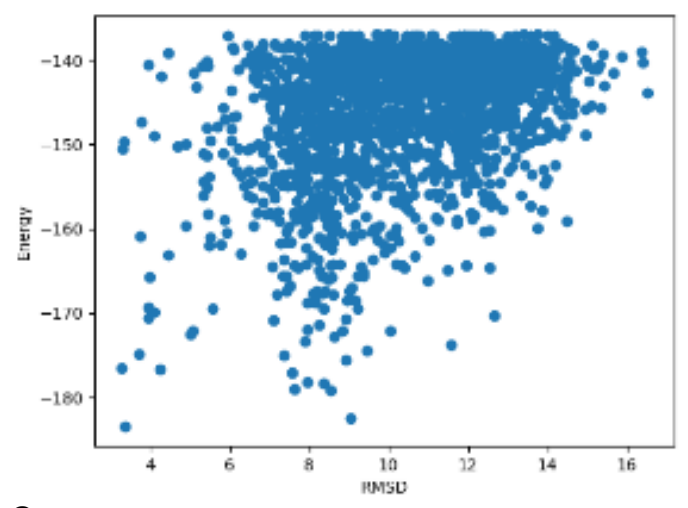

C MD ensemble, hpatch

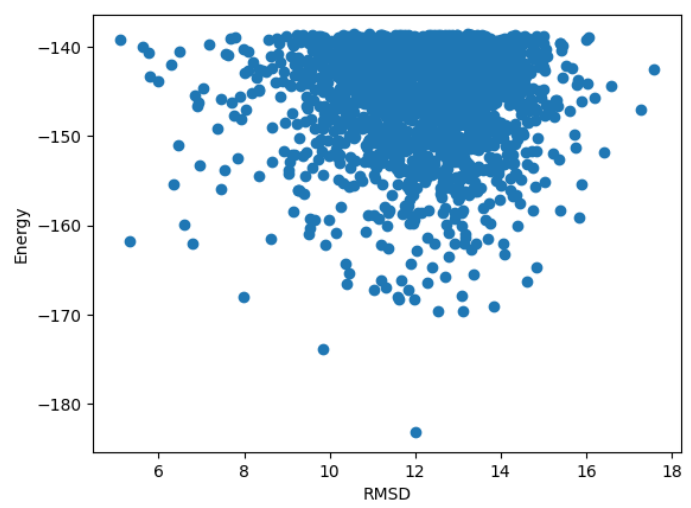

\section{B Backrub ensemble, no hpatch}

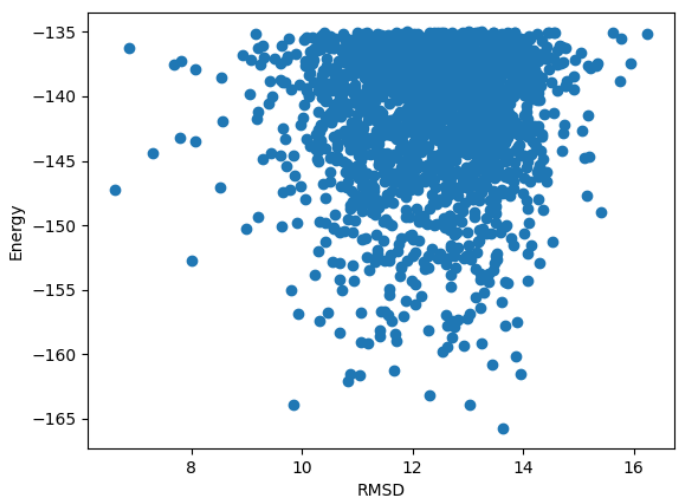

D MD ensemble, no hpatch

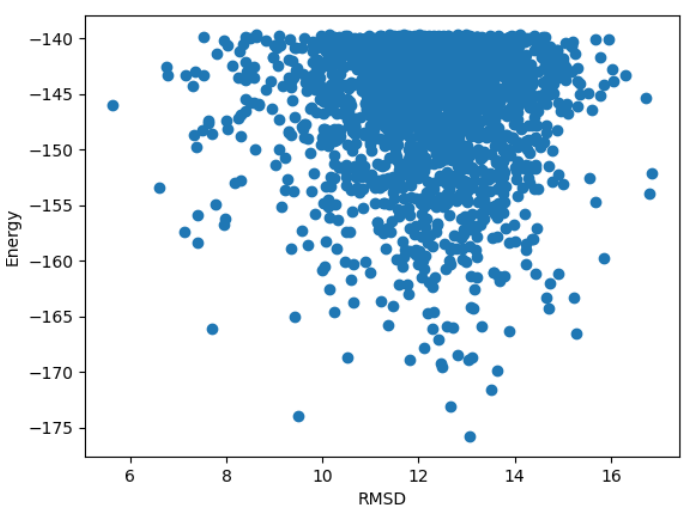

Figure S10. Forward folding profiles of MS designs. For each design, 30,000 protein models were predicted. The RMSD to the template structure was computed for the 1,000 top scoring models. The presence of dots in the bottom left corner of the Backrub ensemble with hpatch plot (A) indicates that EdaRose predicted low energy models within $4 \AA$ from the target structure. For comparison, the Backrub ensemble without hpatch (B) and the MD ensembles with (C) and without (D) hpatch are shown. 


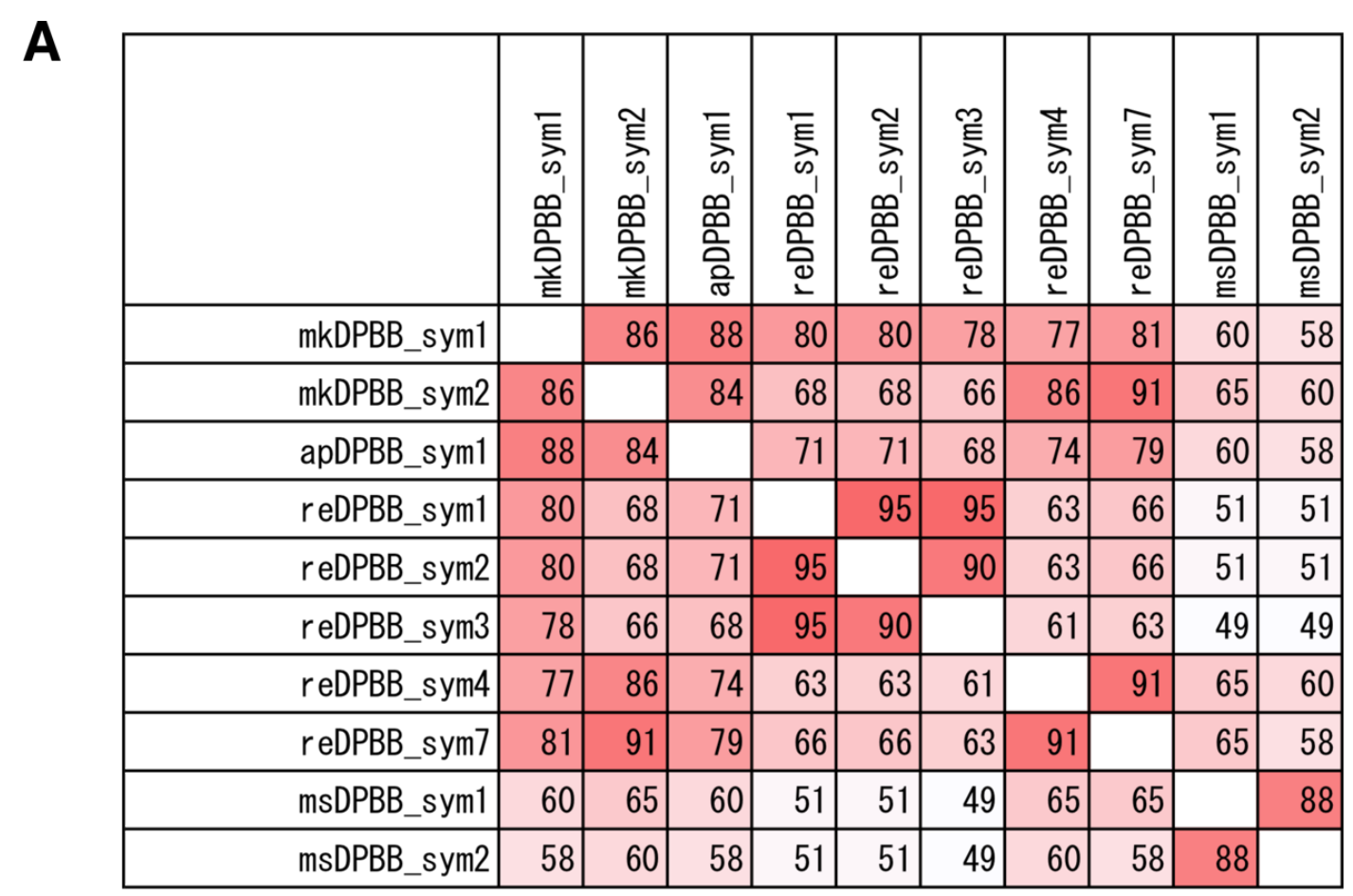

B

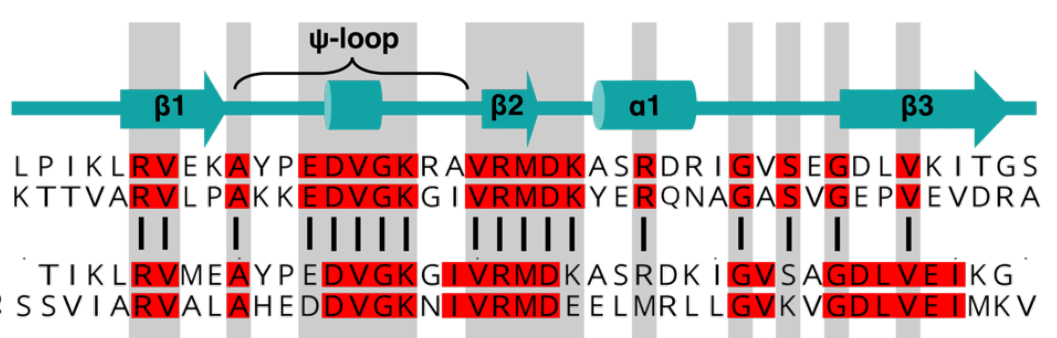

Fig. S11. Sequence diversity in symmetrically designed DPBBs. (A) Sequence identities (\%) between each pair of repeat units in the symmetrically designed DPBBs. msDPBB_sym1 and 2 share only 49\% sequence identity with reDPBB_sym3. (B) Pairwise sequence alignment of the repeat units in reDPBB_sym3 and msDPBB_sym2 along with the starting template, mkVCP_DPBB. The symmetrically-conserved positions in the mkVCP_DPBB are highlighted in gray. Considering that both designs were created from the same starting model and should be biased toward being highly homologous to the original sequence, the potential diversity of the possible DPBB sequences could still be underestimated. If we compare the residues at the non- 
bioRxiv preprint doi: https://doi.org/10.1101/2021.02.22.432383; this version posted February 23, 2021. The copyright holder for this

preprint (which was not certified by peer review) is the author/funder. All rights reserved. No reuse allowed without permission.

symmetric positions in the starting model, then reDPBB_sym3 and msDPBB_sym2 share only $26 \%$ sequence identity. 
A

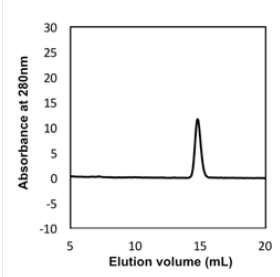

B

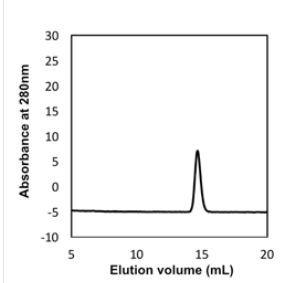

C

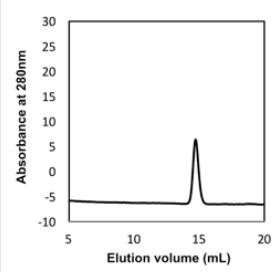

D

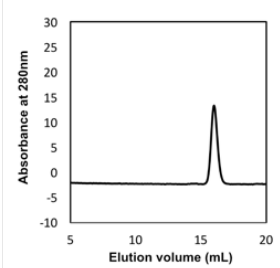

E

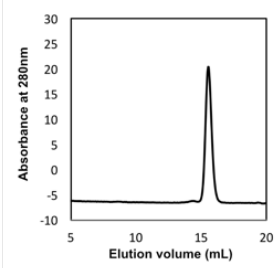

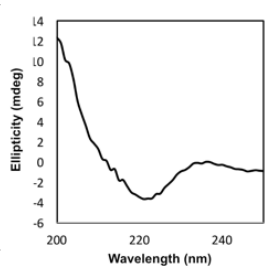
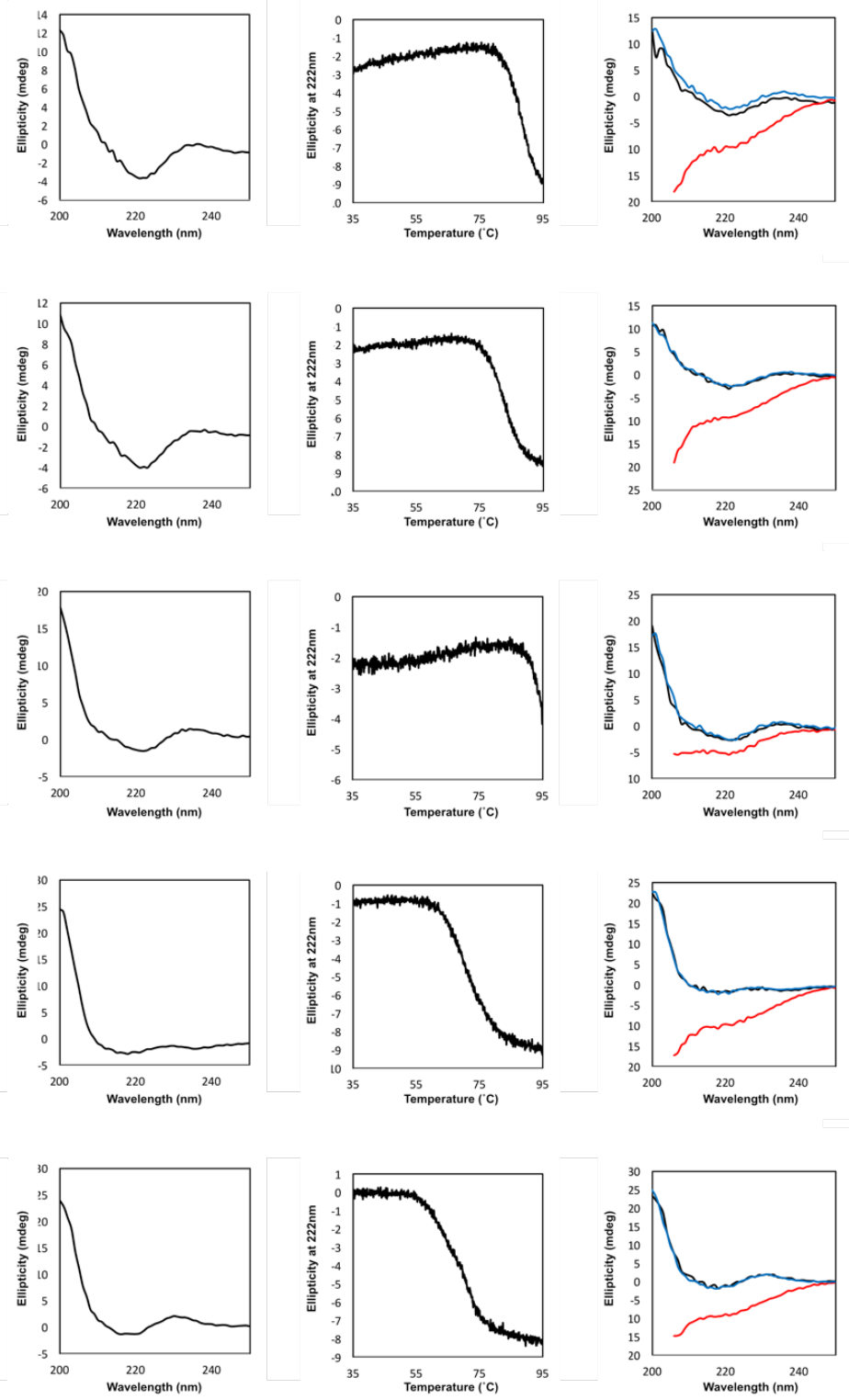

Figure S12. Experimental characterization of symmetrical DPBBs designed by the REdesign method. Size exclusion chromatography, CD spectra, denaturation curves, and comparisons of $\mathrm{CD}$ spectra at different temperatures (black: $35^{\circ} \mathrm{C}$; red: $95^{\circ} \mathrm{C}$; blue (refolding): $95^{\circ} \mathrm{C} \rightarrow 35^{\circ} \mathrm{C}$ ) for (A) reDPBB_sym1, (B) reDPBB_sym2, (C) reDPBB_sym3, (D) reDPBB_sym4, and (E) reDPBB_sym7 are shown in the panels from left to right. 
A

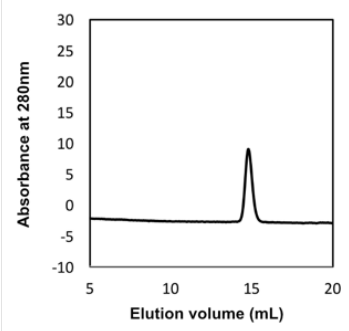

B

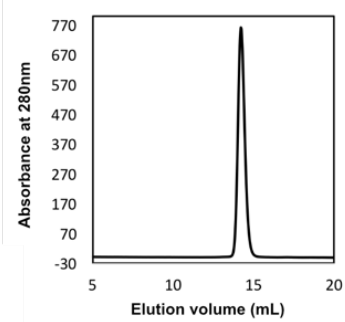

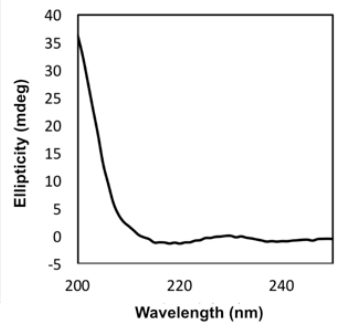
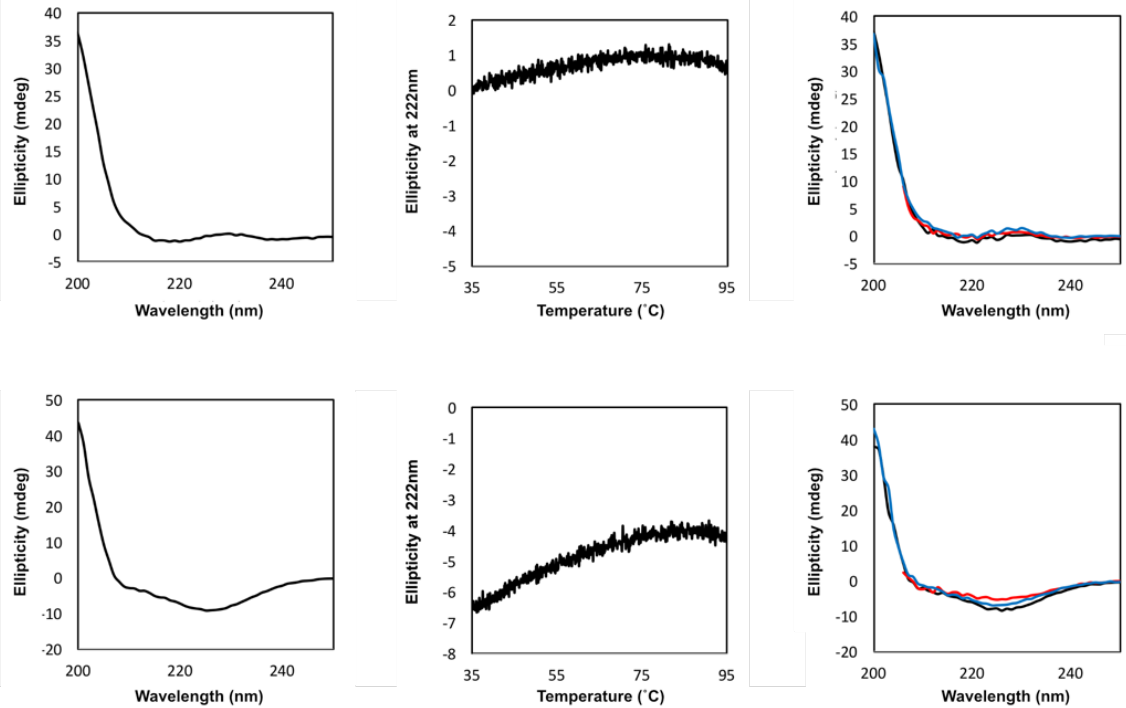
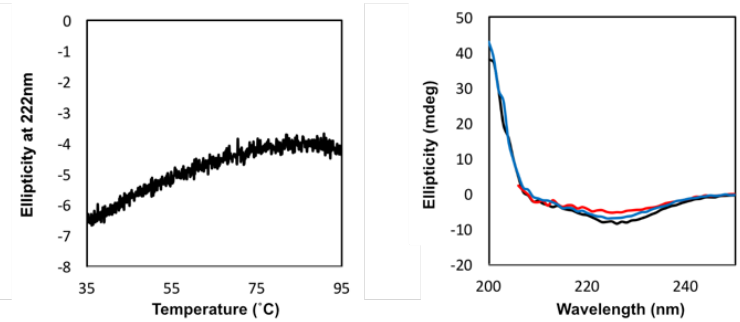

Figure S13. Experimental characterization of symmetrical DPBBs designed by the MSdesign method. Size exclusion chromatography, CD spectra, denaturation curves, and comparisons of $\mathrm{CD}$ spectra at different temperatures (black: $35^{\circ} \mathrm{C}$; red: $95^{\circ} \mathrm{C}$; blue (refolding): $95^{\circ} \mathrm{C} \rightarrow 35^{\circ} \mathrm{C}$ ) for (A) msDPBB_sym1 and (B) msDPBB_sym2 are shown in the panels from left to right. 
A
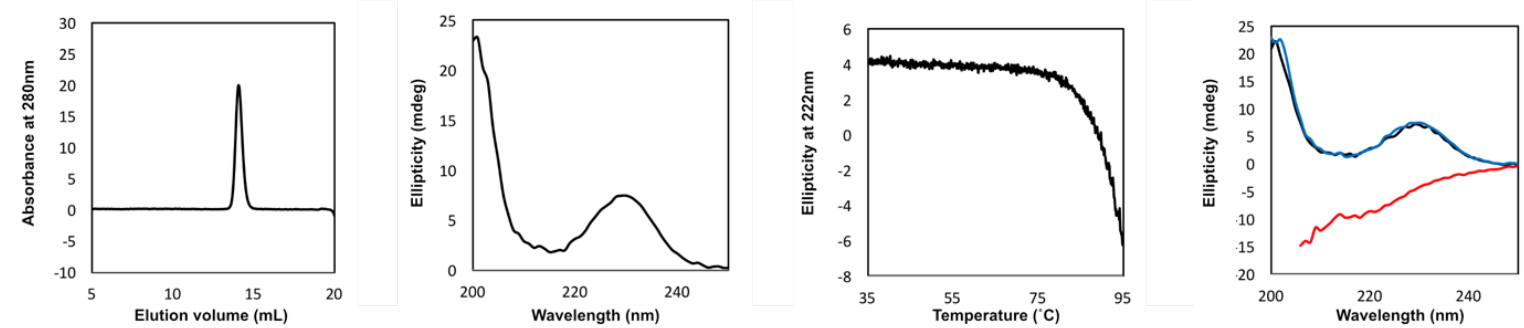

B
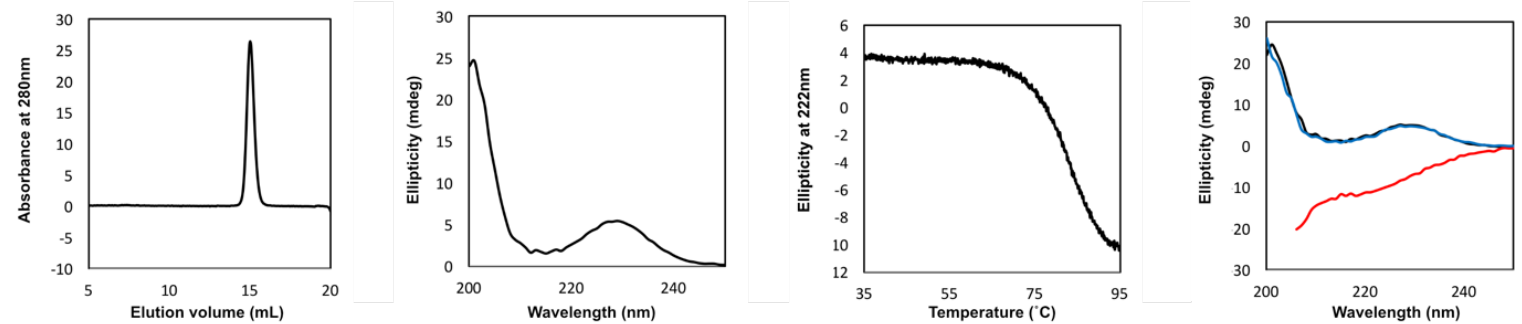

C
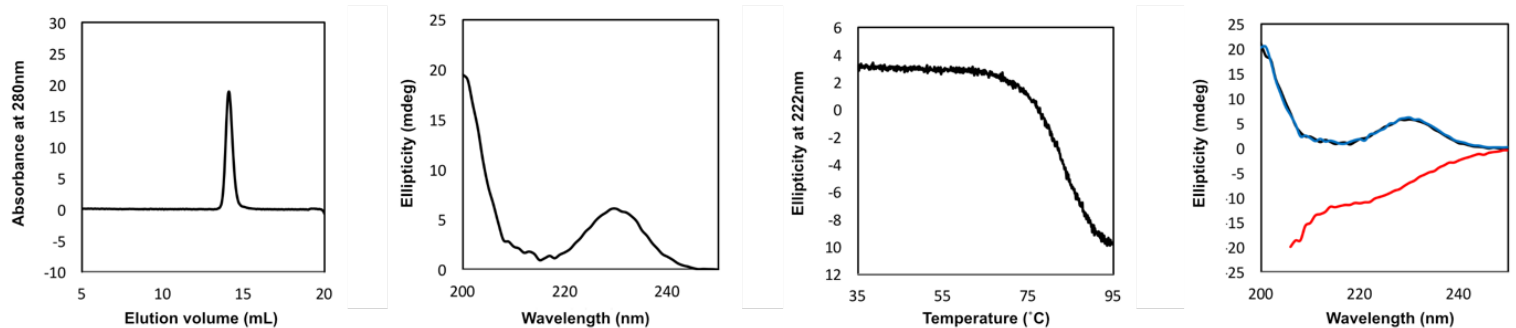

D
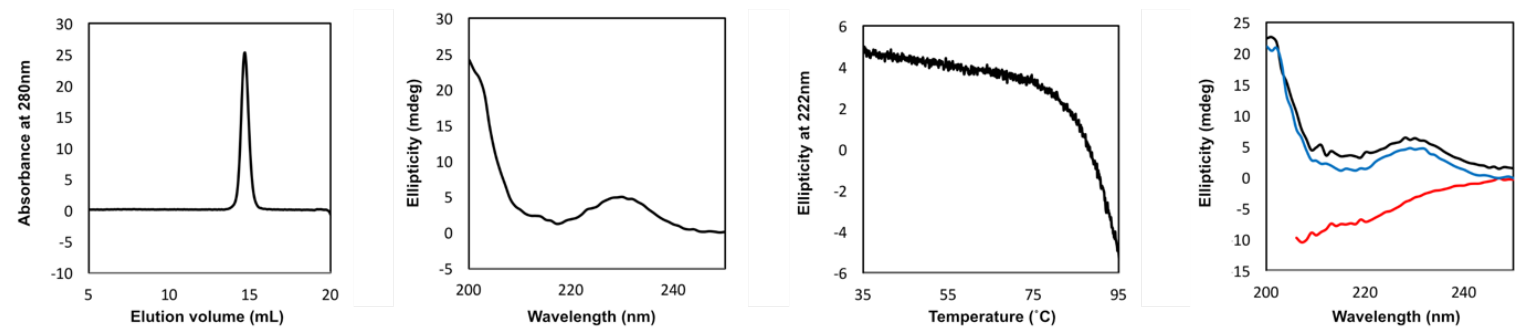

Figure S14. Experimental characterization of the halved fragments. Size exclusion chromatography, CD spectra, denaturation curves, and comparisons of CD spectra at different temperatures (black: $35^{\circ} \mathrm{C}$; red: $95^{\circ} \mathrm{C}$; blue (refolding): $95^{\circ} \mathrm{C} \rightarrow 35^{\circ} \mathrm{C}$ ) for $(\mathrm{A}) \mathrm{mk} 1 \mathrm{~h},(\mathrm{~B}) \mathrm{mk} 2 \mathrm{~h}$, (C) ap1h, and (D) ap2h are shown in the panels from left to right. 

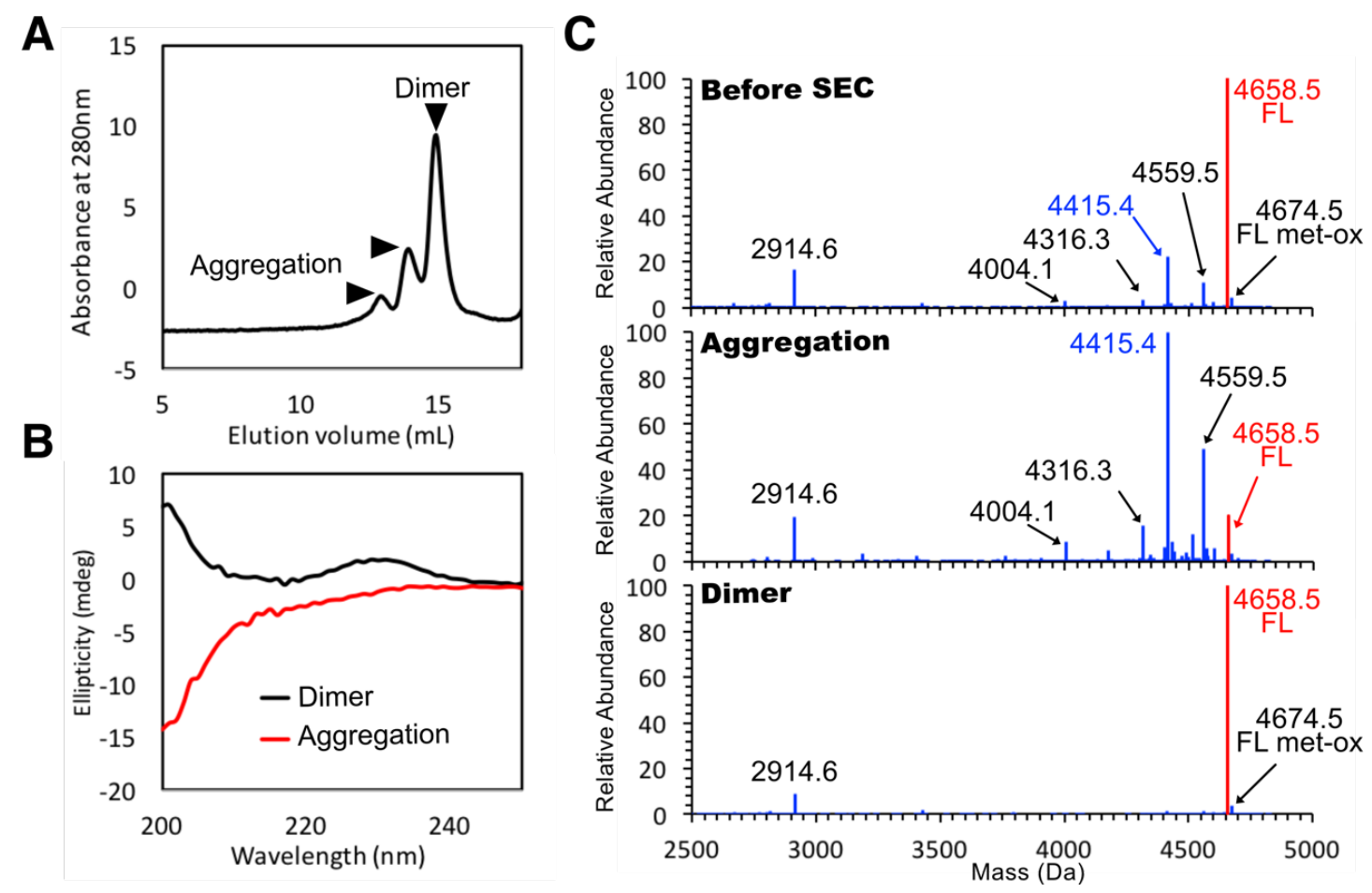

Figure S15. Characterization of the low-purity chemically-synthesized mk1h peptide (75.06\%). (A) SEC analysis showing the aggregated and dimeric states of the dissolved mk1h peptide. (B) CD spectra indicating that the aggregated and dimer species separated in Fig. S12B, adopt random-coil and $\alpha / \beta$ structures, respectively. (C) The peptide species in the sample before and after the SEC purification were analyzed by LC/MS, and the deconvoluted mass spectra are shown. The labels for the full-length mk1h peptide (4658.5 Da) and the major contaminant peptide (4415.4 Da) are highlighted in red and blue, respectively. While most of the contaminant peptides were enriched in the aggregation fraction, the full-length $\mathrm{mk} 1 \mathrm{~h}$ was enriched in the dimer fraction. 
A
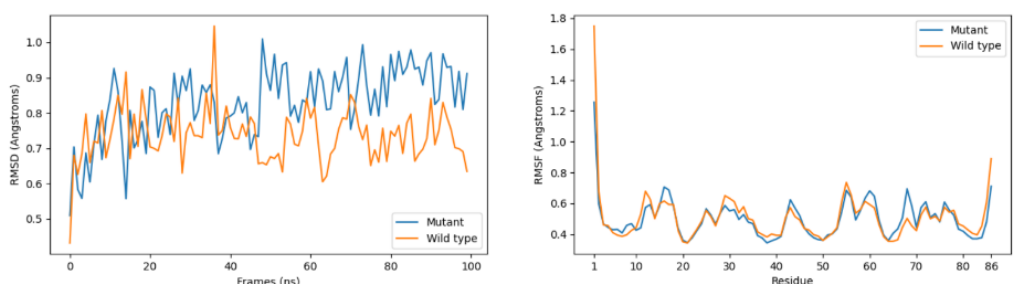

B
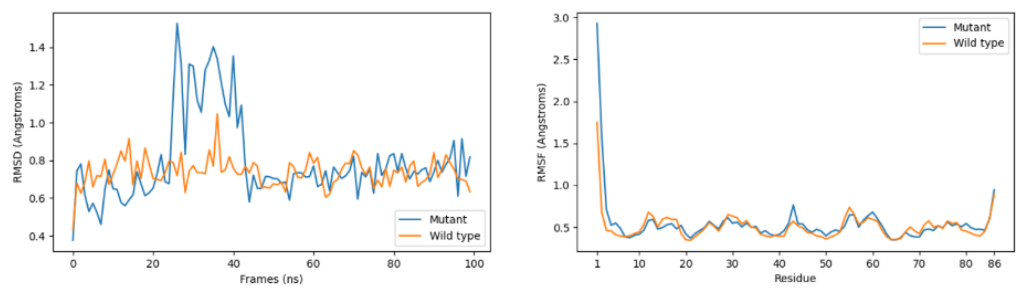

C
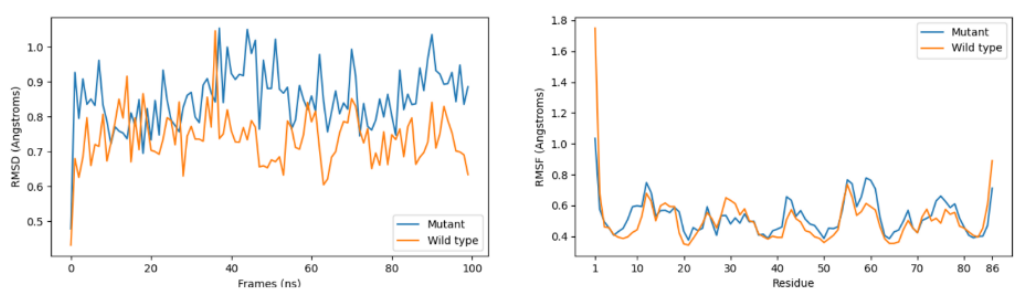

D
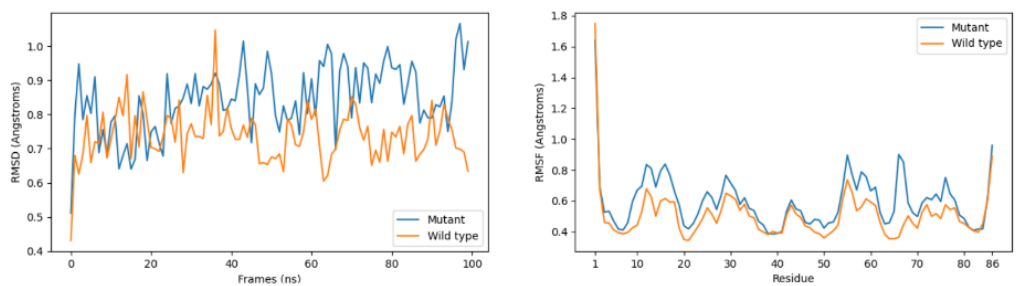

$\mathbf{E}$
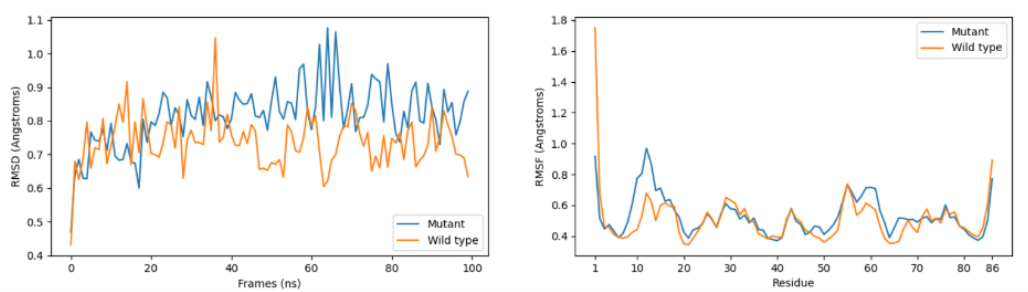

$\mathbf{F}$
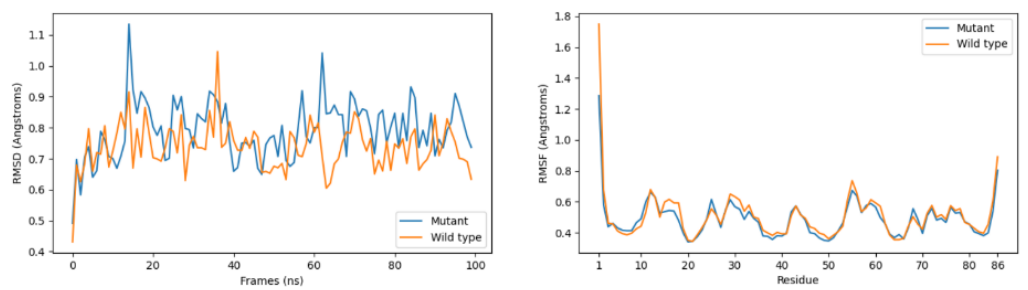

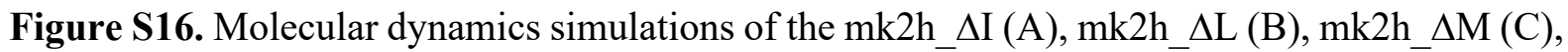

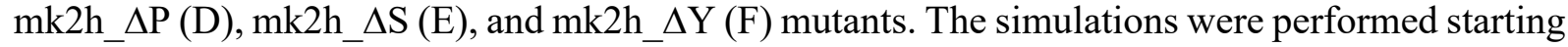


from structural models in the form of the linked repeats with 86 amino acid residues. The backbone RMSD to the reference structure along $100 \mathrm{~ns}$ of simulation is plotted on the left, and the backbone RMSF by residue averaged over $100 \mathrm{~ns}$ of simulation is plotted on the right. No large conformational change, except around the N-terminus, was observed in both the original (mk2h) and mutant designs. 
A

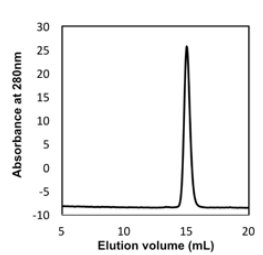

B

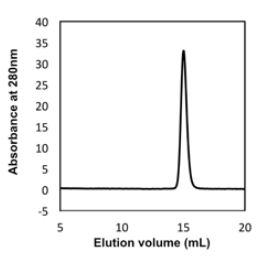

C

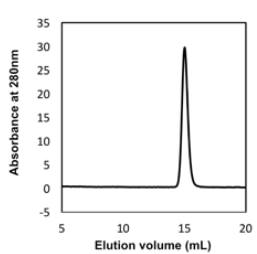

D

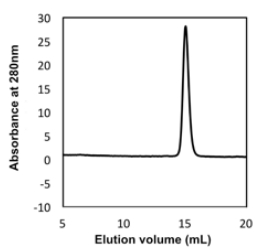

E

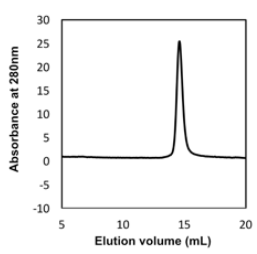

F

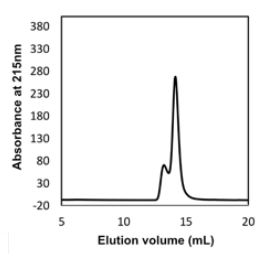

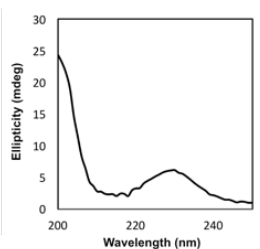
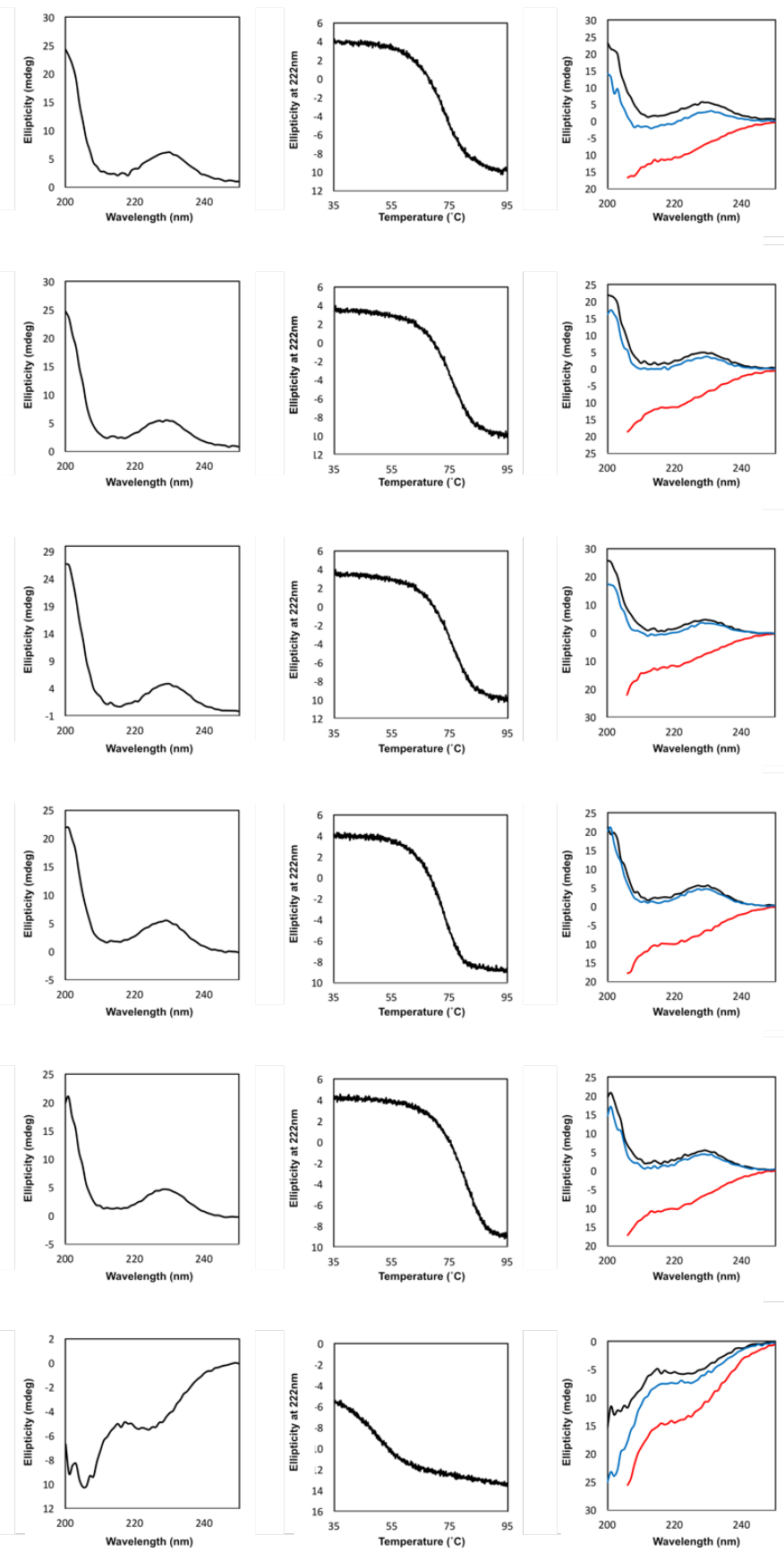

$$
\text { . }
$$




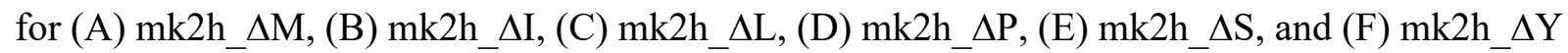
are shown in the panels from left to right. 
A
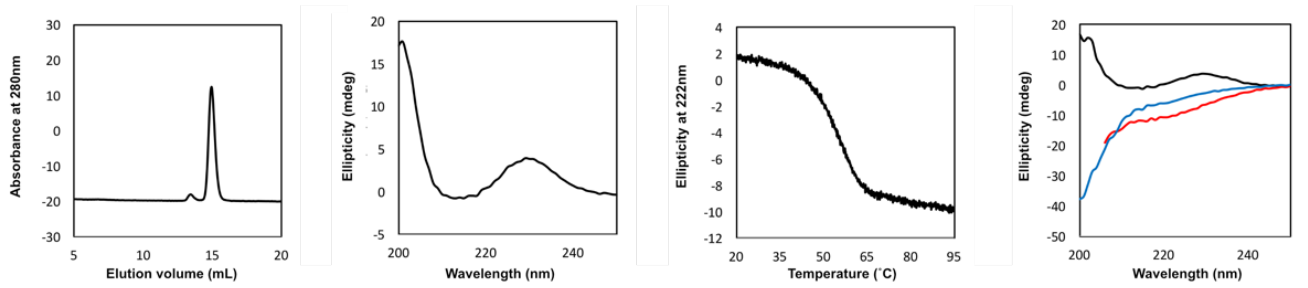

B
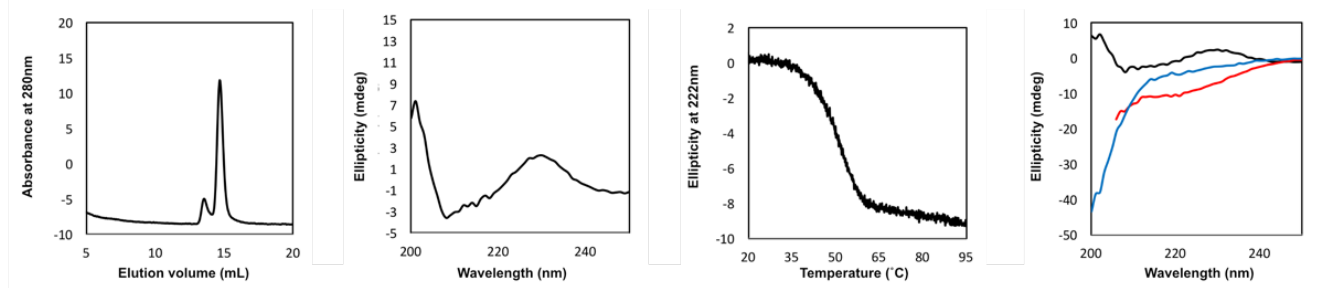

C
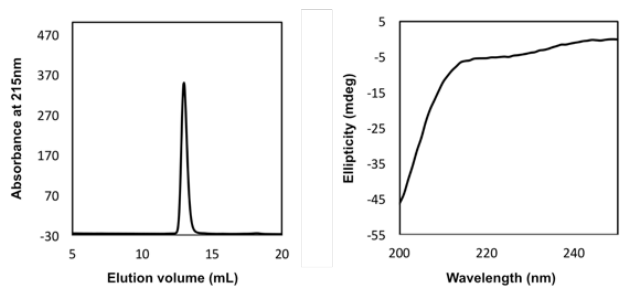

D
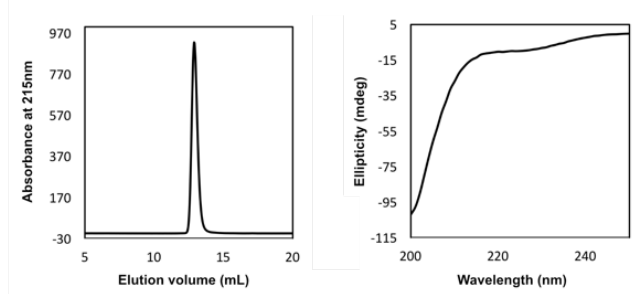

E
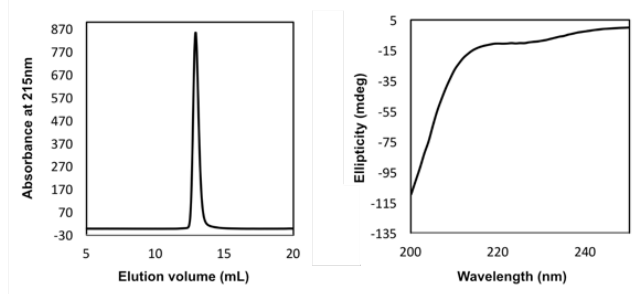

Figure S18. Experimental characterization of $\mathrm{mk2h}$ mutants containing 7, 8, or 10 amino acid repertoires. Size exclusion chromatography, CD spectra, denaturation curves, and comparisons of $\mathrm{CD}$ spectra at different temperatures (black: $10^{\circ} \mathrm{C}$; red: $95^{\circ} \mathrm{C}$; blue (refolding):

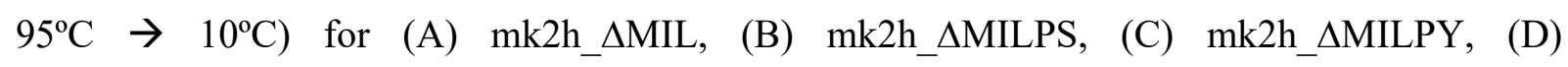
mk2h_AMILSY, and (E) mk2h_AMILPYS are shown in the panels from left to right. 


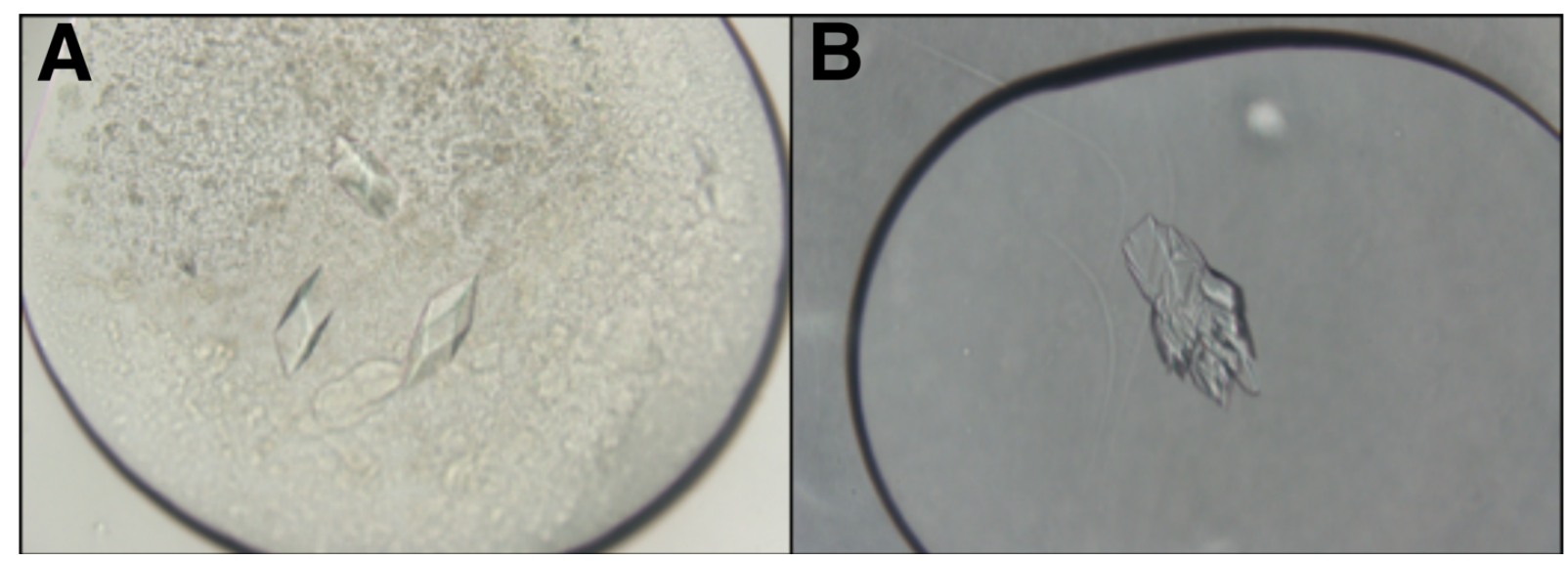

Fig. S19. Crystals of mk2h_AMILPYS. The crystals were obtained under two different conditions containing (A) an undissolved suspension of the chemically-synthesized peptide in 3 M sodium malonate and (B) the dissolved peptide in 2.1 M DL-malic acid, $\mathrm{pH}$ 7.0. The determined crystal structures are shown in Fig. 4D and Fig. S6K, respectively. 
A

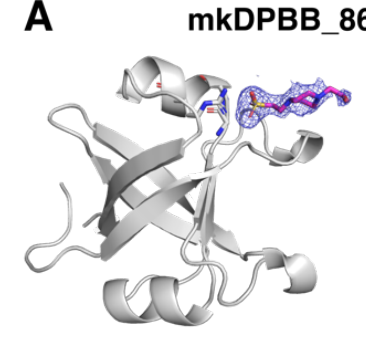

C

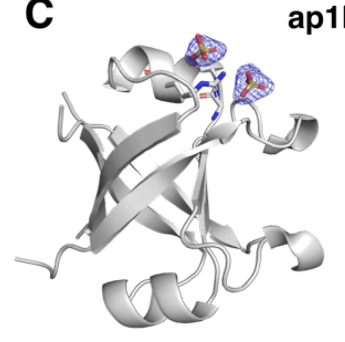

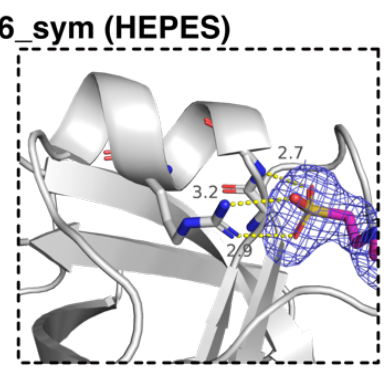

ap1h (sulfate)

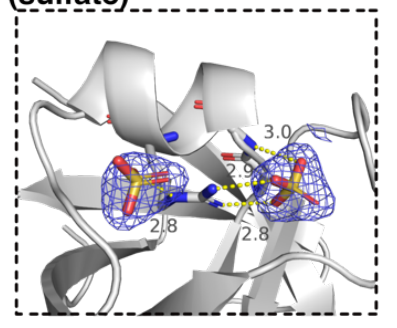

B

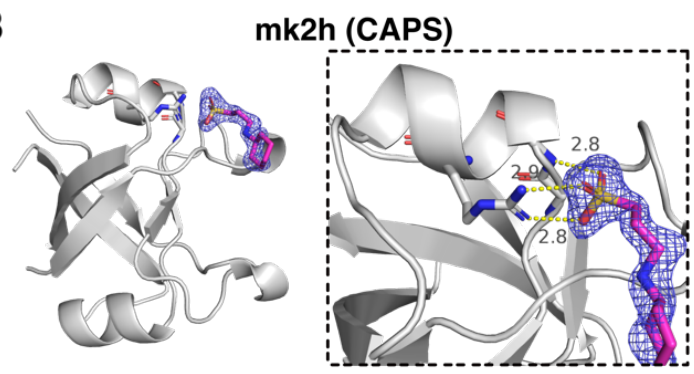

D

mk2h_AMILPS (malonate)

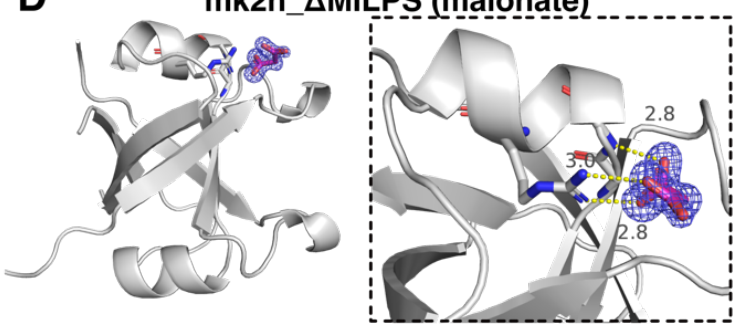

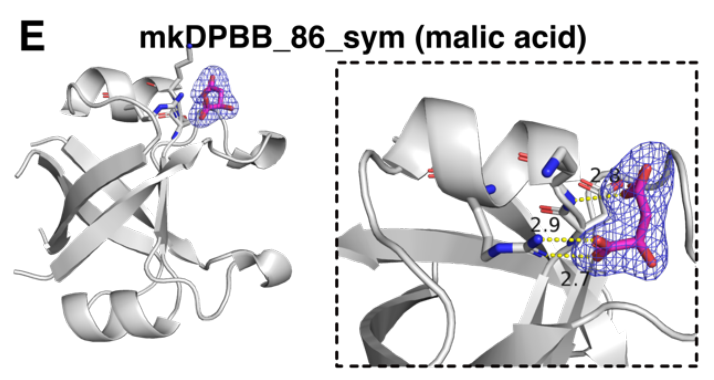

Figure S20. Positively-charged pockets in designed DPBBs occupied by negatively-charged

ligands. (A-E) Crystal structures and close-up views of the conserved positively-charged pockets of designed DPBB domains. (A) mkDPBB_sym_84, (B) mk2h, (C) ap1h, (D) mk2h_AMILPS, and (E) mk2h_SMILPYS interactions with HEPES, CAPS, sulfate ion, malonate ion, and malic acid, respectively, at the positively charged pocket around their $\alpha$-helices. The $\mathrm{N}-\mathrm{H}$ group at the $\mathrm{N}$-terminal peptide bond of the $\alpha 1$ helix and the arginine residue positioned in the middle of the $\alpha 1$ helix (Arg30 in mk2h__MILPYS) form salt-bridges with the negatively-charged ligands. This observation supports the idea that the ancestral DPBB proteins composed of the repeated sequence or halved fragments could have functioned as cofactor- or nucleic acid-binding proteins, like their extant descendants $(43,44)$. 


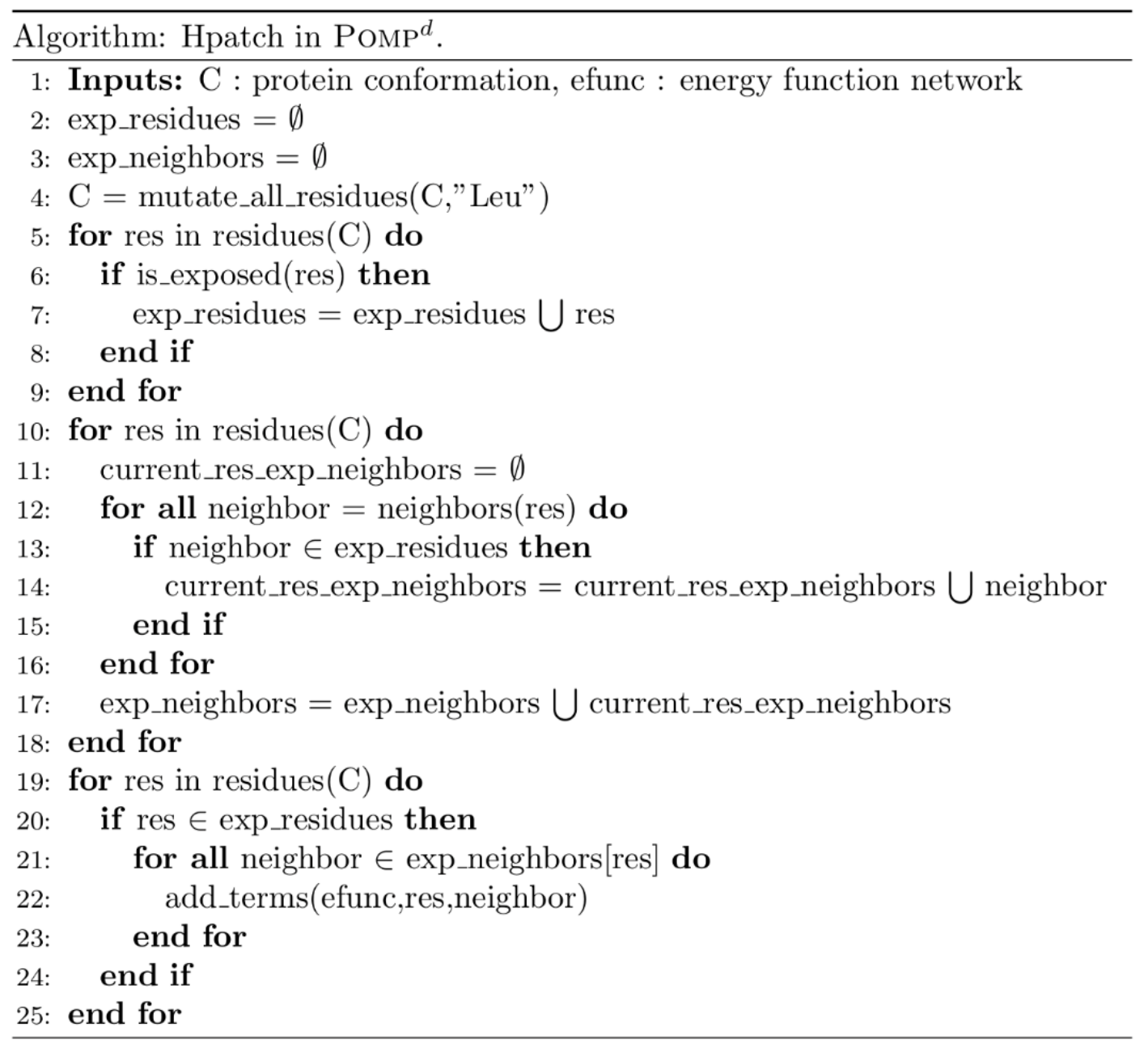

Algorithm S1. Hpatch procedure. Exposed surface residues are calculated with pyRosetta, after mutating the whole protein to LEU in order to ensure the equal treatment of all residue positions. For each residue, the list of all its exposed neighbors is computed. Finally, the neighboring hydrophobic pairs at the surface are forbidden with new energy terms. 


\section{Table S1. Sequences and internal identities of the natural and designed DPBB domains.}

\begin{tabular}{|c|c|c|c|c|c|}
\hline Protein name & aa & pI & $\begin{array}{l}\mathrm{Mw} \\
(\mathrm{kDa})\end{array}$ & Sequence & $\begin{array}{l}\text { Sequence } \\
\text { identity }\end{array}$ \\
\hline \multicolumn{6}{|c|}{ Isolated DPBBs from VCP proteins } \\
\hline \multirow{2}{*}{ taVCP_DPBB } & \multirow{2}{*}{91} & \multirow{2}{*}{9.6} & \multirow{2}{*}{10.2} & MESNNG I I LRVAEANSTDPGMSRVRLDESSRRLLDAE I GDVVEIEKVR & \multirow{2}{*}{$16 / 43(37 \%)$} \\
\hline & & & & KTVGRVYRARPEDENKGIVRIDSVMRNNCGASI GDKVKVRKVR & \\
\hline \multirow{2}{*}{ mkVCP_DPBB } & \multirow{2}{*}{90} & \multirow{2}{*}{9.5} & \multirow{2}{*}{9.8} & MPGLPIKLRVEKAYPEDVGKRAVRMDKASRDRIGVSEGDLVKITGS & \multirow{2}{*}{$18 / 43(42 \%)$} \\
\hline & & & & KTTVARVLPAKKEDVGKGIVRMDKYERQNAGASVGEPVEVDRAE & \\
\hline \multirow{2}{*}{ apVCP_DPBB } & \multirow{2}{*}{91} & \multirow{2}{*}{ 8. 1} & \multirow{2}{*}{9.9} & MANSSVELRVSEAYPRDVGRKIVRIDRQTAARLGVEVGDFVKVSKGD & \multirow{2}{*}{$20 / 44(45 \%)$} \\
\hline & & & & RSVVAVVWPLRPDDEGRGI IRMDGYLRAALGVTVGDTVTVEKAE & \\
\hline \multicolumn{6}{|c|}{ Symmetric DPBBs designed using Symmetric-conservation method (SC-design) } \\
\hline \multirow{2}{*}{ apDPBB_sym_63 } & \multirow{2}{*}{91} & \multirow{2}{*}{9.8} & \multirow{2}{*}{10.0} & MANSSVELRVSEAYPEDVGRKIVRMDKQTRARLGVSVGDFVKVSKGD & \multirow{2}{*}{$28 / 44(63 \%)$} \\
\hline & & & & RSVVARVWPARPEDVGRGIVRMDKYLRAALGVSVGDTVTVEKAE & \\
\hline anDPRB cym 70 & 01 & 00 & 10.1 & MANSSVELRVLEARPEDVGRKIVRMDKQTRARLGVSVGDYVEVKKVD & $25 \% 140(70)$ \\
\hline apuPBB_sym_19 & प्रा & 9.8 & 10.1 & RSVVARVLPARPEDVGRGIVRMDKYLRAALGVSVGDYVEVKKVE & $35 / 44 \quad(/ 9 \%)$ \\
\hline ORPPD & 01 & 0 & 101 & MANSSVELRVAEAYPEDVGRKIVRMDKQTRAKLGVSVGDYVEVKKVD & $27(14$ (0100) \\
\hline apUPBB_sym_84 & 91 & 9. 2 & 10.1 & RSVVARVAEAYPEDVGRGIVRMDKYLRAKLGVSVGDYVEVKKVE & $3 / / 44(84 \%)$ \\
\hline$M$ DPRP 67 & 00 & 00 & 07 & MPGLSVKLRVEKAYPEDVGKRIVRMDKASRARLGVSVGDLVKVTKS & $00: 12-(c 70)$ \\
\hline IIIRUTDD_Syill_oI & 90 & 9.0 & 9.1 & KSVVARVLPAKPEDVGKGIVRMDKYERANLGVSVGDPVEVDKAE & $29 / 40(0 / 70)$ \\
\hline 01 & 00 & 10.1 & $0=0$ & MPGLSVKLRVLKARPEDVGKRIVRMDKASRARLGVSVGDYVEVKKV & 25102010 \\
\hline |mKLPDD_SyII_oI & 90 & 10.1 & 9.9 & KSVVARVLPARPEDVGKGIVRMDKYERANLGVSVGDYVEVKKVE & $35 / 43(81 \%)$ \\
\hline ro & 00 & $0=5$ & 0 & MPGLSVKLRVAEAYPEDVGKRIVRMDKASRAKLGVSVGDYVEVKKV & (0) \\
\hline ImKUPBB_sym_80 & 90 & 9.5 & 9.9 & KSVVARVAEAYPEDVGKGIVRMDKYERAKLGVSVGDYVEVKKVE & $3 / / 43 \quad(86 \%)$ \\
\hline MPRP 1 & 00 & 07 & $0=6$ & MPGLSVKLRVAEAYPEDVGKGIVRMDKASRAKLGVSVGDYVEVKKV & $12 / 12$ \\
\hline |miKUPDD_symI & 89 & 9.1 & 9.0 & LSVKLRVAEAYPEDVGKGIVRMDKASRAKLGVSVGDYVEVKKV & $43 / 43(100 \%)$ \\
\hline MPDPP & 80 & 06 & 0 & MPGKSVVARVAEAYPEDVGKRIVRMDKYERAKLGVSVGDYVEVKKV & $12 / 12=10000$ \\
\hline ImKDPBB_symL & 89 & 9.6 & 9.9 & KSVVARVAEAYPEDVGKRIVRMDKYERAKLGVSVGDYVEVKKV & $4 J / 40 \quad(100 \%)$ \\
\hline 1 & 01 & 62 & 100 & MANSSVELRVAEAYPEDVGRGIVRMDKQTRAKLGVSVGDYVEVKKVD & 14 us 11000 \\
\hline apurDb_symI & प्रा & 0.3 & 10.0 & SSVELRVAEAYPEDVGRGIVRMDKQTRAKLGVSVGDYVEVKKVD & $44 / 44(100 \%)$ \\
\hline OPDP & 01 & 07 & 102 & MANRSVVARVAEAYPEDVGRKIVRMDKYLRAKLGVSVGDYVEVKKVE & 14 ( \\
\hline apUPBB_symz & पi & 9.1 & 10.3 & RSVVARVAEAYPEDVGRKIVRMDKYLRAKLGVSVGDYVEVKKVE & $44 / 44(100 \%)$ \\
\hline Symmetric DPBBs desi & $g r$ & se evc & ution & angineering method (RE-design) & \\
\hline 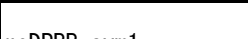 & 05 & 0 & $0=0$ & PIKLRVMEAYPEDVGKGIVRMDKASRDKLGVSAGDLVEIKGSKT & 01 (1) 11000 \\
\hline |reuPBB_symI & 85 & 9.3 & 9.2 & PIKLRVMEAYPEDVGKGIVRMDKASRDKLGVSAGDLVEIKG & $41 / 41 \quad(100 \%)$ \\
\hline ת & 05 & $0=0$ & 0 & PMKLRVMEAYPEDVGKGIVRMDKASREKLGVSAGDLVEIKGSKT & $11 / 11$ \\
\hline redPBD_symL & 85 & 9.3 & 9.2 & PMKLRVMEAYPEDVGKGIVRMDKASREKLGVSAGDLVEIKG & $41 / 41(100 \%)$ \\
\hline YOPDPR & 85 & 0 & 0 & TIKLRVMEAYPEDVGKGIVRMDKASRDKI GVSAGDLVEIKGSKT & $11 / 11$ \\
\hline TredrDD_Syilis & 00 & 9.0 & 9.2 & TIKLRVMEAYPEDVGKGIVRMDKASRDKI GVSAGDLVEIKG & $41 / 41 \quad(100 \%)$ \\
\hline I DPPD & 00 & 0 & 0 & MPGKSVVARVAPAHPEDVGKGIVRMDKYERQNLGVSVGDYVEVKKA & $12 / 12$ \\
\hline redPDD_sym4 & 89 & 9.4 & 9.1 & KSVVARVAPAHPEDVGKGIVRMDKYERQNLGVSVGDYVEVKKA & $43 / 43 \quad(100 \%)$ \\
\hline L & 00 & 60 & $0=6$ & MPGKSVVARVAPAYPEDVGKGIVRMDKYERANLGVSVGDYVEVDKA & $(1000)$ \\
\hline |reUPBB_sym5 & 89 & 0.2 & 9.0 & KSVVARVAPAYPEDVGKGIVRMDKYERANLGVSVGDYVEVDKA & $43 / 43(100 \%)$ \\
\hline C & 00 & 00 & 0.7 & MPGKTVVARVLPAYPEDVGKGIVRMDKYERAKLGVSVGDYVEVEKA & $12 / 12$ \\
\hline |reuPBB_symo & 89 & 8. 8 & 9. 1 & KTVVARVLPAYPEDVGKGIVRMDKYERAKLGVSVGDYVEVEKA & $43 / 43 \quad(100 \%)$ \\
\hline 7 & 00 & 0 & 06 & MPGKSVVARVAPAYPEDVGKGIVRMDKYERAKLGVSVGDYVEVEKA & $12 / 12$ \\
\hline TedrDD_syIIII & 89 & 0.0 & 9.0 & KSVVARVAPAYPEDVGKGIVRMDKYERAKLGVSVGDYVEVEKA & $40 / 40 \quad(100 \%$ \\
\hline Symmetric DPBBs desi & IIIL & -stat & design & method (MS-design) & \\
\hline MDPPR 1 & 86 & 67 & 06 & SSVVARVALAHEDDVGKNIVRMDEDLMRKLGVKVGDYVEIMKK & $112 \quad(1000)$ \\
\hline IIISUPDD_SyIIII & 00 & 0.1 & 9.0 & SSVVARVALAHEDDVGKNIVRMDEDLMRKLGVKVGDYVEIMKK & $4 J / 40 \quad(100 \%)$ \\
\hline$m a D P R R$ & 86 & 51 & 0.5 & SSVIARVALAHEDDVGKNIVRMDEELMRLLGVKVGDLVEIMKV & () \\
\hline IIISUPDD_SyIIIL & 80 & 0.1 & 9.5 & SSVIARVALAHEDDVGKNIVRMDEELMRLLGVKVGDLVEIMKV & 4 \\
\hline
\end{tabular}




\begin{tabular}{|c|c|c|c|c|c|}
\hline \multicolumn{6}{|c|}{ Half fragmented DPBBs } \\
\hline mk1h & 46 & 9.5 & 4. 9 & MPGLSVKLRVAEAYPEDVGKGIVRMDKASRAKLGVSVGDYVEVKKV & - \\
\hline mk2h & 46 & 9.5 & 5.1 & MPGKSVVARVAEAYPEDVGKRIVRMDKYERAKLGVSVGDYVEVKKV & - \\
\hline ap 1h & 47 & 6.1 & 5.2 & MANSSVELRVAEAYPEDVGRGIVRMDKOTRAKLGVSVGDYVEVKKVD & - \\
\hline ap2h & 47 & 9.5 & 5.3 & MANRSVVARVAEAYPEDVGRKIVRMDKYLRAKLGVSVGDYVEVKKVE & - \\
\hline \multicolumn{6}{|c|}{ Simp/ified mk2h } \\
\hline$m k 2 h \_\Delta M$ & 46 & 9.5 & 5.1 & MPGKSVVARVAEAYPEDVGKRIVRVDKYERAKLGVSVGDYVEVKKV & - \\
\hline mk2h_dI & 46 & 9.5 & 5.1 & MPGKSVVARVAEAYPEDVGKRVVRMDKYERAKLGVSVGDYVEVKKV & - \\
\hline$m k 2 h \_\Delta L$ & 46 & 9.5 & 5.1 & MPGKSVVARVAEAYPEDVGKRIVRMDKYERAKVGVSVGDYVEVKKV & - \\
\hline$m k 2 h \_\Delta P$ & 46 & 9.5 & 5.1 & MPGKSVVARVAEAYAEDVGKRIVRMDKYERAKLGVSVGDYVEVKKV & - \\
\hline$m k 2 h \_\Delta S$ & 46 & 9.8 & 5.2 & MPGKKVVARVAEAYPEDVGKRIVRMDKYERAKLGVKVGDYVEVKKV & - \\
\hline$m k 2 h \_\Delta Y$ & 46 & 10. 0 & 5.0 & MPGKSVVARVAEARPEDVGKRIVRMDKAERAKLGVSVGDVVEVKKV & - \\
\hline mk2h_DMIL & 46 & 9.5 & 5.1 & MPGKSVVARVAEAYPEDVGKRVVRVDKYERAKVGVSVGDYVEVKKV & - \\
\hline mk2h_DMILPS & 46 & 9.8 & 5.1 & MPGKKVVARVAEAYAEDVGKRVVRVDKYERAKVGVKVGDYVEVKKV & - \\
\hline mk2h_DMILPY & 46 & 10. 0 & 4. 9 & MPGKSVVARVAEARAEDVGKRVVRVDKAERAKVGVSVGDVVEVKKV & - \\
\hline mk2h_AMILYS & 46 & 10. 2 & 5.0 & MPGKKVVARVAEARPEDVGKRVVRVDKAERAKVGVKVGDVVEVKKV & - \\
\hline mk2h_DMILPYS & 46 & 10. 2 & 5.0 & MPGKKVVARVAEARAEDVGKRVVRVDKAERAKVGVKVGDVVEVKKV & - \\
\hline
\end{tabular}




\begin{tabular}{|c|c|c|c|c|c|c|c|c|c|c|}
\hline 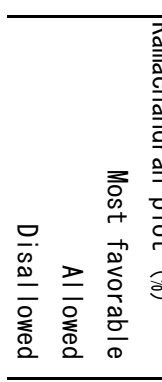 & 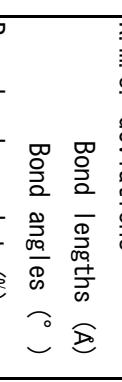 & 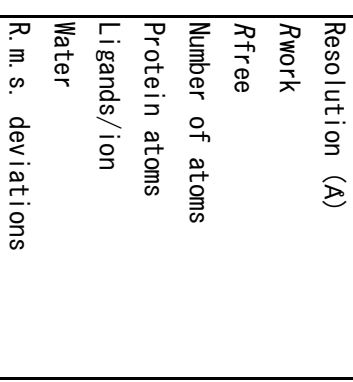 & 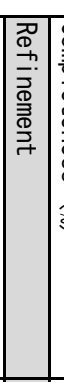 & 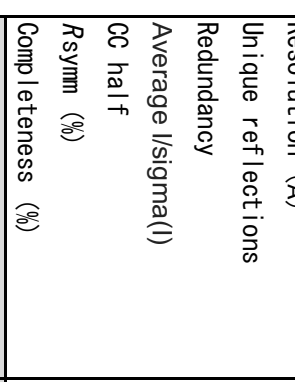 & 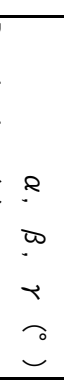 & 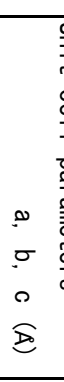 & 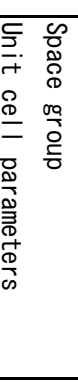 & 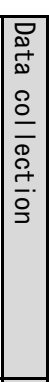 & 呴 & 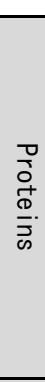 \\
\hline $\begin{array}{lll} & N & 0 \\
0 & \stackrel{\omega}{\omega} & 8\end{array}$ & \begin{tabular}{ll}
0 & 0 \\
$\stackrel{8}{*}$ & 0 \\
\hdashline
\end{tabular} & 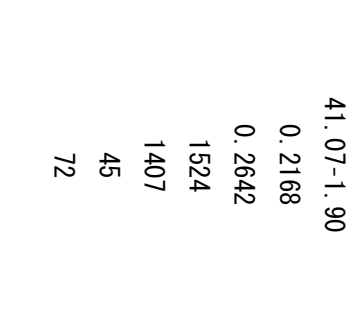 & & 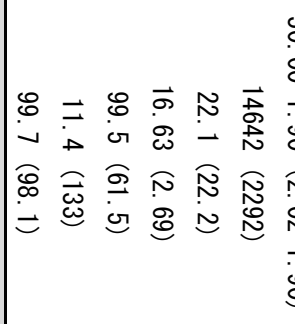 & $\begin{array}{l}8 \\
8 \\
\overrightarrow{0}\end{array}$ & $\begin{array}{l}\overrightarrow{0} \\
0 \\
\overrightarrow{0} \\
0 \\
0 \\
\infty \\
0 \\
0\end{array}$ & $\begin{array}{l}D \\
\omega \\
N\end{array}$ & & 吉 & 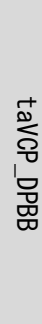 \\
\hline $00 \overrightarrow{8}$ & $\begin{array}{l}\overrightarrow{\hat{N}} 0 \\
\text { 起 }\end{array}$ & 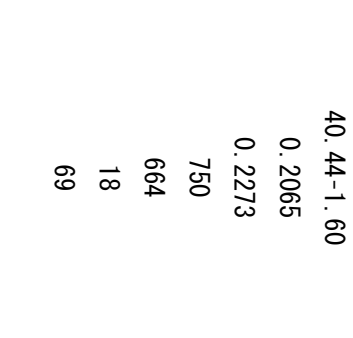 & & 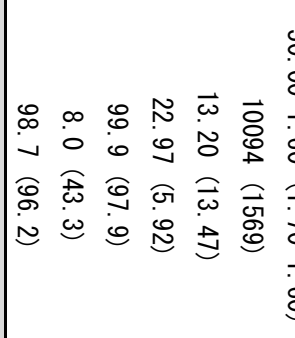 & 8 & 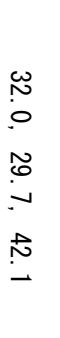 & $\stackrel{\widetilde{N}}{\cong}$ & & 总 & 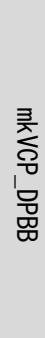 \\
\hline $00 \overrightarrow{8}$ & 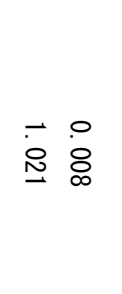 & 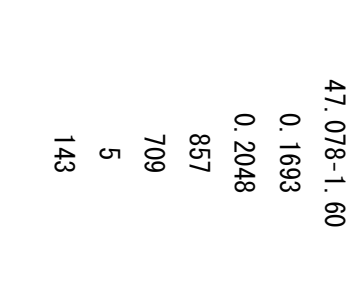 & & 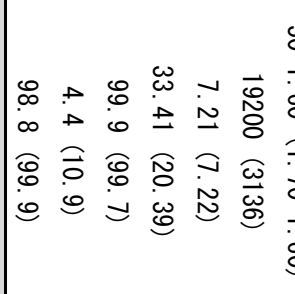 & 8 & $\begin{array}{l}\tilde{\sigma} \\
0 \\
\omega \\
0 \\
0 \\
0 \\
0 \\
0\end{array}$ & 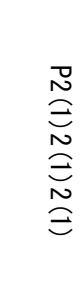 & & 㤩 & 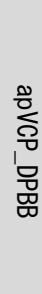 \\
\hline 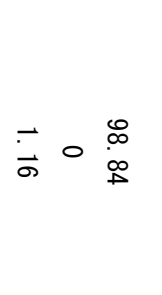 & $\begin{array}{ll}0 & 0 \\
00 & 8 \\
\end{array}$ & 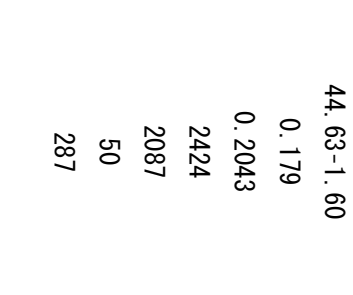 & & 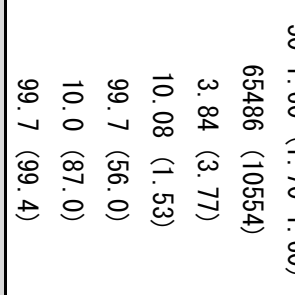 & $\begin{array}{l}8 \\
8 \\
8\end{array}$ & $\begin{array}{l}\omega \\
0 \\
\omega \\
\vec{j} \\
0 \\
\overrightarrow{0} \\
\infty \\
0\end{array}$ & 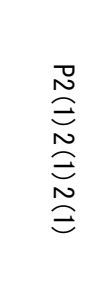 & & 형 & 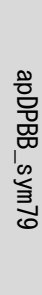 \\
\hline 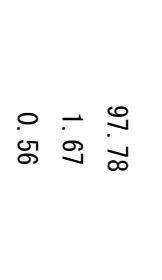 & $\begin{array}{ll}\overrightarrow{0} & 0 \\
0 & 0 \\
\infty & 8\end{array}$ & 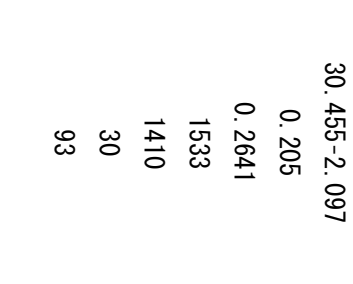 & & 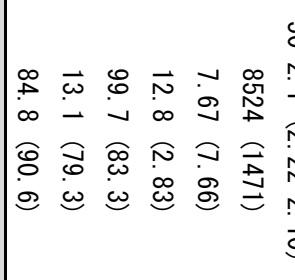 & 8 & $\begin{array}{l}\omega \\
\omega \\
\omega \\
\infty \\
\omega \\
+ \\
\stackrel{\omega}{\omega} \\
\stackrel{\sim}{v}\end{array}$ & $\stackrel{\widetilde{N}}{\cong}$ & & 总 & 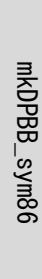 \\
\hline
\end{tabular}




\begin{tabular}{|c|c|c|c|c|c|c|}
\hline $\begin{array}{ll}\vec{r} & \infty \\
0 & \infty \\
\overrightarrow{0} & \infty\end{array}$ & $\begin{array}{l}\overrightarrow{0} \\
\dot{0} \\
\dot{8}\end{array}$ & 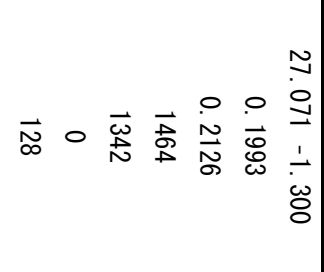 & 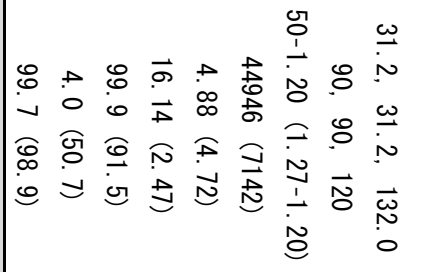 & $\stackrel{\breve{\omega}}{\underline{.}}$ & $\Xi$ & $\begin{array}{l}\text { 콩 } \\
\text { 离 } \\
\text { 怘 } \\
\text { 邑 }\end{array}$ \\
\hline $0 \begin{array}{ll}\overrightarrow{\vec{b}} & \stackrel{\infty}{\infty} \\
\overrightarrow{0} & \stackrel{\infty}{G}\end{array}$ & $\overrightarrow{0}$ & 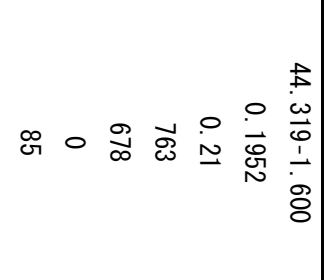 & 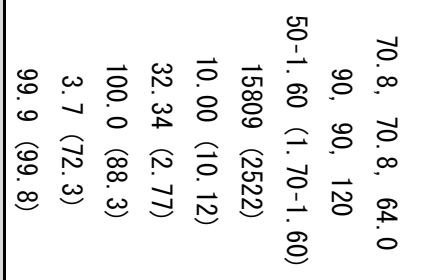 & 己 & ప্টু & 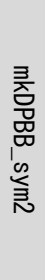 \\
\hline $0 \begin{array}{ll}\overrightarrow{0} & \infty \\
0 & 8 \\
8\end{array}$ & 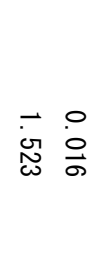 & 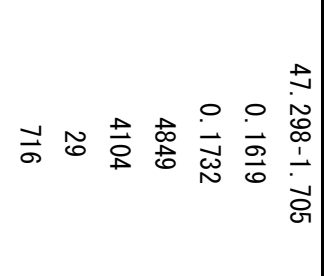 & 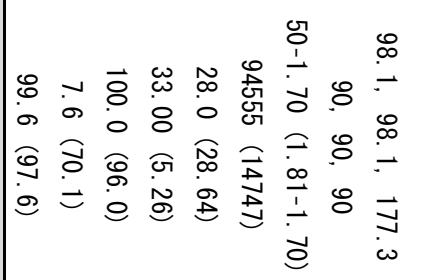 & 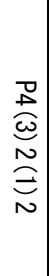 & ప్ర & 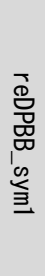 \\
\hline $0 \vec{\sim} \begin{array}{c}\infty \\
\infty\end{array}$ & 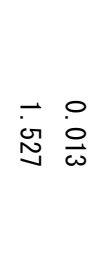 & 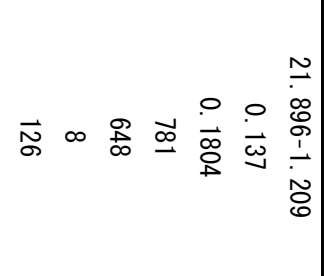 & 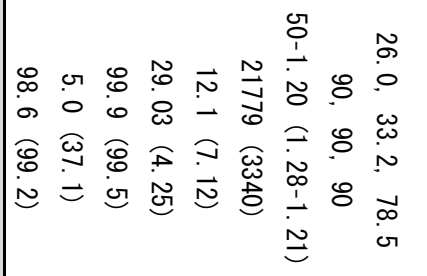 & 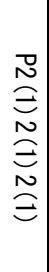 & $\overrightarrow{⿱ ⺌}$ & $\begin{array}{l}\text { 勇 } \\
\text { 离 } \\
\text { 禹 } \\
\text { 言 }\end{array}$ \\
\hline 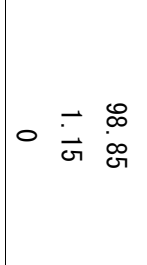 & $\begin{array}{ll}\overrightarrow{\tilde{N}} & 0 \\
\stackrel{0}{N} & \end{array}$ & 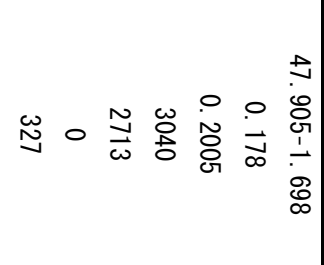 & 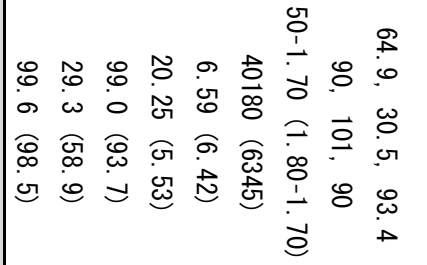 & D & $\overrightarrow{\vec{v}}$ & $\begin{array}{l}\text { Do } \\
\text { 离 } \\
\text { 离 } \\
\text { 足 } \\
\text { 言 }\end{array}$ \\
\hline 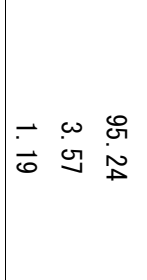 & $\begin{array}{ll}\overrightarrow{0} & 0 \\
\dot{\phi} & \stackrel{0}{\omega}\end{array}$ & 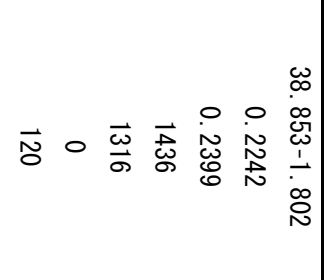 & 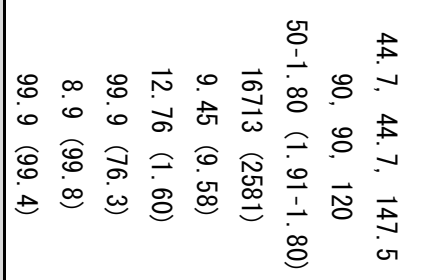 & 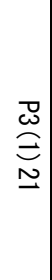 & 谤 & 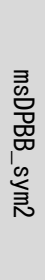 \\
\hline
\end{tabular}




\begin{tabular}{|c|c|c|c|c|c|c|}
\hline $\begin{array}{lll}0 & 8 \\
0 & 0 & 0 \\
0 & 0\end{array}$ & 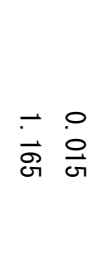 & 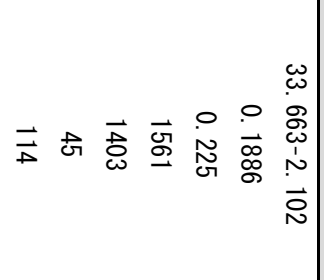 & 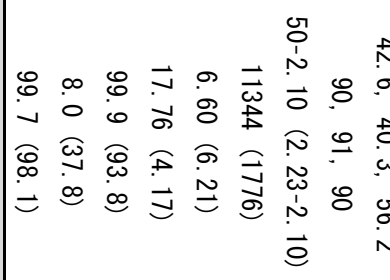 & $\stackrel{\widetilde{\Xi}}{\cong}$ & D্ & $\frac{\frac{0}{5}}{5}$ \\
\hline $00 \overrightarrow{8}$ & $\begin{array}{ll}0 & 0 \\
00 & 8 \\
00\end{array}$ & 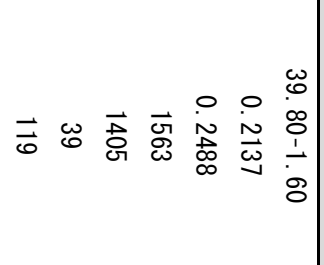 & 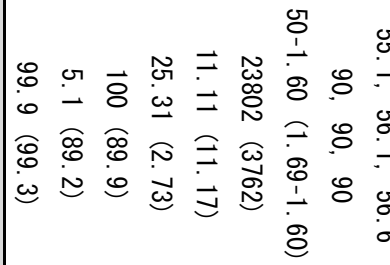 & 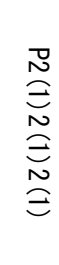 & 齐 & $\begin{array}{l}\text { 恶 } \\
\text { J }\end{array}$ \\
\hline $00 \overrightarrow{8}$ & $\begin{array}{ll}\vec{\omega} & 0 \\
\dot{\omega} & 0 \\
& 0\end{array}$ & 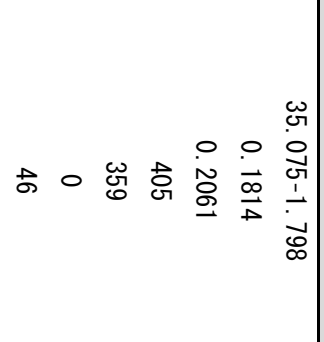 & 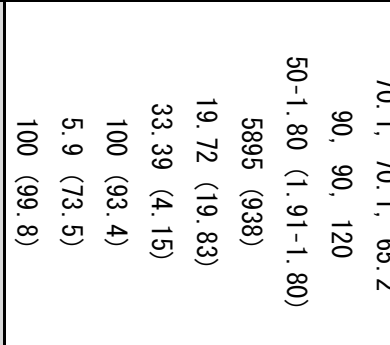 & 躴 & 콫 & 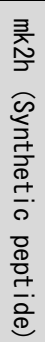 \\
\hline 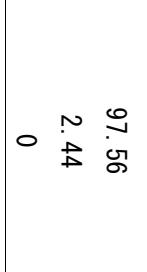 & $\begin{array}{r}0 \\
-8 \\
8\end{array}$ & 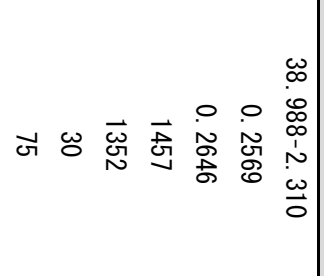 & 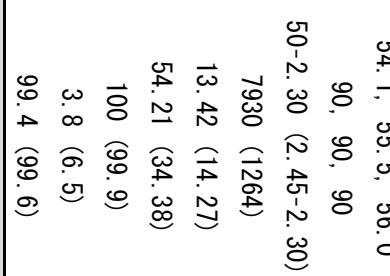 & 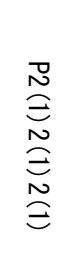 & 롳 & $\begin{array}{l}\text { 콜 } \\
\text { 灾 } \\
\text { 另 }\end{array}$ \\
\hline $00 \overrightarrow{8}$ & $\begin{array}{ll}\overrightarrow{0} & 0 \\
0 & 8\end{array}$ & 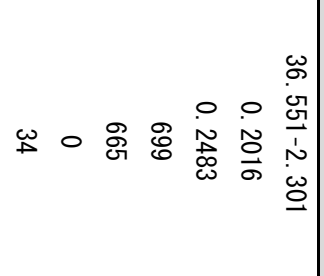 & 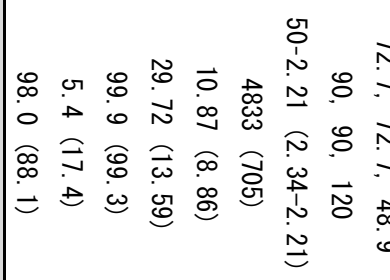 & 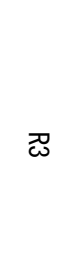 & קָ & 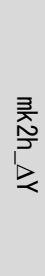 \\
\hline $00 \overrightarrow{8}$ & $\begin{array}{ll}\circ & 0 \\
\infty & 0 \\
\infty & 8\end{array}$ & 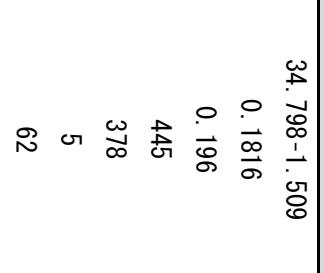 & 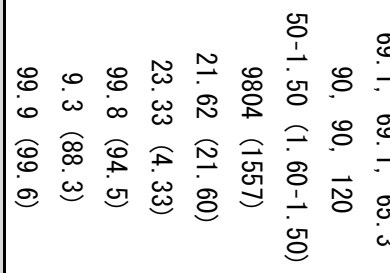 & 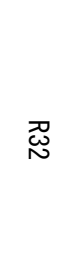 & 출 & 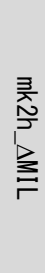 \\
\hline
\end{tabular}




\begin{tabular}{|c|c|c|c|c|c|c|c|}
\hline $00 \overrightarrow{8}$ & $\overrightarrow{0}$ & 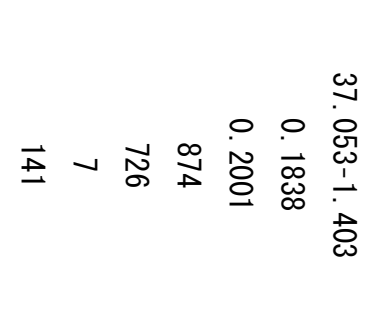 & 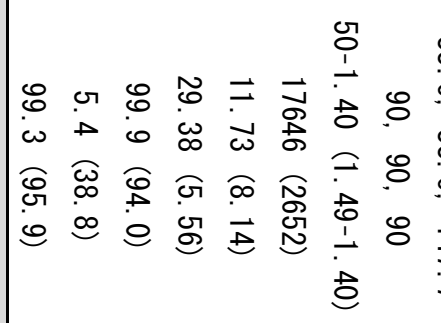 & $\begin{array}{l}\omega \\
\omega \\
0 \\
\omega \\
\omega \\
0 \\
0 \\
\vec{E} \\
. \\
\nu\end{array}$ & $\begin{array}{l}\frac{D}{\overparen{\omega}} \\
\stackrel{\omega}{N} \\
\stackrel{\Xi}{N}\end{array}$ & 뭆 & 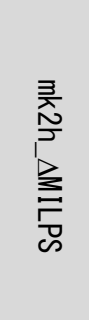 \\
\hline $00 \overrightarrow{8}$ & $\begin{array}{l}\overrightarrow{0} \\
\dot{\phi}\end{array}$ & 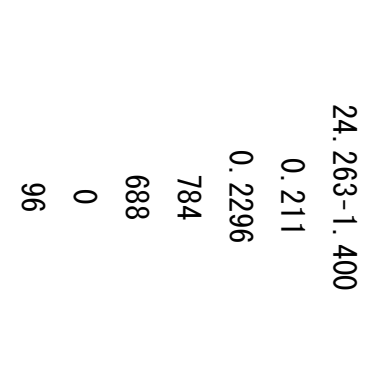 & 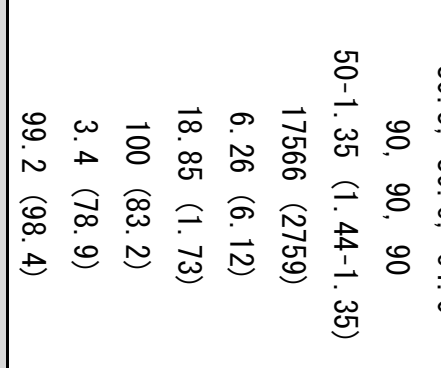 & 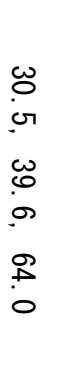 & 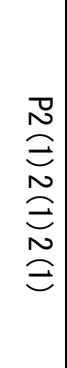 & 카 & 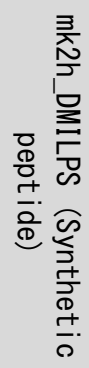 \\
\hline $00 \overrightarrow{8}$ & $\begin{array}{l}\overrightarrow{0} \\
\vec{N} \\
\end{array}$ & 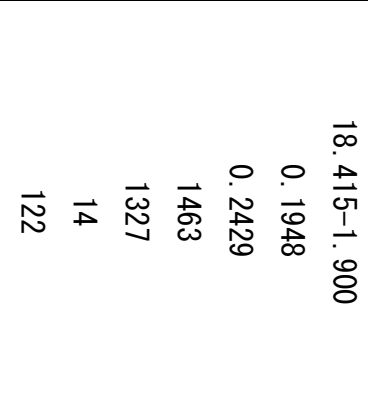 & 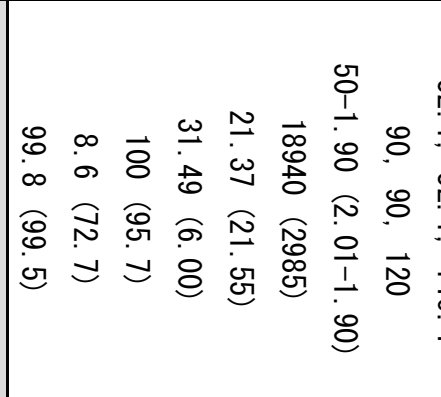 & $\begin{array}{l}\stackrel{N}{N} \\
- \\
\text { N } \\
\stackrel{-}{-} \\
\vec{D} \\
\text { D } \\
D\end{array}$ & $\begin{array}{l}\stackrel{\Xi}{心} \\
\stackrel{\Xi}{\simeq}\end{array}$ & 空 & 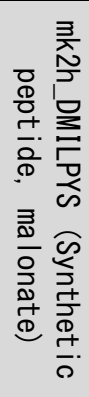 \\
\hline 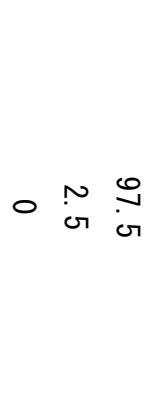 & $\begin{array}{ll}\vec{\omega} & 0 \\
\stackrel{ \pm}{*} & 0 \\
N\end{array}$ & 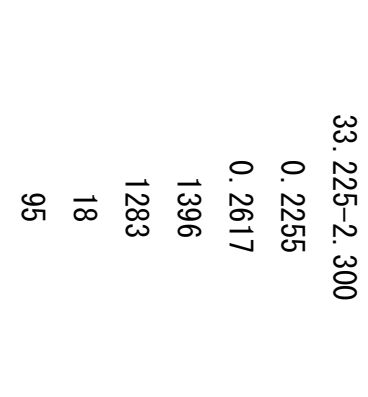 & 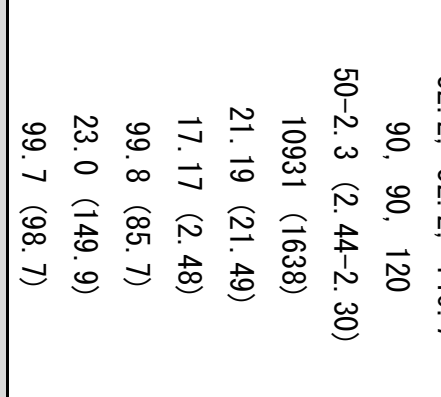 & $\begin{array}{l}\text { N } \\
\text { N } \\
\text { N } \\
\text { N } \\
\text { D } \\
\text { D }\end{array}$ & $\stackrel{\circlearrowright}{\stackrel{ٍ}{N}}$ & 흥 & 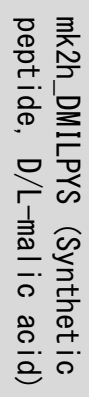 \\
\hline
\end{tabular}


bioRxiv preprint doi: https://doi.org/10.1101/2021.02.22.432383; this version posted February 23, 2021. The copyright holder for this preprint (which was not certified by peer review) is the author/funder. All rights reserved. No reuse allowed without permission.

Table S3. Ranking of VCP_DPBBs based on internal sequence identity.

\begin{tabular}{|c|c|c|}
\hline Rank & Internal sequence identity $(\%)$ & Species \\
\hline 1 & 45.455 & Aeropyrum_pernix_K1_BAA80362 \\
\hline 2 & 41.86 & Methanopyrus_kandleri_AV19_AAM01701 \\
\hline 3 & 38. 636 & Thermog ladius_cellulolyticus_1633_AFK50582 \\
\hline 4 & 37. 209 & taVCP_DPBB \\
\hline 4 & 37. 209 & Thermop lasma_acidophi I lum_DSM_1728_005209 \\
\hline 6 & 34. 884 & Thermop lasma_vol can i um_WP_010917205 \\
\hline 6 & 34. 884 & Cand idatus_Micrarchaeota_archaeon_CG1_02_47_40_0I020729.1 \\
\hline 6 & 34. 884 & Thaumarchaeota_archaeon_MY2_WP_042686022 \\
\hline 6 & 34. 884 & Pyrococcus_furiosus_WP_011012100 \\
\hline 6 & 34. 884 & Pyrococcus_horikoshi i_0T3_BAA30961 \\
\hline 6 & 34.884 & Pyrococcus_horikoshi i_WP_048053493 \\
\hline 12 & 34. 091 & Desul furococcus_mucosus_DSM_2162_ADV64559 \\
\hline 13 & 32. 558 & Staphy lothermus_mar inus_F1_ABN69820 \\
\hline 13 & 32. 558 & Thermog ladius_cellulolyticus_1633_AFK50988 \\
\hline 13 & 32. 558 & Picrophi lus_torridus_DSM_9790_AAT43041 \\
\hline 13 & 32. 558 & Candidatus_Nitrososphaera_evergladens i s_SR1_AIF84648 \\
\hline 13 & 32. 558 & Thermococcus_kodakarens is_KOD1_BAD84858 \\
\hline 13 & 32. 558 & Pyrococcus_horikoshi i_0T3_BAA29778 \\
\hline 13 & 32. 558 & Thermof $i$ l um_pendens_Hrk_5_ABL78081 \\
\hline 13 & 32. 558 & Thermococcus_kodakarensis_KOD1_BAD85346 \\
\hline 13 & 32.558 & Thermococcus_kodakarens is_KOD1_CAT68952 \\
\hline 22 & 32. 432 & Candidatus_Nitrososphaera_gargensi s_Ga9. 2_AFU57173 \\
\hline 23 & 31.818 & Candidatus_Micrarchaeota_archaeon_CG_4_10_14_0_2_um_fi Iter_60_11_PIZ91015.1 \\
\hline 23 & 31.818 & Candidatus_Nitrososphaera_evergladensi s_SR1_AIF82607 \\
\hline 25 & 30.952 & Natr ialba_magadi i_ATCC_43099_ADD03993 \\
\hline 26 & 30.233 & Archaeoglobus_veneficus_SNP6_AEA48091 \\
\hline 26 & 30.233 & Nitrososphaera_vi ennensis_EN76_AIC15426 \\
\hline 26 & 30.233 & Candidatus_Micrarchaeota_archaeon_CG10_big_fi I_rev_8_21_14_0_10_45_29_PIT84804. 1 \\
\hline 26 & 30.233 & Candidatus_Nitrososphaera_gargensi s_Ga9. 2_AFU59974 \\
\hline 26 & 30.233 & Candidatus_Nitrosopelag icus_brevis_AJA92264 \\
\hline 26 & 30.233 & Candidatus_Bathyarchaeota_archaeon_B24-2_PDM26593 \\
\hline 26 & 30.233 & Archaeoglobus_veneficus_SNP6_AEA46762 \\
\hline 26 & 30.233 & Methanococcus_maripaludis_S2_CAF29732 \\
\hline 34 & 29.545 & Nitrososphaera_vi ennensis_EN76_AIC16229 \\
\hline 35 & 28. 571 & Ha lobacter ium_sal inarum_R1_CAP15469 \\
\hline 36 & 27. 907 & Candidatus_Pacearchaeota_archaeon_CG10_big_fi I_rev_8_21_14_0_10_32_42_PJE81802. 1 \\
\hline 36 & 27. 907 & Methanonatronarchaeum_thermophi lum_0UJ19303 \\
\hline 36 & 27. 907 & Ferrog lobus_placidus_DSM_10642_ADC66306 \\
\hline 36 & 27. 907 & Ferroplasma_acidarmanus_fer1_AG060899 \\
\hline 36 & 27. 907 & Candidatus_Parvarchaeum_acidophi I lus_ARMAN-5_EFD92611 \\
\hline 36 & 27. 907 & Caldisphaera_Iagunensis_DSM_15908_AFZ71117 \\
\hline 36 & 27. 907 & Pyrolobus_fumar i i_1A_AEM37983 \\
\hline 36 & 27. 907 & Candidatus_Altiarchaeales_archaeon_ex4484_2_0YT54626 \\
\hline 36 & 27. 907 & Thaumarchaeota_archaeon_MY2_WP_042683811 \\
\hline 36 & 27. 907 & Cenarchaeum_symb iosum_A_ABK78575 \\
\hline 36 & 27. 907 & Nitrosopumi lus_mar it imus_SCM1_ABX12001 \\
\hline 36 & 27. 907 & Candidatus_Nitrosoarchaeum_I imnia_BG20_EPA04818 \\
\hline 36 & 27. 907 & Thaumarchaeota_archaeon_N4_CDI06512 \\
\hline 36 & 27. 907 & Thaumarchaeota_archaeon_N4_WP_048197167 \\
\hline 36 & 27. 907 & Candidatus_Geothermarchaeota_archaeon_ex4572_27_PCN50067 \\
\hline
\end{tabular}


bioRxiv preprint doi: https://doi.org/10.1101/2021.02.22.432383; this version posted February 23, 2021. The copyright holder for this

preprint (which was not certified by peer review) is the author/funder. All rights reserved. No reuse allowed without permission.

\begin{tabular}{|c|c|c|}
\hline 36 & 27. 907 & Candidatus_0dinarchaeota_archaeon_LCB_4_OLS17411 \\
\hline 36 & 27. 907 & Candidatus_Bathyarchaeota_archaeon_B24_KYH37740 \\
\hline 36 & 27. 907 & Pyrococcus_furiosus_WP_011013021 \\
\hline 54 & 27. 273 & Cand idatus_He imdal I archaeota_archaeon_LC_3_OLS20813 \\
\hline 54 & 27. 273 & Candidatus_He imdal I archaeota_archaeon_LC_2_OLS27801 \\
\hline 54 & 27. 273 & Igni sphaera_aggregans_DSM_17230_ADM27979 \\
\hline 54 & 27. 273 & Ignicoccus_hospital is_KIN4/I_ABU82607 \\
\hline 54 & 27. 273 & Ignicoccus_hospital is_WP_052570589 \\
\hline 54 & 27. 273 & Staphy lothermus_mar inus_F1_ABN70219 \\
\hline 54 & 27. 273 & Methanocaldococcus_jannaschi i _DSM_2661_AAB99153 \\
\hline 61 & 26.19 & Ha lorubrum_lacusprofundi_ATCC_49239_ACM57952 \\
\hline 62 & 26. 087 & Candidatus_Nanosal inarum_sp._J07AB56_EG040547 \\
\hline 62 & 26. 087 & Nanoha loarchaea_archaeon_SG9_A0V94540 \\
\hline 64 & 25.581 & halophi I ic_archaeon_DL31_WP_014052869 \\
\hline 64 & 25.581 & Candidatus_Di apherotrites_archaeon_CG08_land_8_20_14_0_20_34_12_PIU21023.1 \\
\hline 64 & 25. 581 & The i onarchaea_archaeon_DG-70_KYK34434 \\
\hline 64 & 25.581 & Candidatus_Methanohalarchaeum_thermophi l lum_0KY77569 \\
\hline 64 & 25.581 & Methanohalophi l us_mahi i_DSM_5219_ADE35783 \\
\hline 64 & 25.581 & Methanosarcina_barkeri_227_AKB57915 \\
\hline 64 & 25.581 & Candidatus_He imdal I archaeota_archaeon_LC_2_OLS28009 \\
\hline 64 & 25.581 & Candidatus_Parvarchaeum_acidiphi l lum_ARMAN-4_EEZ93171 \\
\hline 64 & 25.581 & Ignicoccus_hospital is_KIN4/I_ABU81875 \\
\hline 64 & 25.581 & Acidul iprofundum_boone i_T469_EDY34590 \\
\hline 64 & 25.581 & Nitrososphaera_viennensis_EN76_AIC15017 \\
\hline 64 & 25.581 & Candidatus_Ni trososphaera_gargensis_Ga9. 2_AFU59997 \\
\hline 64 & 25.581 & Candidatus_Nitrososphaera_evergladens is_SR1_AIF85237 \\
\hline 64 & 25. 581 & Candidatus_Nitrosotalea_devanaterra_CUR52664 \\
\hline 64 & 25.581 & Cand idatus_Bathyarchaeota_archaeon_B24-2_PDM25984 \\
\hline 79 & 25 & Pyrolobus_fumar i i_1A_AEM37966 \\
\hline 79 & 25 & Marine_Group_III_euryarchaeote_CG-Bathy1_0IR19087 \\
\hline 79 & 25 & Candidatus_Caldiarchaeum_subterraneum_BAJ47789 \\
\hline 79 & 25 & Methanothermus_fervidus_DSM_2088_ADP77767 \\
\hline 79 & 25 & Pyrolobus_fumari i_1A_AEM38259 \\
\hline 84 & 24.324 & Candidatus_Nitrosotal ea_devanaterra_CUR52322 \\
\hline 85 & 23.913 & Candidatus_Nanosal ina_sp. _J07AB43_EGQ43838 \\
\hline 86 & 23. 81 & Candidatus_Micrarchaeota_archaeon_Mi a14_WP_088820610. 1 \\
\hline 87 & 23. 256 & Thaumarchaeota_archaeon_N4_CDI06696 \\
\hline 87 & 23. 256 & Thaumarchaeota_archaeon_MY2_WP_042684250 \\
\hline 87 & 23. 256 & Candidatus_Pacearchaeota_archaeon_CG10_big_fi I_rev_8_21_14_0_10_34_76_PIN89331. 1 \\
\hline 87 & 23. 256 & Candidatus_Pacearchaeota_archaeon_CG_4_9_14_0_2_um_fi Iter_39_13_PJC44446.1 \\
\hline 87 & 23.256 & The i onarchaea_archaeon_DG-70-1_KYK31403 \\
\hline 87 & 23. 256 & Candidatus_0dinarchaeota_archaeon_LCB_4_0LS17850 \\
\hline 87 & 23. 256 & Marine_Group_II_euryarchaeote_MED-G33_PDH24769. 1 \\
\hline 87 & 23. 256 & Mar ine_Group_II_euryarchaeote_MED-G34_PDH26101. 1 \\
\hline 87 & 23. 256 & uncul tured_Candidatus_Thalassoarchaea_euryarchaeote_AK006073 \\
\hline 87 & 23. 256 & Archaeog lobus_fulgidus_DSM_4304_AAB89157 \\
\hline 87 & 23. 256 & Methanocel Ia_paludicola_SANAE_BAI 62891 \\
\hline 87 & 23. 256 & halophi I ic_archaeon_DL31_WP_014051352 \\
\hline 87 & 23. 256 & Halorubrum_lacusprofundi_ATCC_49239_ACM57299 \\
\hline 87 & 23. 256 & Cand idatus_Woesearchaeota_archaeon_CG10_big_fi I_rev_8_21_14_0_10_44_13_PII \\
\hline
\end{tabular}


bioRxiv preprint doi: https://doi.org/10.1101/2021.02.22.432383; this version posted February 23, 2021. The copyright holder for this

preprint (which was not certified by peer review) is the author/funder. All rights reserved. No reuse allowed without permission.

\begin{tabular}{|c|c|c|}
\hline 87 & 23. 256 & Aeropyrum_pernix_K1_BAA81490 \\
\hline 87 & 23. 256 & Acidilobus_saccharovorans_345-15_ADL18813 \\
\hline 87 & 23. 256 & Fervidicoccus_fontis_Kam940_AFH42351 \\
\hline 87 & 23. 256 & Candidatus_He imdal I archaeota_archaeon_AB_125_0LS33307 \\
\hline 87 & 23. 256 & Candidatus_Altiarchaeal es_archaeon_IMC4_ODS42491 \\
\hline 87 & 23. 256 & Methanohal ophi I lus_mahi i_DSM_5219_ADE36585 \\
\hline 87 & 23. 256 & Methanohal ophi I us_mahi i_WP_048902269 \\
\hline 87 & 23. 256 & Methanosarcina_barkeri_227_AKB57217 \\
\hline 87 & 23. 256 & Candidatus_Bathyarchaeota_archaeon_BA2_KPV62335 \\
\hline 87 & 23. 256 & Cand idatus_Bathyarchaeota_archaeon_B26-2_KYH41545 \\
\hline 87 & 23. 256 & Ferroglobus_placidus_WP_048086329 \\
\hline 87 & 23. 256 & Archaeog lobus_fulgidus_DSM_4304_AAB89948 \\
\hline 87 & 23. 256 & Acidul iprofundum_boonei_T469_EDY35166 \\
\hline 114 & 22. 727 & Cand idatus_Mi crarchaeum_acid i phi I lum_ARMAN-2_EET90444 \\
\hline 114 & 22. 727 & Candidatus_Micrarchaeota_archaeon_Mia14_ASI 13900. 1 \\
\hline 114 & 22. 727 & Candidatus_Nitrososphaera_evergladensis_SR1_AIF85315 \\
\hline 114 & 22. 727 & Thermosphaera_aggregans_DSM_11486_ADG91457 \\
\hline 114 & 22. 727 & Methanobacter ium_formicicum_DSM_3637_EKF86248 \\
\hline 119 & 21.951 & Caldisphaera_lagunens is_DSM_15908_AFZ70901 \\
\hline 120 & 21.739 & Candidatus_Haloredivivus_sp._G17_EHK01882 \\
\hline 121 & 21.429 & Candidatus_Methanohalarchaeum_thermophi l um_0KY78788 \\
\hline 121 & 21.429 & Ha lobacter ium_sal inarum_R1_CAP14060 \\
\hline 123 & 20.93 & Candidatus_Thorarchaeota_archaeon_AB_25_0LS24014 \\
\hline 123 & 20.93 & Halorubrum_lacusprofundi_ATCC_49239_ACM57647 \\
\hline 123 & 20.93 & Halorubrum_lacusprofundi_WP_049933528 \\
\hline 123 & 20.93 & Desulfurococcus_mucosus_DSM_2162_ADV65387 \\
\hline 123 & 20.93 & Thermosphaera_aggregans_DSM_11486_ADG91259 \\
\hline 123 & 20. 93 & Methanocel Ia_paludicola_SANAE_BAI60129 \\
\hline 123 & 20. 93 & Candidatus_Pacearchaeota_archaeon_CG10_big_fi I_rev_8_21_14_0_10_35_219_PI008257.1 \\
\hline 123 & 20.93 & uncul tured_Candidatus_Thalassoarchaea_euryarchaeote_ANV81027 \\
\hline 123 & 20. 93 & Marine_Group_II_euryarchaeote_MED-G38_PDH22657. 1 \\
\hline 123 & 20. 93 & Ha lobacter i um_sal inarum_R1_CAP14207 \\
\hline 123 & 20. 93 & Natr ialba_magadi i_ATCC_43099_ADD03768 \\
\hline 123 & 20.93 & Cand idatus_Methanoperedens_nitroreducens_KCZ71217 \\
\hline 123 & 20.93 & Candidatus_Nitrosope lag icus_brevis_AJA91966 \\
\hline 123 & 20.93 & Caldisphaera_lagunens is_WP_048816895 \\
\hline 123 & 20.93 & Nanoarchaeum_equitans_Kin4-M_AAR39317 \\
\hline 123 & 20. 93 & Thermof $\mathrm{i}$ lum_pendens_Hrk_5_ABL77714 \\
\hline 123 & 20. 93 & Thermof i lum_pendens_WP_052885007 \\
\hline 123 & 20. 93 & Candidatus_Methanoperedens_nitroreducens_KCZ71917 \\
\hline 123 & 20.93 & Pyrodictium_delaneyi_ALL01915 \\
\hline 123 & 20. 93 & Candidatus_Thorarchaeota_archaeon_SMTZ1-83_KXH77531 \\
\hline 123 & 20. 93 & Candidatus_Thorarchaeota_archaeon_SMTZ1-45_KXH73371 \\
\hline 123 & 20. 93 & Candidatus_Thorarchaeota_archaeon_AB_25_0LS31443 \\
\hline 123 & 20.93 & Methanobacter ium_formicicum_DSM_3637_EKF85426 \\
\hline 123 & 20.93 & Candidatus_Bathyarchaeota_archaeon_CG07_land_8_20_14_0_80_47_9_PIU59706 \\
\hline 123 & 20.93 & Pyrobaculum_aerophi lum_str._IM2_AAL64724 \\
\hline 123 & 20. 93 & Thermoproteus_tenax_Kra_1_CCC81686 \\
\hline 123 & 20.93 & Thermoproteus_tenax_WP_052883121 \\
\hline 150 & 20.455 & Candidatus_Nitrososphaera_gargens is_Ga9. 2_AFU59866 \\
\hline
\end{tabular}


bioRxiv preprint doi: https://doi.org/10.1101/2021.02.22.432383; this version posted February 23, 2021. The copyright holder for this preprint (which was not certified by peer review) is the author/funder. All rights reserved. No reuse allowed without permission.

\begin{tabular}{|c|c|c|}
\hline 150 & 20.455 & Cand idatus_Di apherotrites_archaeon_CG08_I and_8_20_14_0_20_30_16_PIU22450.1 \\
\hline 150 & 20.455 & Natrialba_magadi i_ATCC_43099_ADD06942 \\
\hline 150 & 20.455 & Candidatus_Huberarchaea_archaeon_CG03_Iand_8_20_14_0_80_31_114_PIV13743. 1 \\
\hline 150 & 20. 455 & Candidatus_Bathyarchaeota_archaeon_BA2_KPV63518 \\
\hline 150 & 20.455 & Candidatus_Methanoperedens_nitroreducens_KCZ72076 \\
\hline 150 & 20.455 & Fervidicoccus_font is_Kam940_AFH43154 \\
\hline 150 & 20.455 & Caldivirga_maquilingensis_IC-167_ABW01805 \\
\hline 158 & 20 & Metal losphaera_yel lowstonens is_MK1_EHP69309 \\
\hline 158 & 20 & Hyperthermus_butyl icus_DSM_5456_ABM80172 \\
\hline 158 & 20 & Hyperthermus_butyl icus_WP_048061379 \\
\hline 161 & 19. 565 & Acidi lobus_saccharovorans_345-15_ADL19616 \\
\hline 162 & 19. 048 & Candidatus_Diapherotrites_archaeon_CG10_big_fi I_rev_8_21_14_0_10_31_34_PIN99301.1 \\
\hline 163 & 18. 605 & Candidatus_Diapherotrites_archaeon_CG09_I and_8_20_14_0_10_32_12_PIU02624. 1 \\
\hline 163 & 18. 605 & Cenarchaeum_symb iosum_A_ABK78458 \\
\hline 163 & 18. 605 & Candidatus_Thorarchaeota_archaeon_SMTZ1-83_KXH72124 \\
\hline 163 & 18. 605 & Candidatus_Thorarchaeota_archaeon_SMTZ1-45_KXH74223 \\
\hline 163 & 18. 605 & Candidatus_Nitrososphaera_evergladens is_SR1_AIF83909 \\
\hline 163 & 18. 605 & Candidatus_Pacearchaeota_archaeon_CG10_big_fi I_rev_8_21_14_0_10_32_42_PJE81799.1 \\
\hline 163 & 18. 605 & Candidatus_Geothermarchaeota_archaeon_ex4572_27_PCN50199 \\
\hline 163 & 18. 605 & Candidatus_Caldiarchaeum_subterraneum_BAJ47117 \\
\hline 163 & 18. 605 & The ionarchaea_archaeon_DG-70_KYK31941 \\
\hline 163 & 18. 605 & Lokiarchaeum_sp._GC14_75_KKK40880 \\
\hline 163 & 18. 605 & Candidatus_Woesearchaeota_archaeon_CG10_big_fi I_rev_8_21_14_0_10_36_11_PIN75767.1 \\
\hline 163 & 18. 605 & Candidatus_Nitrososphaera_gargensis_Ga9.2_AFU57477 \\
\hline 163 & 18. 605 & Nanoarchaeota_archaeon_7A_AMD29691 \\
\hline 163 & 18. 605 & nanoarchaeote_Nst1_E0D42766 \\
\hline 163 & 18. 605 & Candidatus_Methanoperedens_nitroreducens_KCZ73677 \\
\hline 178 & 18. 182 & Candidatus_Nitrosotalea_devanaterra_CUR51378 \\
\hline 178 & 18. 182 & Cand idatus_Methanomethy lophi lus_al vus_Mx1201_AGI86363 \\
\hline 178 & 18. 182 & Cand idatus_Korarchaeum_cryptof i lum_OPF8_ACB06954 \\
\hline 178 & 18. 182 & Caldivirga_maquilingensis_IC-167_ABW01385 \\
\hline 178 & 18. 182 & Cand idatus_Aen igmarchaeota_archaeon_ex4484_224_0YT42612. 1 \\
\hline 178 & 18. 182 & Candidatus_Aen igmarchaeota_archaeon_CG_4_10_14_3_um_fi Iter_37_21_PIY34793.1 \\
\hline 178 & 18. 182 & Candidatus_He imdal I archaeota_archaeon_LC_2_OLS28431 \\
\hline 178 & 18. 182 & Candidatus_Bathyarchaeota_archaeon_B24_KYH36593 \\
\hline 178 & 18. 182 & Methanobrevibacter_smithi i_ATCC_35061_ABQ86847 \\
\hline 178 & 18. 182 & Methanosarcina_barkeri_227_AKB58810 \\
\hline 178 & 18. 182 & Methanocel Ia_paludicola_SANAE_BAI62887 \\
\hline 178 & 18. 182 & Metal I osphaera_yel l owstonens is_MK1_EHP69577 \\
\hline 178 & 18. 182 & Vulcanisaeta_moutnovskia_768-28_ADY00631 \\
\hline 191 & 17. 778 & Pyrodictium_delaneyi_ALL01313 \\
\hline 192 & 16. 279 & Candidatus_Nitrosopumi lus_salaria_WP_008298811. 1 \\
\hline 192 & 16. 279 & Candidatus_Nitrosopumi lus_salar ia_WP_008297191.1 \\
\hline 192 & 16. 279 & Nitrosopumi lus_maritimus_SCM1_ABX11911 \\
\hline 192 & 16. 279 & Candidatus_Nitrosotalea_devanaterra_CUR52859 \\
\hline 192 & 16. 279 & Candidatus_Nitrosotenuis_cloacae_AJZ75973 \\
\hline 192 & 16. 279 & Lokiarchaeum_sp._GC14_75_KKK44519 \\
\hline 192 & 16. 279 & Candidatus_Pacearchaeota_archaeon_CG10_big_fi I_rev_8_21_14_0_10_35_219_PI007960.1 \\
\hline 192 & 16. 279 & Marine_Group_II_euryarchaeote_MED-G37_PDH23088. 1 \\
\hline 192 & 16. 279 & Candidatus_Bathyarchaeota_archaeon_B24-2_PDM26092 \\
\hline
\end{tabular}


bioRxiv preprint doi: https://doi.org/10.1101/2021.02.22.432383; this version posted February 23, 2021. The copyright holder for this preprint (which was not certified by peer review) is the author/funder. All rights reserved. No reuse allowed without permission.

\begin{tabular}{|c|c|c|}
\hline 192 & 16. 279 & Candidatus_Bathyarchaeota_archaeon_B26-2_KYH42037 \\
\hline 192 & 16. 279 & archaeon_GW2011_AR15_AJF61321 \\
\hline 192 & 16. 279 & Hyperthermus_butyl icus_DSM_5456_ABM81185 \\
\hline 192 & 16. 279 & Hyperthermus_butyl icus_WP_048061869 \\
\hline 192 & 16. 279 & Cand idatus_Lokiarchaeota_archaeon_CR_4_OLS12095 \\
\hline 192 & 16. 279 & Cand idatus_Methanohal archaeum_thermophi I um_0KY78140 \\
\hline 192 & 16. 279 & Methanonatronarchaeum_thermophi lum_0UJ19199 \\
\hline 192 & 16. 279 & Methanosarcina_barkeri_227_AKB58911 \\
\hline 192 & 16. 279 & Candidatus_0dinarchaeota_archaeon_LCB_4_0LS17239 \\
\hline 210 & 15. 909 & archaeon_GW2011_AR5_KH047409 \\
\hline 210 & 15.909 & Cand idatus_Korarchaeum_cryptofi l um_OPF8_ACB06989 \\
\hline 212 & 14. 286 & Natrialba_magadi i_ATCC_43099_ELY26084 \\
\hline 212 & 14. 286 & halophilic_archaeon_DL31_WP_014051559 \\
\hline 214 & 13. 953 & Candidatus_He imdal I archaeota_archaeon_LC_2_OLS24790 \\
\hline 214 & 13. 953 & Candidatus_Diapherotrites_archaeon_CG09_Iand_8_20_14_0_10_32_12_PIU02962. 1 \\
\hline 214 & 13. 953 & Candidatus_Nitrosotalea_devanaterra_CUR51973 \\
\hline 214 & 13. 953 & Cenarchaeum_symbiosum_A_ABK77156 \\
\hline 218 & 13. 636 & Natr ialba_magad i i_ATCC_43099_ADD04359 \\
\hline 218 & 13. 636 & Candidatus_Heimdal I archaeota_archaeon_LC_3_OLS27535 \\
\hline 218 & 13. 636 & Candidatus_He imdal I archaeota_archaeon_LC_3_OLS19891 \\
\hline 218 & 13. 636 & Pyrobaculum_aerophi lum_str._IM2_AAL62959 \\
\hline 218 & 13.636 & Vulcani saeta_moutnovskia_768-28_ADY01148 \\
\hline 223 & 13. 514 & Candidatus_Woesearchaeota_archaeon_CG10_big_fi I_rev_8_21_14_0_10_45_16_PIN74326.1 \\
\hline 224 & 13. 333 & Candidatus_Aenigmarchaeota_archaeon_ex4484_56_0YT43213.1 \\
\hline 225 & 12. 766 & Sulfolobus_tokodai i_str._7_BAK54193 \\
\hline 225 & 12. 766 & Sulfolobus_tokodai i_WP_052846861 \\
\hline 227 & 11.905 & Candidatus_Woesearchaeota_archaeon_CG10_big_fi I_rev_8_21_14_0_10_44_13_PIN86711.1 \\
\hline 228 & 11.628 & Candidatus_Nitrosoarchaeum_koreens is_MY1_EGP94659 \\
\hline 228 & 11.628 & Candidatus_Nitrosoarchaeum_I imnia_WP_010191855 \\
\hline 228 & 11.628 & Cand idatus_Alt i archaeum_sp._CG2_30_32_3053_0IQ05114 \\
\hline 228 & 11.628 & Thaumarchaeota_archaeon_N4_CDI05953 \\
\hline 228 & 11.628 & Candidatus_Nitrosotenuis_cloacae_AJZ76520 \\
\hline 228 & 11.628 & Nitrosopumi lus_maritimus_SCM1_ABX13119 \\
\hline 228 & 11.628 & Candidatus_Nitrosoarchaeum_I imnia_WP_010191195 \\
\hline 228 & 11.628 & Candidatus_Nitrososphaera_evergladens is_SR1_AIF83719 \\
\hline 228 & 11.628 & Candidatus_Nitrososphaera_gargens is_Ga9.2_AFU58810 \\
\hline 228 & 11.628 & archaeon_GW2011_AR20_AJF62478 \\
\hline 238 & 11.364 & Candidatus_Geothermarchaeota_archaeon_ex4572_27_PCN51246 \\
\hline 238 & 11.364 & Sulfolobus_tokodai i_str._7_BAK54256 \\
\hline 240 & 9.524 & Cand idatus_Alt i archaeal es_archaeon_WOR_SM1_SCG_ODS35378 \\
\hline 241 & 9. 302 & Thaumarchaeota_archaeon_MY2_WP_042687141 \\
\hline 241 & 9. 302 & Candidatus_Nitrosoarchaeum_koreens is_WP_048109923 \\
\hline 241 & 9. 302 & Nitrososphaera_viennensis_EN76_AIC14530 \\
\hline 244 & 9. 091 & Lokiarchaeum_sp._GC14_75_KKK42851 \\
\hline 244 & 9. 091 & Thermoproteus_tenax_Kra_1_CCC81122 \\
\hline 244 & 9. 091 & Hadesarchaea_archaeon_YNP_N21_KU042916 \\
\hline 247 & 7.5 & Candidatus_Nitrososphaera_evergl adens is_SR1_AIF82434 \\
\hline 248 & 4. 651 & Candidatus_Nitrosopumi I us_sal ar ia_WP_008297438. 1 \\
\hline 249 & 4. 545 & Nitrososphaera_viennensis_EN76_AIC16426 \\
\hline
\end{tabular}




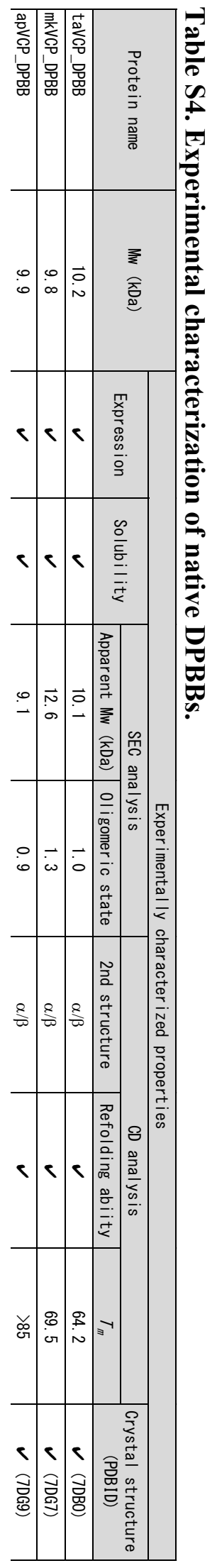




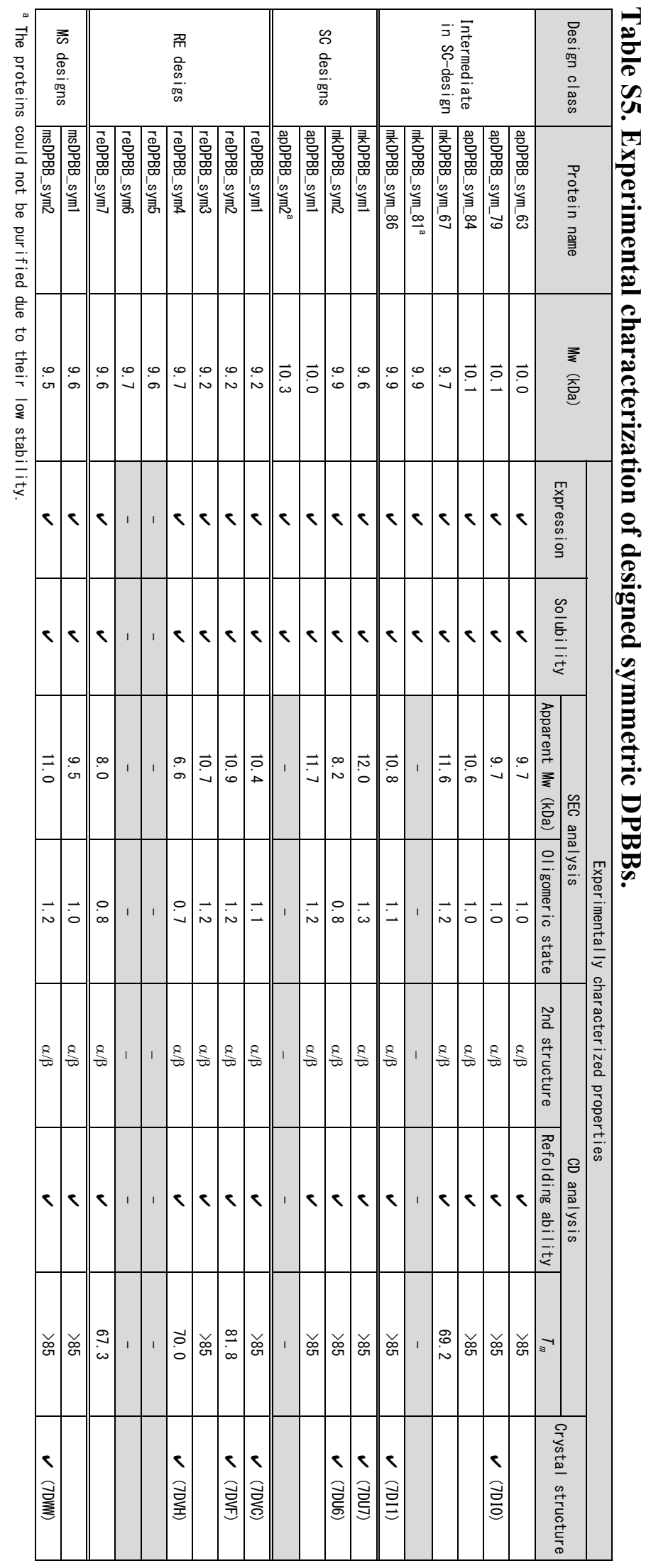




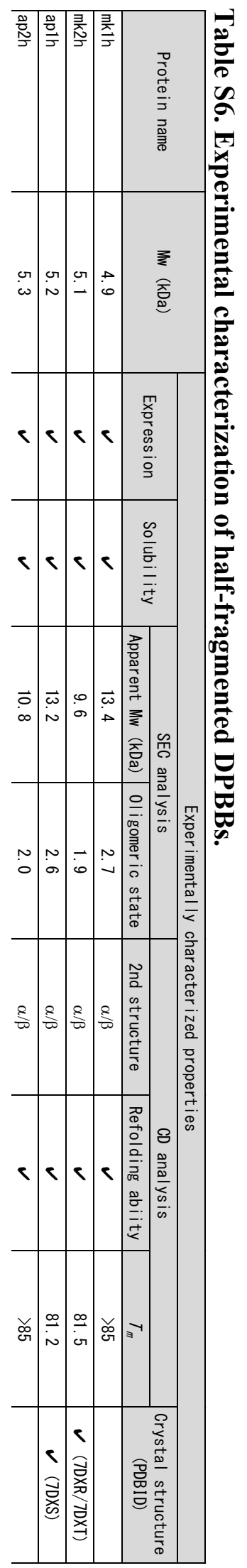




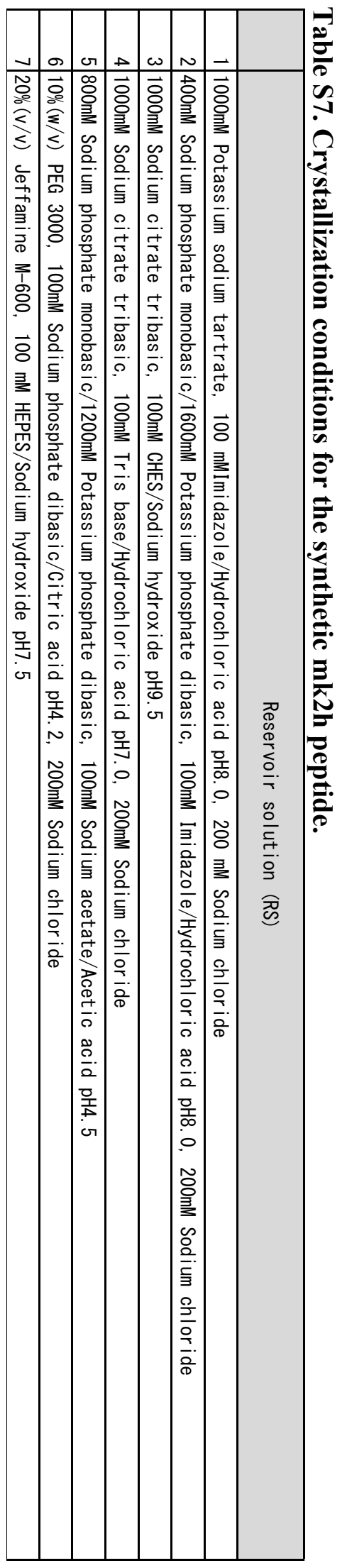


bioRxiv preprint doi: https://doi.org/10.1101/2021.02.22.432383; this version posted February 23,2021 . The copyright holder for this

preprint (which was not certified by peer review) is the author/funder. All rights reserved. No reuse allowed without permission.

Table S8. Quantification of peptide species by LC/MS.

\begin{tabular}{|c|c|c|c|c|c|c|c|}
\hline \multicolumn{8}{|l|}{ mk1h peptide } \\
\hline \multirow[b]{2}{*}{ Monoisotopic mass (Da) } & \multicolumn{2}{|c|}{ Before SEC } & \multicolumn{2}{|c|}{ Aggregation frac. } & \multicolumn{3}{|c|}{ Dimer frac. } \\
\hline & Sum Intensity & Relative Abundance $(\%)$ & Sum Intensity & Relative Abundance $(\%)$ & Sum Intensity & Relative & Abundance $(\%)$ \\
\hline 2914.6 & 223335 & 16.7 & 1538848 & 19.3 & 1666535 & & 8.8 \\
\hline 3428.9 & 285446 & 2.1 & 50208 & 0.6 & 362748 & & 1.9 \\
\hline 4004. 1 & 359518 & 2. 7 & 673725 & 8.5 & 11170 & & 0.1 \\
\hline 4174.3 & 110288 & 0.8 & 388998 & 4. 9 & ND & $D^{\mathrm{a}}$ & \\
\hline 4316.4 & 41922 & 3.1 & 1242401 & 15.6 & 23622 & & 0.1 \\
\hline 4403.4 & 181392 & 1. 4 & 519933 & 6.5 & ND & $D^{a}$ & \\
\hline 4408.5 & 51985 & 0.4 & 357992 & 4.5 & ND & $\mathrm{ID}^{\mathrm{a}}$ & \\
\hline 4415.5 & 2939688 & 22. 0 & 7965074 & 100.0 & 271045 & & 1.4 \\
\hline 4431.5 & 287595 & 2. 2 & 697384 & 8.8 & 46841 & & 0.2 \\
\hline 4443.4 & 97978 & 0.7 & 358958 & 4. 5 & 10669 & & 0.1 \\
\hline 4514.5 & 26677 & 2. 0 & 950075 & 11.9 & 21476 & & 0.1 \\
\hline 4559.5 & 1436130 & 10.7 & 3926160 & 49. 3 & 218946 & & 1.2 \\
\hline 4571.5 & $18402 \mathrm{~S}$ & 1.4 & 471198 & 5.9 & 97616 & & 0.5 \\
\hline 4601.6 & 323717 & 2. 4 & 455629 & 5.7 & 19369 & & 0.1 \\
\hline 4658. 5 (mk1h FL) & 13366797 & 100.0 & 1608175 & 20.2 & 18906107 & & 100.0 \\
\hline 4674.5 (mk1h FL met_0X) & 558788 & 4. 2 & 262902 & 3.3 & 719772 & & 3. 8 \\
\hline \multicolumn{8}{|l|}{ mk2h peptide } \\
\hline \multirow[b]{2}{*}{ Monoisotopic mass $(\mathrm{Da})$} & \multicolumn{2}{|c|}{ Before SEC } & \multicolumn{2}{|c|}{ Aggregation frac. } & \multicolumn{3}{|c|}{ Dimer frac. } \\
\hline & Sum Intensity & Relative Abundance $(\%)$ & Sum Intensity & Relative Abundance $(\%)$ & Sum Intensity & Relative & Abundance $(\%)$ \\
\hline 1010.6 & 299575 & 2.7 & 370555 & 2. 6 & 198979 & & 1.2 \\
\hline 3575.0 & 382652 & 3.5 & 1076537 & 7. 5 & 13218 & & 0.1 \\
\hline 3600.9 & 601648 & 5.5 & 28685 & 0.2 & 70043 & & 0.4 \\
\hline 3662.0 & 621514 & 5. 6 & 72742 & 0.5 & 814803 & & 5. 0 \\
\hline 4321.4 & 21642 & 2. 0 & 337394 & 2. 4 & 4246 & & 0.0 \\
\hline 4477.5 & 277006 & 2.5 & 600300 & 4. 2 & ND & $\mathrm{ID}^{\mathrm{a}}$ & \\
\hline 4592.5 & 31193 & 2.8 & 993232 & 6.9 & ND & $D^{\mathrm{a}}$ & \\
\hline 4620.5 & 150073 & 1.4 & 387705 & 2. 7 & 35378 & & 0.2 \\
\hline 4633.6 & 225325 & 2.0 & 604106 & 4. 2 & ND & $\mathrm{ID}^{\mathrm{a}}$ & \\
\hline 4658.6 & 306598 & 2. 8 & 17624 & 0.1 & 10857 & & 0.1 \\
\hline 4720.6 & 210070 & 1.9 & 589808 & 4. 1 & 10318 & & 0.1 \\
\hline 4748.6 & 6001828 & 54.5 & 14336914 & 100.0 & 436117 & & 2.7 \\
\hline 4764. 6 & 442905 & 4. 0 & 688135 & 4. 8 & 104955 & & 0.6 \\
\hline 4819. 6 & 24108 & 2. 2 & 301508 & 2. 1 & 180023 & & 1.1 \\
\hline 4835.6 (mk2h FL) & 11002630 & 100.0 & 850392 & 5.9 & 16360720 & & 100.0 \\
\hline 4851. 6 (mk2h FL met_0X) & 524838 & 4. 8 & 150537 & 1. 0 & 1079166 & & 6. 6 \\
\hline 4862.6 & 130898 & 1.2 & 285408 & 2.0 & ND & $D^{a}$ & \\
\hline
\end{tabular}


bioRxiv preprint doi: https://doi.org/10.1101/2021.02.22.432383; this version posted February 23, 2021. The copyright holder for this

preprint (which was not certified by peer review) is the author/funder. All rights reserved. No reuse allowed without permission.

Table S9. Amino acid usage in the designed DPBBs.

\begin{tabular}{|c|c|c|c|c|c|c|c|c|c|c|c|c|c|c|c|c|c|c|c|c|c|c|}
\hline & & \multicolumn{20}{|c|}{ Amino acids } & \multirow{2}{*}{$\begin{array}{c}\text { Total a. a. } \\
\text { types }\end{array}$} \\
\hline & & A & c & D & $\mathrm{E}$ & $\mathrm{F}$ & 6 & н & 1 & $\mathrm{k}$ & L & " & n & $\mathrm{p}$ & a & $R$ & s & t & v & w & y & \\
\hline \multirow[t]{3}{*}{ Native DPBBs } & taVCP_DPBB & 5 & 1 & 7 & $7(8)$ & 0 & 7 & 0 & 7 & 6 & 4 & 2 (3) & $4(6)$ & 2 & 0 & 13 & $6(7)$ & 2 & 12 & 0 & 1 & 16 \\
\hline & mkVCP_DPBB & 8 & 0 & 7 & 8 & 0 & 8 (9) & 0 & 4 & 10 & 4 & 2 (3) & 1 & $4(5)$ & 1 & 9 & 4 & 3 & 12 & 0 & 2 & 16 \\
\hline & apYCP_DPBB & $7(8)$ & 0 & 8 & 6 & 1 & 9 & 0 & 4 & 4 & 5 & 1(2) & 0 (1) & 3 & 1 & 11 & 5 & 4 & 16 & 1 & 2 & 18 \\
\hline \multirow[t]{19}{*}{ Symmetr ic designed DPBBS } & mkPPBB_sym_65 & 7 & 0 & 7 & 5 (6) & 0 & 7 (8) & 0 & 2 & 11 & 6 & 2 (3) & 1 & $4(5)$ & 0 & 8 & 6 & 1 & 17 & 0 & 2 & 15 \\
\hline & mkPPBB_sym_79 & 6 & 0 & 6 & 5 (6) & 0 & 7 (8) & 0 & 2 & 11 & 6 & 2 (3) & 1 & $3(4)$ & 0 & 10 & 5 & 0 & 19 & 0 & 3 & 14 \\
\hline & mkPPBB_sym_84 & 8 & 0 & 6 & 7 (8) & 0 & 7 (8) & 0 & 2 & 12 & 4 & 2 (3) & 0 & $3(4)$ & 0 & 7 & 5 & 0 & 19 & 0 & 5 & 13 \\
\hline & apDPBB_sym_63 & $7(8)$ & 0 & 7 & 6 & 1 & 8 & 0 & 2 & 6 & 4 & 2 (3) & 0 (1) & 3 & 1 & 11 & 7 & 3 & 17 & 1 & 2 & 17 \\
\hline & apDPBB_sym_79 & 6 (7) & 0 & 7 & 7 & 0 & 7 & 0 & 2 & 7 & 6 & 2 (3) & 0 (1) & 3 & 1 & 12 & 5 & 1 & 19 & 0 & 3 & 15 \\
\hline & aDPPBB_sym_84 & $7(8)$ & 0 & 7 & 8 & 0 & 7 & 0 & 2 & 9 & 4 & 2 (3) & 0 (1) & 2 & 1 & 9 & 5 & 1 & 19 & 0 & 5 & 15 \\
\hline & mkDPBB_symm & 8 & 0 & 6 & 6 & 0 & 8 (9) & 0 & 2 & 12 & 6 & 2 (3) & 0 & 2 (3) & 0 & 6 & 6 & 0 & 18 & 0 & 4 & 13 \\
\hline & mkDPBB_sym2 & 8 & 0 & 6 & 8 & 0 & 6 (7) & 0 & 2 & 12 & 2 & 2 (3) & 0 & 2 (3) & 0 & 8 & 4 & 0 & 20 & 0 & 6 & 13 \\
\hline & apDPBB_sym1 & 6 (7) & 0 & 8 & 8 & 0 & 8 & 0 & 2 & 8 & 4 & 2 (3) & 0 (1) & 2 & 2 & 8 & 6 & 2 & 18 & 0 & 4 & 15 \\
\hline & apDPBB_sym2 & $8(9)$ & 0 & 6 & 8 & 0 & 6 & 0 & 2 & 10 & 4 & 2 (3) & 0 (1) & 2 & 0 & 10 & 4 & 0 & 20 & 0 & 6 & 13 \\
\hline & rvDPBB_sym1 & 6 & 0 & 8 & 6 & 0 & 10 & 0 & 6 & 10 (11) & 6 & 4 & 0 & 4 & 0 & 6 & $4(5)$ & 0 (1) & 10 & 0 & 2 & 13 \\
\hline & rVDPBB_sym2 & 6 & 0 & 6 & 8 & 0 & 10 & 0 & 4 & 10 (11) & 6 & 6 & 0 & 4 & 0 & 6 & $4(5)$ & 0 (1) & 10 & 0 & 2 & 13 \\
\hline & rVDPBB_sym3 & 6 & 0 & 8 & 6 & 0 & 10 & 0 & 8 & 10 (11) & 4 & 4 & 0 & 2 & 0 & 6 & $4(5)$ & 2 (3) & 10 & 0 & 2 & 14 \\
\hline & rVDPBB_sym4 & 8 & 0 & 6 & 6 & 0 & 8 (9) & 2 & 2 & 10 & 2 & 2 (3) & 2 & $4(5)$ & 2 & 6 & 4 & 0 & 18 & 0 & 4 & 16 \\
\hline & rVDPBB_sym5 & 10 & 0 & 8 & 6 & 0 & 8 (9) & 0 & 2 & 8 & 2 & 2 (3) & 2 & $4(5)$ & 0 & 6 & 4 & 0 & 18 & 0 & 6 & 14 \\
\hline & rVDPBB_sym6 & 8 & 0 & 6 & 8 & 0 & 8 (9) & 0 & 2 & 10 & 4 & 2 (3) & 0 & $4(5)$ & 0 & 6 & 2 & 2 & 18 & 0 & 6 & 14 \\
\hline & rVDPBB_sym7 & 10 & 0 & 6 & 8 & 0 & 8 (9) & 0 & 2 & 10 & 2 & 2 (3) & 0 & $4(5)$ & 0 & 6 & 4 & 0 & 18 & 0 & 6 & 13 \\
\hline & msDPBB_sym1 & 6 & 0 & 10 & 6 & 0 & 6 & 2 & 4 & 10 & 6 & 6 & 2 & 0 & 0 & 6 & 4 & 0 & 16 & 0 & 2 & 14 \\
\hline & msDPBB_sym2 & 6 & 0 & 8 & 8 & 0 & 6 & 2 & 6 & 6 & 10 & 6 & 2 & 0 & 0 & 6 & 4 & 0 & 16 & 0 & 0 & 13 \\
\hline \multirow[t]{4}{*}{ Half-fragmented DPBBS } & mkih & 4 & 0 & 3 & 3 & 0 & 4 (5) & 0 & 1 & 6 & 3 & 1 (2) & 0 & 1 (2) & 0 & 3 & 3 & 0 & 9 & 0 & 2 & 13 \\
\hline & mk2h & 4 & 0 & 3 & 4 & 0 & 3 (4) & 0 & 1 & 6 & 1 & 1 (2) & 0 & 1 (2) & 0 & 4 & 2 & 0 & 10 & 0 & 3 & 13 \\
\hline & apth & 3 (4) & 0 & 4 & 4 & 0 & 5 & 0 & 1 & 4 & 2 & 1 (2) & 0 (1) & 1 & 1 & 4 & 3 & 1 & 9 & 0 & 2 & 15 \\
\hline & ap2h & $4(5)$ & 0 & 3 & 4 & 0 & 3 & 0 & 1 & 5 & 2 & 1 (2) & 0 (1) & 1 & 0 & 5 & 2 & 0 & 10 & 0 & 3 & 13 \\
\hline \multirow[t]{11}{*}{ Simplified mk2h } & mk2h_sM & 4 & 0 & 3 & 4 & 0 & 3 (4) & 0 & 1 & 6 & 1 & 0 (1) & 0 & 1 (2) & 0 & 4 & 2 & 0 & 11 & 0 & 3 & 12 \\
\hline & 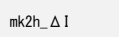 & 4 & 0 & 3 & 4 & 0 & 3 (4) & 0 & 0 & 6 & 1 & 1 (2) & 0 & 1 (2) & 0 & 4 & 2 & 0 & 11 & 0 & 3 & 12 \\
\hline & 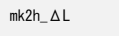 & 4 & 0 & 3 & 4 & 0 & 3 (4) & 0 & 1 & 6 & 0 & 1 (2) & 0 & 1 (2) & 0 & 4 & 2 & 0 & 11 & 0 & 3 & 12 \\
\hline & mk2h_ $\Delta P$ & 5 & 0 & 3 & 4 & 0 & 3 (4) & 0 & 1 & 6 & 1 & 1 (2) & 0 & 0 (1) & 0 & 4 & 2 & 0 & 10 & 0 & 3 & 12 \\
\hline & mk2h_ $\Delta S$ & 4 & 0 & 3 & 4 & 0 & 3 (4) & 0 & 1 & 8 & 1 & 1 (2) & 0 & 1 (2) & 0 & 4 & 0 & 0 & 10 & 0 & 3 & 12 \\
\hline & mk2h_ $\Delta Y$ & 5 & 0 & 3 & 4 & 0 & 3 (4) & 0 & 1 & 6 & 1 & 1 (2) & 0 & 1 (2) & 0 & 5 & 2 & 0 & 11 & 0 & 0 & 12 \\
\hline & mk2h_\$MIL & 4 & 0 & 3 & 4 & 0 & 3 (4) & 0 & 0 & 6 & 0 & 0 (1) & 0 & 1 (2) & 0 & 4 & 2 & 0 & 13 & 0 & 3 & 10 \\
\hline & mk2h__MILPS & 5 & 0 & 3 & 4 & 0 & 3 (4) & 0 & 0 & 8 & 0 & 0 (1) & 0 & 0 (1) & 0 & 4 & 0 & 0 & 13 & 0 & 3 & 8 \\
\hline & mk2h_\MILPY & 6 & 0 & 3 & 4 & 0 & 3 (4) & 0 & 0 & 6 & 0 & 0 (1) & 0 & 0 (1) & 0 & 5 & 2 & 0 & 14 & 0 & 0 & 8 \\
\hline & 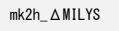 & 5 & 0 & 3 & 4 & 0 & 3 (4) & 0 & 0 & 8 & 0 & 0 (1) & 0 & 1 (2) & 0 & 5 & 0 & 0 & 14 & 0 & 0 & 8 \\
\hline & mk2h__MILPYS & 6 & 0 & 3 & 4 & 0 & 3 (4) & 0 & 0 & 8 & 0 & 0 (1) & 0 & 0 (1) & 0 & 5 & 0 & 0 & 14 & 0 & 0 & 7 \\
\hline
\end{tabular}




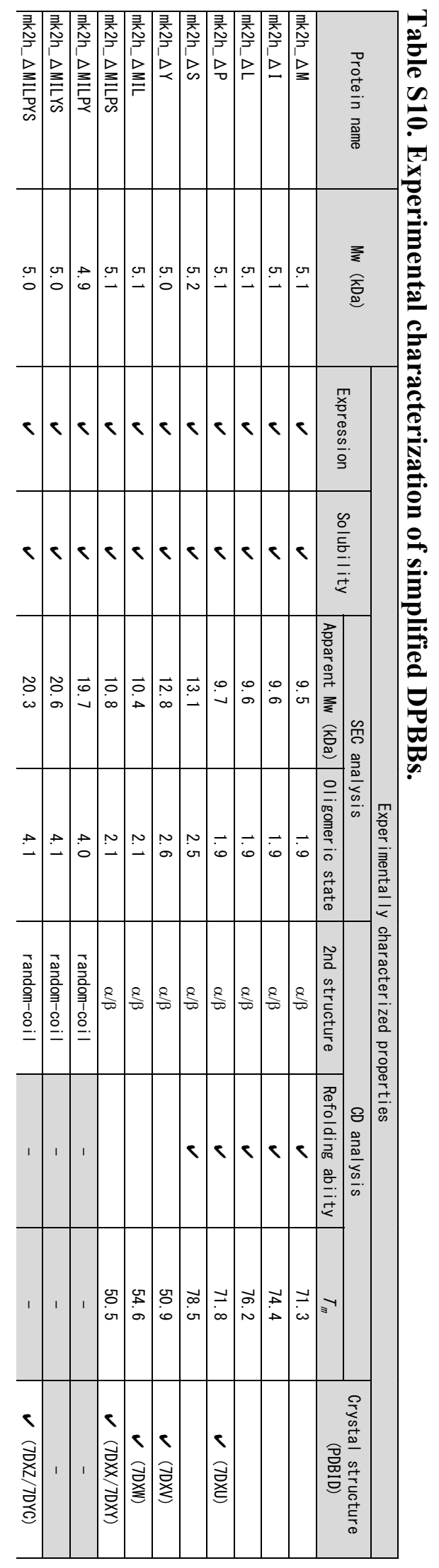


bioRxiv preprint doi: https://doi.org/10.1101/2021.02.22.432383; this version posted February 23, 2021. The copyright holder for this

preprint (which was not certified by peer review) is the author/funder. All rights reserved. No reuse allowed without permission.

Table S11. Gene and primer sequences.

\begin{tabular}{|c|c|}
\hline Protein name & Sequence \\
\hline \multicolumn{2}{|c|}{ Isolated DPBBs from $V C P$ proteins } \\
\hline tavCP_DPBB & $\begin{array}{l}\text { AAGTCCTCITTCAGGGACCCATGGAAAGCAACAACGGTATTATTCTGGGTGTTGCAGAAGCAAATAGCACCGATCCGGGTATGAGCCGTGTTCGTCTGGATGA } \\
\text { AAGCAGCCGTCGTCTGCTGGATGCAGAAATGGTGATGTGTGGAATTGAGAAAGGGGTAAAACGGTTGGTGGTGTTTATCGTGCACGTCGGGAGATGAa } \\
\text { AATAAAGGTATTGTTCGTATCGATAGCGTGATGGGTAATAATTGTGGTGCAAGCATTGGCGATAAAGTGAAGTTCGTAAAGTGGGCTAAGGATCGGAATTCT } \\
\text { GTACAGG }\end{array}$ \\
\hline mkVCP_DPBB & $\begin{array}{l}\text { AAGTCCTCTTTCAGGGACCCATGCCTGGTCTGCCGATTAAACTGGGTGTTGAAAAAGCATATCCGGAAGATGTTGGTAAACGTGCAGTTCGTATGGATAAAGC } \\
\text { AAGCCGTGATCGTATTGGTGTTAGCGAAGGTGATCTGGTGAAAATTACCGGTAGCAAACCACGGTTGCACGTGTTCTGCCTGCAAAAAAGAAGATGTAGGC } \\
\text { AAAGGTATTGTGCGCATGGATAAATATGAAGGTCAGATGCCGGTGCAGCGTTGGTGACCGGTTGAAGTTGATCGTGCAGAATAAGGATCCGAATTCTGTA } \\
\text { CAGG }\end{array}$ \\
\hline apVCP_DPBB & $\begin{array}{l}\text { AAGTCCTCTTTCAGGGACCCATGGCAAATAGCAGGGTTGAACTGGGTGTTAGGGAAGCATATCCGCGTGATGTTGGTCGTAAAATTGTTCGTATTGATCGTCA } \\
\text { GACCGCAGCACGTCTGGGTGTTGAAGTTGGTGATTTTGTAAAGTGAGCAAAGGTGATCGTAGGGTTGTTGCAGTTGTTTGGCCTCTGGGTCGGGATGATGAa } \\
\text { GGTCGTGGTATTATTCGTATGGATGGTTATCTGGGTGCAGCACTGGGTGTTACGGTGGGTGATACCGTTACCGTTGAAAAGCAGATAAGGATCGGAATTCT } \\
\text { GTACAGG }\end{array}$ \\
\hline \multicolumn{2}{|c|}{ Symmetric DPBBs designed using Symmetric-conservation method (SC-design) } \\
\hline apDPBB_sym_63 & 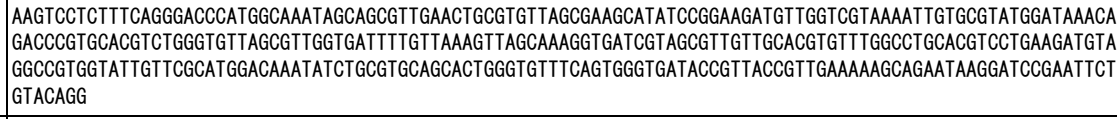 \\
\hline apDPBB_sym_79 & $\begin{array}{l}\text { AAGTCCTCTTTCAGGGACCCATGGCAAATAGCAGCGTTGAACTGGGTGTTCTGGAAGCACGTCCGGAAGATGTTGGTCGTAAAATTGTTCGTATGGATAAACA } \\
\text { GACCCGTGCACGTCTGGGTGTTAGCGTTGGTGATTATGTTGAGTGAAAAAAGTGATCGTAGGGTGGTGCACGTGTTCTGCCTGGGGGTCCTGAGGATGTT } \\
\text { GGGCGGGGTATTGTGGGCATGGACAAATATCTGGGTGAGCACTGGGTGTTTCAGGGGGGATTACGTGGAAGTTAAAAGGTGGATAAGGATCGGATTCT } \\
\text { GTACAGG }\end{array}$ \\
\hline apDPBB_sym_84 & $\begin{array}{l}\text { AAGTCCTCTTTCAGGGACCCATGGCAAATAGCAGGGTTGAACTGGGTGTTGGCGAAGCATATCCGGAAGATGTTGGTCGTAAAATTGTGGGTATGGATAAACA } \\
\text { GACCCGTGCAAACTGGGTGTTAGCGTTGGTGATTATGTGGAAGTTAAAAAAGTTGATCGTAGGGTTGTTGAGGTGTGGGGAAGCCTATCCTGAGGATGTA } \\
\text { GGCCGTGGTATTGTTCGCATGGACAAATATCTGGGTGCCAAATTAGGTGTTTCAGTGGGGGATTATGTTGAGGTGAAAAGGTGGATAAGGATCGGAATTCT } \\
\text { GTACAGG }\end{array}$ \\
\hline mkDPBB_sym_67 & $\begin{array}{l}\text { AAGTCCTCTTTCAGGGACCCATGCCTGGTCTGAGGGTTAAACTGGGTGTTGAAAAAGCATATCCGGAAGATGTTGGTAAACGTATTGTGGGTATGGATAAAGC } \\
\text { AAGCCGTGCACGTCTGGGTGTTAGCGTTGGTGATCTGGTTAAAGTTACCAAAAGCAAAGCGTTGTTGCACGTGTTCTGCCTGCAAAACCTGAAGATGTAGGC } \\
\text { AAAGGCATTGTTCGCATGGACAAATATGAACGTGCAAATCTGGGTGTGTCAGTGGGTGATCCGGTTGAAGTAGATAAGCAGAATAAGGATCGAATTCTGTA } \\
\text { CAGG }\end{array}$ \\
\hline mkDPBB_sym_81 & 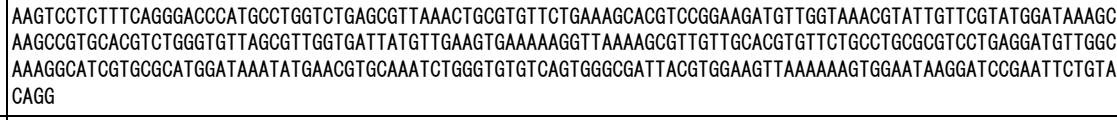 \\
\hline mkDPBB_sym_86 & $\begin{array}{l}\text { AAGTCCTCTTTCAGGGACCCATGCCTGGTCTGAGGGTTAAACTGGGTGTTGGCGAAGCATATCCGGAAGATGTTGGTAAACGTATTGTGGGTATGGATAAAGC } \\
\text { AAGCCGTGCAAACTGGGTGTTAGCGTTGGTGATTATGTGGAGGTAAAAAAGTTAAAGCGTTGTTGCACGTGTGGGGGAGCCTATCCTGAGGATGTAGGC } \\
\text { AAAGGCATTGTTCGCATGGACAAATATGAAGGTGCCAAATTAGGTGTTAGTGTGGGGGATTACGTTGAGGTTAAAAAGTGGAATAAGGATCGGATTCTGTA } \\
\text { CAGG }\end{array}$ \\
\hline mkDPBB_sym1 & $\begin{array}{l}\text { AAGTCCTCTTTCAGGGACCCATGCCTGGTCTGAGGGTTAAACTGGGTGTTGGCGAAGCATATCCGGAAGATGTTGGTAAAGGTATTGTGGGTATGGATAAAGC } \\
\text { AAGCCGTGCAAACTGGGTGTTAGCGTTGGTGATTATGTGGAAGTTAAAAAAGTTCTGTAGTGAAACTGGGCGTGGCAGAGGCCTATCCTGAGGATGTAGGC } \\
\text { AAAGGCATCGTTCGCATGGACAAGGCATCACGTGCCAAATTAGGTGTTCAGTGGGGATTATGTTGAGGTGAAAAAGGTGTAAGGATCGAATTCTGTACAG } \\
\text { G }\end{array}$ \\
\hline mkDPBB_sym2 & 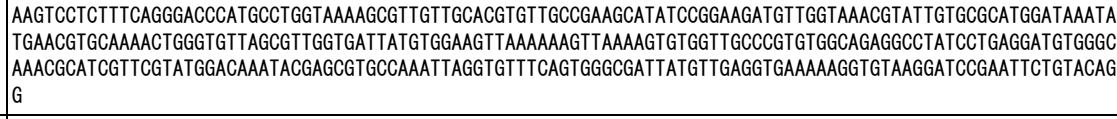 \\
\hline apDPBB_sym1 & 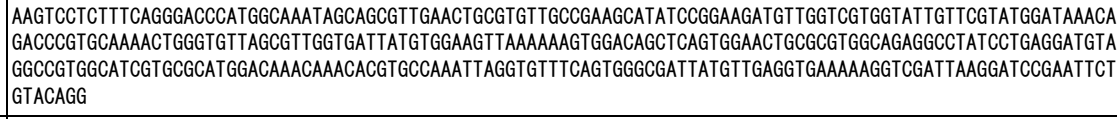 \\
\hline apDPBB_sym2 & 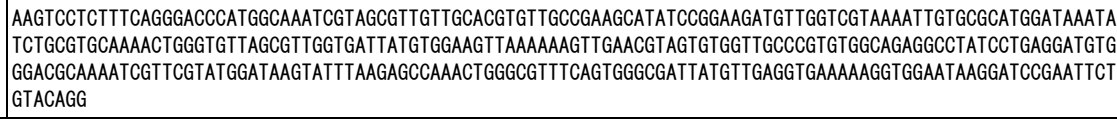 \\
\hline \multicolumn{2}{|c|}{ Symmetric DPBBs designed using reverse evolution engineering method (RE-design) } \\
\hline reDPBB_sym1 & $\begin{array}{l}\text { AAGTCCTCTTTCAGGGACCCCCGATTAAACTGCGTGTTATGGAAGCATATCCGGAaGATGTTGGTAAAGGTATTGTGGGTATGGATAAAGCAAGCCGTGATAA } \\
\text { ACTGGGTGTTTCAGCCGGTGATCTGGTTGAAATTAAAGGTAGCAAAACCCCTATCAAACTTCGCGTGATGGAAGGGTACCCTGAGGATGTAGGCAAAGGCATC } \\
\text { GTTCGCATGGACAAAGCCTCACGTGATAAATTAGGTGGGAGGGCAGGCGACCTGGTGGAATCAAAGGCTAAGGATCCGAATTCTGTACAGG }\end{array}$ \\
\hline reDPBB_sym2 & $\begin{array}{l}\text { AAGTCCTCTTTCAGGGACCCCCGATGAAACTGCGTGTTATGGAAGCATATCCGGAAGATGTTGGTAAAGGTATTGTGGGTATGGATAAAGCAAGCGGTGAAA } \\
\text { ACTGGGTGTTAGTGCCGGTGATCTGGTTGAAATTAAAGGTAGCAAAACCCCGATGAAATTAAGAGTGATGGAAGGGTACCCTGAGGATGTAGGCAAAGGCATC } \\
\text { GTTCGCATGGACAAAGCATCACGGGAGAAACTGGGCGTTTCAGCAGGCGACCTGGTGGAATCAAAGGCTAAGGATCGGAATTCTGTACAGG }\end{array}$ \\
\hline reDPBB_sym3 & $\begin{array}{l}\text { AAGTCCTCTTTCAGGGACCCACCATTAAACTGCGTGTTATGGAAGCATATCCGGAAGATGTTGGTAAAGGTATTGTGGGTATGGATAAAGCAAGCGGTGATAA } \\
\text { AATTGGTGTTAGTGCCGGTGATCTGGTGGAAATTAAAGGTAGAAAACCACAATCAAACTTCGCGTGATGGAAGGGTACCCTGAGGATGTAGGCAAAGGCATC } \\
\text { GTTCGCATGGACAAAGCCTCACGCGATAAAATCGGCGTTTCAGCAGGCGATTIAGTTGAATCAAAGGCTAAGGATCGGAATTCTGTACAGG }\end{array}$ \\
\hline
\end{tabular}


bioRxiv preprint doi: https://doi.org/10.1101/2021.02.22.432383; this version posted February 23, 2021. The copyright holder for this preprint (which was not certified by peer review) is the author/funder. All rights reserved. No reuse allowed without permission.

\begin{tabular}{|c|c|}
\hline reDPBB_sym4 & $\begin{array}{l}\text { AAGTCCTCTTTCAGGGACCCATGCCTGGTAAAAGGGTTGTTGCACGTGTTGCACCGGCACATCCGGAAGATGTTGGTAAAGGTATTGTTCGCATGGATAAATA } \\
\text { TGAACGCCAGAATCTGGGTGTTAGCGTTGGTGATTATGTGGAAGTTAAAAAGCCAAATAGTGGTTGCTCGCGTTGCCCCTGCGCACCCTGAAGATGTAGGC } \\
\text { AAAGGCATCGTGAGAATGGACAAATACGAGGGTCAGAACCTGGGGGTTCAGTGGGGGATTATGTTGAGGTGAAAAAGGCATAAGGATCCGAATTCTGTACAG } \\
\text { G }\end{array}$ \\
\hline reDPBB_sym5 & $\begin{array}{l}\text { AAGTCCTCTTTCAGGGACCCATGCCTGGTAAAAGGGTTGTTGCACGTGTTGCACCGGCATATCCGGAAGATGTTGGTAAAGGTATTGTGGGCATGGATAAATA } \\
\text { TGAACGTGCAAATCTGGGGTTAGCGTTGGTGATTATGTTGAAGTGATAAAGCCAAATAGTGGTTGCTCGCGTTGCCCCTGCCTATCCTGAGGATGTAGGC } \\
\text { AAAGGCATCGTTCGTATGGACAAATACGAGGGTGCCAACCTGGGGGTTCAGTGGGCGATTACGTGGAGTGGACAAAGCATAAGGATCCGAATTCTGTACAG } \\
\text { G }\end{array}$ \\
\hline reDPBB_sym6 & $\begin{array}{l}\text { AAGTCCTCTTTCAGGGACCCATGCCTGGTAAAACGGTTGTTGCACGTGTTCTGCCTGCATATCCGGAAGATGTTGGTAAAGGTATTGTGCGCATGGATAAATA } \\
\text { TGAACGTGCAAAACTGGGGTTAGCGTTGGTGATTATGTTGAAGTTGAAAAGCCAAACCGTGGTGGCTCGCGTGCTGCCAGCCTATCCTGAGGATGTAGGC } \\
\text { AAAGGCATCGTTCGTATGGACAAATACGAGGGTGCCAAATTAGGTGTTTCAGTGGGGGATTACGTGGAGGGGAAAGGCATAAGGATCCGAATTCTGTACAG } \\
\text { G }\end{array}$ \\
\hline reDPBB_sym7 & $\begin{array}{l}\text { AAGTCCTCTTTCAGGGACCCATGCCTGGTAAAAGGGTTGTTGCACGTGTTGCACCGGCATATCCGGAAGATGTTGGTAAAGGTATTGTGCGCATGGATAAATA } \\
\text { TGAACGTGCAAAACTGGGGGTTAGCGTTGGTGATTATGTTGAAGTTGAAAAGCCAAATCAGTGGTTGCTCGCGTTGCCCTGCCTATCCTGAGGATGTAGGC } \\
\text { AAAGGCATCGTTCGTATGGACAAATACGAGGGTGCCAAATTAGGTGTTTCAGTGGGCGATTACGTGGAGTGGAAAGGCATAAGGATCCGAATTCTGTACAG } \\
\text { G }\end{array}$ \\
\hline \multicolumn{2}{|c|}{ Symmetric DPBBs designed using multi-state design method (MS-design) } \\
\hline msDPBB_sym1 & $\begin{array}{l}\text { AAGTCCTCTITCAGGGACCCAGCAGCGTTGTTGCACGTGTTGCACTGGCACATGAAGATGATGTTGGTAAAAACATTGTGGGCATGGATGAAGATCTGATGCG } \\
\text { TAAACTGGGTGTTAAAGTGGTGATTAGGTGGAATCATGAAGAAAGCTCAGTGGTTGCCGGTGTGGCTTAGCGCACGAGGATGATGTGGGCAAAAATATC } \\
\text { GTTCGTATGGACGAGATTTAATGAGAAAACTGGGGGTGAAAGTGGGCGACTATGTTGAAATTATGAAAAAATGGGATCCGAATTCTGTACAGG }\end{array}$ \\
\hline msDPBB_sym2 & $\begin{array}{l}\text { AAGTCCTCTITCAGGGACCCAGCAGCGTTATTGCAGGTGTTGCACTGGCACATGAAGATGATGTTGGTAAAAACATTGTGGGCATGGATGAAGAACTGATGCG } \\
\text { TCTGCTGGGTGTTAAAGTGGTGATCTGGTTGAATCATGAAAGTGAGCAGTGTGATTGCCCGTGTGGCCTTAGCGCACGAGGATGATGTGGGCAAAAATATC } \\
\text { GTTCGTATGGACGAAGATTAATGCGCCTGTTAGGCGTGAAGGTGGGGACCTGGTGGAATTATGAGGGTTAAGGATCCGAATTCTGTACAGG }\end{array}$ \\
\hline \multicolumn{2}{|c|}{ Simplified mk2h } \\
\hline$m k 2 h \_\Delta M$ & 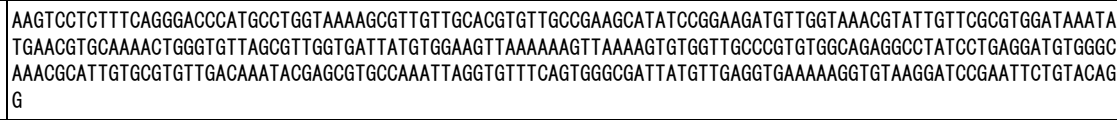 \\
\hline mk2h_ $\Delta \mathrm{I}$ & $\begin{array}{l}\text { AAGTCCTCTTTCAGGGACCCATGCCTGGTAAAAGGGTTGTTGCACGTGTTGGCGAAGCATATCCGGAAGATGTTGGTAAAGGTGTTGTTCGCATGGATAAATA } \\
\text { TGAACGTGCAAAACTGGGTGTTAGCGTTGGTGATTATGTGGAAGTTAAAAAGTTAAAATGTGGTTGCCGGTGTGGCAGAGGCCTATCCTGAGGATGTGGGC } \\
\text { AAACGCGTGGTGCGTATGGACAAATACGAGGGTGCCAAATTAGGTGTTTCAGTGGGGGATTATGTTGAGGTGAAAAGGTGTAAGGATCGAATTCTGTACAG } \\
\text { G }\end{array}$ \\
\hline $\mathrm{mk} 2 \mathrm{~h} \_\Delta \mathrm{L}$ & 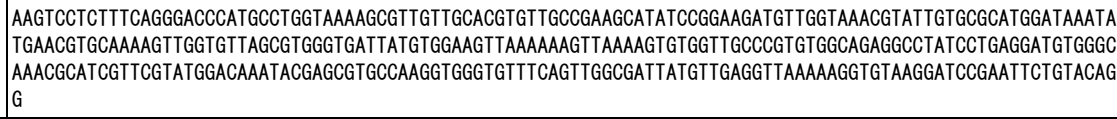 \\
\hline$m k 2 h_{-} \Delta \mathrm{P}$ & $\begin{array}{l}\text { AAGTCCTCTTTCAGGGACCCATGCCTGGTAAAAGGGTTGTTGCACGTGTTGGCGAAGCATATGCAGAAGATGTTGGTAAAGGTATTGTGGGCATGGATAAATA } \\
\text { TGAACGTGCAAAACTGGGGTTAGCGTTGGTGATTATGTGGAAGTTAAAAAGTTAAAATGTGGTTGCCGGTGTGGCAGAGGCCTATGCCGAGGATGTGGGC } \\
\text { AAACGCATCGTTCGTATGGACAAATACGAGGGTGCCAAATTAGGTGTTTCAGTGGGGGATTATGTTGAGGTGAAAAAGGTGTAAGGATCGGAATTCTGTACAG } \\
\text { G }\end{array}$ \\
\hline$m k 2 h \_\Delta S$ & $\begin{array}{l}\text { AAGTCCTCTTTCAGGGACCCATGCCTGGTAAAAAAGTTGTTGCACGTGTTGCCGAAGCATATCCGGAAGATGTTGGTAAACGTATTGTGCGCATGGATAAATA } \\
\text { TGAACGTGCAAAACTGGGGGTGAAGTGGGTGATTATGTTGAAGTGAAAAGGTGAAAAGTGGTGGCACGCGTGGCAGAGGCCTATCCTGAGGATGTGGGC } \\
\text { AAACGCATCGTTCGTATGGACAAATACGAGGGTGCCAAATTAGGGGTTAAAGTTGGGGATTACGTGGAGGCAAAAAGGTGAAGGATCCGAATTCTGTACAG } \\
\text { G }\end{array}$ \\
\hline $\mathrm{mk} 2 \mathrm{~h} \_\Delta \mathrm{Y}$ & 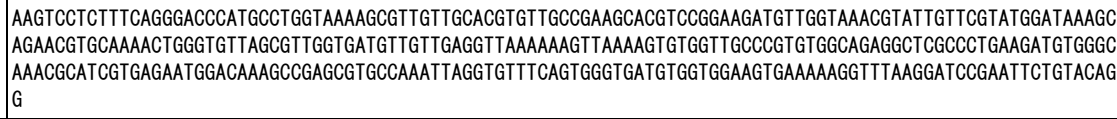 \\
\hline mk2h_ $\Delta \mathrm{MIL}$ & 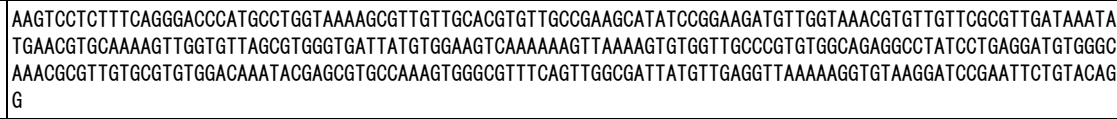 \\
\hline mk2h_AMILPS & $\begin{array}{l}\text { AAGTCCTCTTTCAGGGACCCATGCCTGGTAAAAAAGTTGTTGCACGTGTTGCCGAAGCATATGCCGAAGATGTTGGTAAACGTGTTGTTCGGGTTGATAAATA } \\
\text { TGAACGTGCAAAAGTTGGGTGAAGTGGGTGATTATGTGGAAGTGAAAAGGTGAAAAGTGGTGGCACGCGTGGCAGAGGCGTATGCAGAGGATGTGGG } \\
\text { AAACGCGTTGTGCGTGTGGACAAATACGAGGGTGCCAAAGTGGGGGTTAAAGTTGGGGATTATGTTGAGGTTAAGAAGGTGAAGGATCCGAATTCTGTACAG } \\
\text { G }\end{array}$ \\
\hline mk2h_ $\triangle M I L P Y$ & $\begin{array}{l}\text { AAGTCCTCTTTCAGGGACCCATGCCTGGTAAAAGGGTTGTTGCACGTGTTGGCGAAGCACGTGCCGAAGATGTTGGTAAAGGTGTTGTTCGTGTTGATAAAGC } \\
\text { AGAACGTGCAAAGTTGGTGTTAGCGTTGGTGATGTTGTGGAAGTGAAAAGGTGAATCAGTGGTTGCCGGTGTGGCAGAGGCACGCGCAGAGGATGTGGGC } \\
\text { AAACGTGTGGTGCGCGTGGACAAGGCCGAGGGTGCCAAAGTGGGGGTTTCAGTGGGTGATGTGGTTGAGGTTAAAAAGGTGTAAGGATCCGAATTCTGTACAG } \\
\text { G }\end{array}$ \\
\hline mk2h_LMILYS & $\begin{array}{l}\text { AAGTCCTCTTTCAGGGACCCATGCCTGGTAAAAAAGTTGTTGCACGTGTTGGCGAAGCACGTCCGGAAGATGTTGGTAAACGTGTTGTTCGTGTTGATAAAGC } \\
\text { AGAACGTGCAAAGTTGGTGTTAAAGTGGGTGATGTTGTGGAAGTGAAAAGGTGAAAAGTGGTGGCACGCGTGGCAGAGGCTCGCCCTGAAGATGTGGGC } \\
\text { AAACGCGTGGTGCGCGTGGATAAAGCCGAGGGTGCCAAGGTGGGGGTGAAAGTTGGAGATGTGGTTGAAGTTAAAAAAGTGTAAGGATCGAATTCTGTACAG } \\
\text { G }\end{array}$ \\
\hline mk2h_SMILPYS & $\begin{array}{l}\text { AAGTCCTCTTTCAGGGACCCATGCCTGGTAAAAAAGTTGTTGCACGTGTTGCCGAAGCACGTGCCGAAGATGTTGGTAAACGTGTTGTTCGTGTTGATAAAGC } \\
\text { AGAACGTGCAAAAGTTGGGTTAAAGTGGGTGATGTTGTGGAAGTGAAAAGGTGAAAAAGTGGTGGCACGCGTGGCAGAGGCTGGTGAGAGGATGTGGGC } \\
\text { AAACGGGTGGTGCGCGTGGATAAAGCCGAGGGTGCCAAGGTGGGGGTGAAAGTTGGAGATGTGGTTGAAGTTAAAAAAGTGTAAGGATCCGAATTCTGTACAG } \\
\text { G }\end{array}$ \\
\hline
\end{tabular}


bioRxiv preprint doi: https://doi.org/10.1101/2021.02.22.432383; this version posted February 23, 2021. The copyright holder for this preprint (which was not certified by peer review) is the author/funder. All rights reserved. No reuse allowed without permission.

\begin{tabular}{|c|c|}
\hline Primers & \\
\hline Cloning_upstream & AAGTCCTCTTTCAGGGACCC \\
\hline Cloning_downstream & CCTGTACAGAATTCGGATCC \\
\hline mk1/2_half & CCTGTACAGAATTCGGATCCTTAAACTTITTTAACTTCCAC \\
\hline ap1_half & CCTGTACAGAATTCGGATCCTTAGTCCACTTTTTTAACTTCCAC \\
\hline ap2_half & CCTGTACAGAATTCGGATCCTTATTCAACTTTTTTAACTTCCAC \\
\hline mk2_ $\Delta$ MIL_half & CCTGTACAGAATTCGGATCCTTAAACTTTTTTGACTTCCACATAATCAC \\
\hline mk2_ $\Delta Y$ Y half & CCTGTACAGAATTCGGATCCTTAAACTTTTTTAACCTCAACAAC \\
\hline mk2_ $\Delta S_{\text {Shhalf }}$ & CCTGTACAGAATTCGGATCCTTACACCTTTTTCACTTCAACA \\
\hline mk2_ $\Delta \mathrm{P} / \mathrm{M} / \mathrm{I} / \mathrm{L}$ _half & СCTGTACAGAATTCGGATCCTTAAACTTTTTTAACTTCCACATAATCAC \\
\hline mk2_AMILPS/MILPY/MILYS/MILPYS_half & CCTGTACAGAATTCGGATCCTTACACCTTTTTCACTTCCAC \\
\hline
\end{tabular}




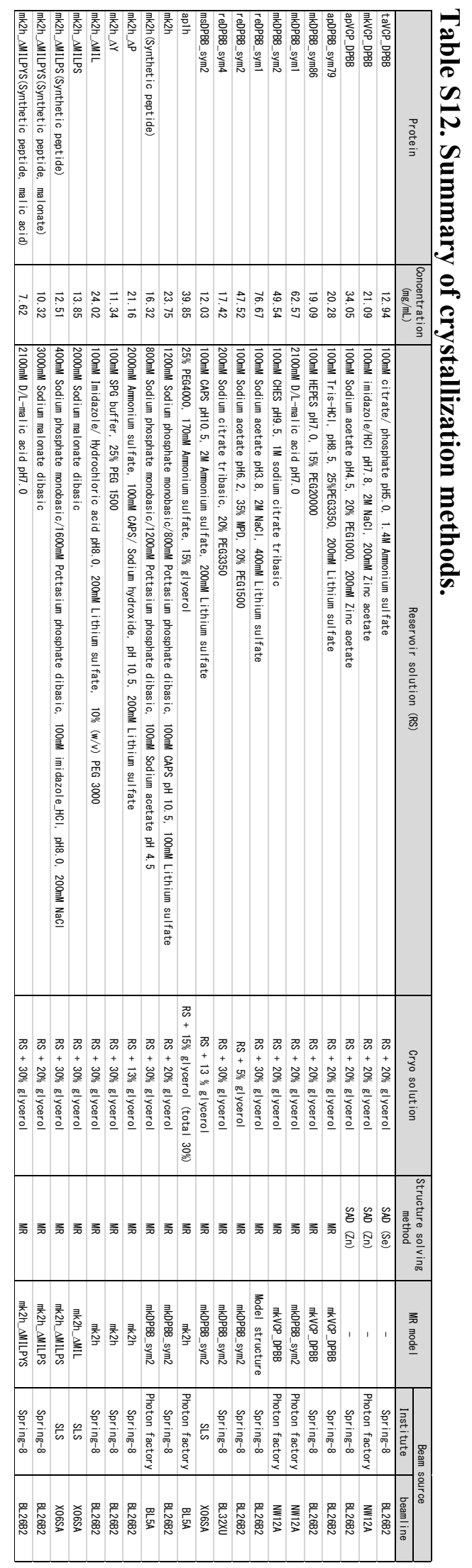




\section{References}

1. R. Furukawa, M. Nakagawa, T. Kuroyanagi, S. I. Yokobori, A. Yamagishi, Quest for Ancestors of Eukaryal Cells Based on Phylogenetic Analyses of Aminoacyl-tRNA Synthetases. J. Mol. Evol. 84, 51-66 (2017).

2. S. F. Altschul, T. L. Madden, A. A. Schäffer, J. Zhang, Z. Zhang,,W. Miller, D. J. Lipman, Gapped BLAST and PSI-BLAST: a new generation of protein database search programs. Nucleic Acids Res. 25, 3389-3402 (1997).

3. A. R. D. Voet, H. Noguchi, C. Addy, D. Simoncini, D. Terada, S. Unzai, S. Y. Park, K. Y. J. Zhang, J. R. H. Tame, Computational design of a self-assembling symmetrical $\beta$ propeller protein. Proc. Natl. Acad. Sci. U. S. A. 111, 15102-15107 (2014).

4. H. Noguchi, C. Addy, D. Simoncini, S. Wouters, B. Mylemans, L. Van Meervelt, T. Schiex, K. Y. J. Zhang, J. R. H. Tame, A. R. D. Voet, Computational design of symmetrical eight-bladed $\beta$-propeller proteins. IUCrJ. 6, 46-55 (2019).

5. D. Terada, A. R. D. Voet, H. Noguchi, K. Kamata, M. Ohki, C. Addy, Y. Fujii, D. Yamamoto, Y. Ozeki, J. R. H. Tame, K. Y. J. Zhang, Computational design of a symmetrical $\beta$-trefoil lectin with cancer cell binding activity. Sci. Rep. 7, 1-13 (2017).

6. S. Whelan, N. Goldman, A general empirical model of protein evolution derived from multiple protein families using a maximum-likelihood approach. Mol. Biol. Evol. 18, 691699 (2001).

7. D. T. Jones, W. R. Taylor, J. M. Thornton, The rapid generation of mutation data matrices from protein sequences. Bioinformatics. 8, 275-282 (1992).

8. H. Ashkenazy, O. Penn, A. Doron-Faigenboim, O. Cohen, G. Cannarozzi, O. Zomer, T. Pupko, FastML: A web server for probabilistic reconstruction of ancestral sequences. Nucleic Acids Res. 40, 580-584 (2012).

9. A. R. D. Voet, D. Simoncini, J. R. H. Tame, K. Y. J. Zhang, Evolution-inspired computational design of symmetric proteins. Methods Mol. Biol. 1529, 309-322 (2017).

10. F. DiMaio, A. Leaver-Fay, P. Bradley, D. Baker, I. André, Modeling symmetric macromolecular structures in Rosetta3. PLoS One. 6 (2011), doi:10.1371/journal.pone.0020450.

11. S. Chaudhury, S. Lyskov, J. J. Gray, PyRosetta: A script-based interface for implementing molecular modeling algorithms using Rosetta. Bioinformatics. 26, 689-691 (2010).

12. J. Vucinic, D. Simoncini, M. Ruffini, S. Barbe, T. Schiex, Positive multistate protein design. Bioinformatics. 36, 122-130 (2020).

13. F. Berenger, R. Shrestha, Y. Zhou, D. Simoncini, K. Y. J. Zhang, Durandal: Fast exact clustering of protein decoys. J. Comput. Chem. 33, 471-474 (2012).

14. R. M. Kramer, V. R. Shende, N. Motl, C. N. Pace, J. M. Scholtz, Toward a molecular understanding of protein solubility: Increased negative surface charge correlates with increased solubility. Biophys. J. 102, 1907-1915 (2012).

15. S. Jones, J. M. Thornton, Review Principles of protein-protein interactions. Proc. Natl. Acad. Sci. U. S. A. 93, 13-20 (1996).

16. J. Janin, S. Miller, C. Chothia, Surface, subunit interfaces and interior of oligomeric proteins. J. Mol. Biol. 204, 155-164 (1988).

17. F. Chiti, M. Stefani, N. Taddei, G. Ramponi, C. M. Dobson, Rationalization of mutational effects on protein aggregation rates. Nature. 424, 805-808 (2003).

18. D. Simoncini, T. Schiex, K. Y. J. Zhang, Balancing exploration and exploitation in population-based sampling improves fragment-based de novo protein structure prediction. Proteins. 85, 852-858 (2017).

19. J. A. Maier, C. Martinez, K. Kasavajhala, L. Wickstrom, K. E. Hauser, C. Simmerling, ff14SB: Improving the Accuracy of Protein Side Chain and Backbone Parameters from ff99SB. J. Chem. Theory Comput. 11, 3696-3713 (2015).

20. D. A. Case, R. M. Betz, D. S. Cerutti, T. Cheatham, T. Darden, R. E. Duke, T. J. Giese, H. Gohlke, A. W. Götz, N. Homeyer, S. Izadi, P. Janowski, J. Kaus, A. Kovalenko, T. S. Lee, 
S. LeGrand, P. Li, C. Lin, T. Luchko, R. Luo, B. Madej, D. Mermelstein, K. M. Merz, G. Monard, H. Nguyen, H. T. Nguyen, I. Omelyan, A. Onufriev, D. R. Roe, A. Roitberg, C. Sagui, C. L. Simmerling, W. M. Botello-Smith, J. Swails, R. C. Walker, J. Wang, R. M. Wolf, X. Wu, L. Xiao, P. A. Kollman, Amber 16. University of California, San Francisco. (2016).

21. H. J. C. Berendsen, J. P. M. Postma, W. F. van Gunsteren, A. Dinola, J. R. Haak, Molecular dynamics with coupling to an external bath. J. Chem. Phys. 81, 3684-3690 (1984).

22. T. Darden, D. York, L. Pedersen, Particle mesh Ewald: An $N \cdot \log (\mathrm{N})$ method for Ewald sums in large systems. J. Chem. Phys. 98, 10089-10092 (1993).

23. J. P. Ryckaert, G. Ciccotti, H. J. C. Berendsen, Numerical integration of the cartesian equations of motion of a system with constraints: molecular dynamics of n-alkanes. $J$. Comput. Phys. 23, 327-341 (1977).

24. D. R. Roe, T. E. Cheatham, PTRAJ and CPPTRAJ: Software for processing and analysis of molecular dynamics trajectory data. J. Chem. Theory Comput. 9, 3084-3095 (2013).

25. Y. Yamada, N. Matsugaki, L. M. G. Chavas, M. Hiraki, N. Igarashi, S. Wakatsuki, Data management system at the photon factory macromolecular crystallography beamline. $J$. Phys. Conf. Ser. 425 (2013), doi:10.1088/1742-6596/425/1/012017.

26. M. Hiraki, Y. Yamada, L. M. G. Chavas, S. Wakatsuki, N. Matsugaki, Improvement of an automated protein crystal exchange system PAM for high-throughput data collection. $J$. Synchrotron Radiat. 20, 890-893 (2013).

27. G. Ueno, H. Kanda, R. Hirose, K. Ida, T. Kumasaka, M. Yamamoto, RIKEN structural genomics beamlines at the SPring-8; high throughput protein crystallography with automated beamline operation. J. Struct. Funct. Genomics. 7, 15-22 (2006).

28. S. Ito, G. Ueno, M. Yamamoto, DeepCentering: fully automated crystal centering using deep learning for macromolecular crystallography. J. Synchrotron Radiat. 26, 1361-1366 (2019).

29. N. Okazaki, K. Hasegawa, G. Ueno, H. Murakami, T. Kumasaka, M. Yamamoto, Mail-in data collection at SPring-8 protein crystallography beamlines. J. Synchrotron Radiat. 15, 288-291 (2008).

30. H. Murakami, G. Ueno, N. Shimizu, T. Kumasaka, M. Yamamoto, Upgrade of automated sample exchanger SPACE. J. Appl. Crystallogr. 45, 234-238 (2012).

31. K. Hirata, K. Yamashita, G. Ueno, Y. Kawano, K. Hasegawa, T. Kumasaka, M. Yamamoto, Zoo: An automatic data-collection system for high-throughput structure analysis in protein microcrystallography. Acta Crystallogr. Sect. D Struct. Biol. 75, 138150 (2019).

32. Y. Nakamura, S. Baba, N. Mizuno, T. Irie, G. Ueno, K. Hirata, S. Ito, K. Hasegawa, M. Yamamoto, T. Kumasaka, Computer-controlled liquid-nitrogen drizzling device for removing frost from cryopreserved crystals. Acta Crystallogr. Sect. F Struct. Biol. Commun. 76, 616-622 (2020).

33. W. Kabsch, XDS. Acta Crystallogr. D. Biol. Crystallogr. 66, 125-32 (2010).

34. P. D. Adams, P. V. Afonine, G. Bunkóczi, V. B. Chen, I. W. Davis, N. Echols, J. J. Headd, L. W. Hung, G. J. Kapral, R. W. Grosse-Kunstleve, A. J. McCoy, N. W. Moriarty, R. Oeffner, R. J. Read, D. C. Richardson, J. S. Richardson, T. C. Terwilliger, P. H. Zwart, PHENIX: A comprehensive Python-based system for macromolecular structure solution. Acta Crystallogr. Sect. D Biol. Crystallogr. 66, 213-221 (2010).

35. T. C. Terwilliger, P. D. Adams, R. J. Read, A. J. McCoy, N. W. Moriarty, R. W. GrosseKunstleve, P. V. Afonine, P. H. Zwart, L. W. Hung, Decision-making in structure solution using Bayesian estimates of map quality: The PHENIX AutoSol wizard. Acta Crystallogr. Sect. D Biol. Crystallogr. 65, 582-601 (2009).

36. P. Emsley, K. Cowtan, Coot: Model-building tools for molecular graphics. Acta Crystallogr. Sect. D Biol. Crystallogr. 60, 2126-2132 (2004). 
37. H. Xue, K. L. Tong, C. Marck, H. Grosjean, J. T. F. Wong, Transfer RNA paralogs: Evidence for genetic code-amino acid biosynthesis coevolution and an archaeal root of life. Gene. 310, 59-66 (2003).

38. Z. Yu, K. Takai, A. Slesarev, H. Xue, J. T. F. Wong, Search for primitive methanopyrus based on genetic distance between Val- and Ile-tRNA synthetases. J. Mol. Evol. 69, 386394 (2009).

39. J. Nölling, A. Elfner, J. R. Palmer, V. J. Steigerwald, T. D. Pihl, J. A. Lake, J. N. Reeve, Phylogeny of Methanopyrus kandleri based on methyl coenzyme M reductase operons. Int. J. Syst. Bacteriol. 46, 1170-1173 (1996).

40. S. Akanuma, Y. Nakajima, S. I. Yokobori, M. Kimura, N. Nemoto, T. Mase, K. I. Miyazono, M. Tanokura, A. Yamagishi, Experimental evidence for the thermophilicity of ancestral life. Proc. Natl. Acad. Sci. U. S. A. 110, 11067-11072 (2013).

41. M. N. Nguyen, K. P. Tan, M. S. Madhusudhan, CLICK - Topology-independent comparison of biomolecular 3D structures. Nucleic Acids Res. 39, 24-28 (2011).

42. M. N. Nguyen, M. S. Madhusudhan, Biological insights from topology independent comparison of protein 3D structures. Nucleic Acids Res. 39 (2011), doi:10.1093/nar/gkr348.

43. R. M. Castillo, K. Mizuguchi, V. Dhanaraj, A. Albert, T. L. Blundell, A. G. Murzin, A six-stranded double-psi $\beta$ barrel is shared by several protein superfamilies. Structure. 7, 227-236 (1999).

44. Z. F. Burton, K. Opron, G. Wei, J. H. Geiger, A model for genesis of transcription systems. Transcription. 7, 1-13 (2016). 